\title{
Angiospermas nativas documentadas en la literatura para el Estado de México, México
}

\section{Native angiosperms documented in the literature for the State of Mexico, Mexico}

\author{
Isabel Martínez-De La Cruz' (D), José Luis Villaseñor² (D), Luis Isaac Aguilera Gómez³ (D), Martín Rubí Arriaga 4,5 (D)
}

1 Universidad Autónoma del Estado de México, Programa de Doctorado en Ciencias Agropecuarias y Recursos Naturales, Facultad de Ciencias Agrícolas, Campus Universitario "El Cerrillo", El Cerrillo Piedras Blancas, km 15.5 carretera Toluca-Ixtlahuaca, 50200 Toluca, Estado de México, México.

2 Universidad Nacional Autónoma de México, Instituto de Biología, Departamento de Botánica, 04510 Cd. Mx. México.

3 Universidad Autónoma del Estado de México, Facultad de Ciencias, Campus Universitario "El Cerrillo", El Cerrillo Piedras Blancas, km 15.5 carretera Toluca-Ixtlahuaca, 50200 Toluca, Estado de México, México.

4 Universidad Autónoma del Estado de México, Facultad de Ciencias Agrícolas, Centro de Investigación y Estudios Avanzados en Fitomejoramiento (CIEAF), Campus Universitario "El Cerrillo", El Cerrillo Piedras Blancas, km 15.5 carretera TolucaIxtlahuaca, 50200 Toluca, Estado de México, México.

5 Autor para la correspondencia: m_rubi65@yahoo.com.mx

Recibido: 31 de julio de 2017 Revisado: 30 de agosto de 2017

Aceptado: 12 de febrero de 2018

Primero en línea: 29 de mayo de 2018.

Publicado: 1 de julio de 2018.

Citar como

Martínez-De La Cruz, I., J. L. Villaseñor, L. I. Aguilera Gómez y M. Rubí Arriaga. 2018. Angiospermas nativas documentadas en la literatura para el Estado de México, México. Acta Botanica Mexicana 124: 135-217. DOI: 10.21829/ abml24.2018.1273

$\mathrm{DOI}$

10.21829/abml24.2018.1273

\section{Resumen:}

Antecedentes y Objetivos: En 1979 se publicó por primera vez la riqueza de plantas vasculares en el Estado de México. La información allí presentada ha perdido actualidad debido al incremento de aportaciones florísticas. El objetivo de este trabajo es contribuir al conocimiento de la flora del Estado de México, a partir de la literatura florística-taxonómica disponible.

Métodos: Se integró una base de datos con la información proveniente de 351 documentos. Se elaboró un mapa que ilustra la ubicación geográfica de los inventarios florísticos y se determinó, mediante el índice de Sørensen, la similitud florística entre inventarios.

Resultados clave: Se compiló una lista con 169 familias, 1103 géneros y 3924 especies de angiospermas. Asteraceae (153 géneros y 588 especies), Fabaceae ( 82 y 372), Poaceae ( 85 y 340) y Orchidaceae (70 y 231) son las familias mejor representadas. Muhlenbergia (54 especies), Salvia (53) e Ipomoea (51) presentaron el mayor número de especies. Las tesis fundamentan $80 \%$ de los taxa. El índice de similitud reveló que la mayoría de las especies están restringidas a una o dos compilaciones. La distribución de los sitios de exploración botánica no es uniforme, se han realizado más trabajos florísticos en las zonas sur y suroeste del estado. Las investigaciones se han llevado a cabo principalmente en sitios próximos a vías de comunicación e instituciones educativas de trascendencia y las regiones montañosas han sido pobremente recolectadas.

Conclusiones: La información sobre la riqueza de angiospermas acumulada en la literatura resulta fundamental para determinar la distribución geográfica de las especies en los municipios del estado, adicionar información sobre endemismo, usos actuales y potenciales, así como establecer programas de conservación de los recursos naturales.

Palabras clave: especies endémicas, inventario florístico, plantas con flores, riqueza florística.

\section{ABSTRACT:}

Background and Aims: The richness of vascular plants in the State of Mexico was published for the first time in 1979. Since then, these data have become outdated due to the increasing number of floristic contributions. The objective of this work is contributing to the knowledge of the flora of the State of Mexico, from the available floristic-taxonomic literature.

Methods: A database was integrated with information from 351 publications. A map was drawn illustrating the geographical location of floristic inventories and the floristic similarities between these inventories was determined using the Sørensen index.

Key results: A list of 169 families, 1103 genera and 3924 species of angiosperms was compiled, where Asteraceae (153 genera and 588 species), Fabaceae (82 and 372), Poaceae ( 85 and 340) and Orchidaceae (70 and 231) are the best represented families. Muhlenbergia (54 species), Salvia (53) and Ipomoea (51) had the highest number of species. Unpublished theses account for $80 \%$ of the taxa. The similarity index revealed that most species are restricted to one or two compilations. The distribution of botanical exploration sites is not uniform, more floristic work has been realized in the southern and southwestern areas. Research has been carried out mainly on sites close to roads and educational institutions of importance. The mountainous regions are poorly explored.

Conclusions: The information on angiosperm richness accumulated in the literature is fundamental to determine the geographical distribution of species in the municipalities of the state, to add information on endemism, current and potential uses, as well as to establish conservation programs of its natural resources. Key words: endemic species, floristic inventory, floristic richness, flowering plants. 


\section{INTRODUCCIÓN}

La documentación acerca de la flora del Estado de México se inició de manera formal con los trabajos de campo y publicación de fascículos que Maximino Martínez y Eizi Matuda llevaron a cabo entre los años 1952 y 1957 (Martínez y Matuda, 1979). Sin embargo, muchas familias no fueron tratadas; después de transcurridas más de tres décadas, la información allí presentada ha perdido actualidad y puede considerarse obsoleta, debido a las nuevas aportaciones en la exploración botánica y a los avances en la taxonomía (Ibarra-Manríquez et al., 1995; Villaseñor, 2003, 2016).

A partir de la década de 1980, el conocimiento sobre la flora del estado se incrementó notablemente con trabajos de campo y laboratorio (Apéndices 1, 2). Entre las principales contribuciones que intentan integrar la información florística de la entidad mexiquense se pueden mencionar las siguientes: 1) Galicia-Miranda (1992) revisó las colecciones de Eizi Matuda, 2) Alcántara-Alameda (2006) elaboró un inventario resultado de la consulta de ejemplares botánicos depositados en herbarios nacionales, de la búsqueda electrónica en bases de datos nacionales y extranjeros (Comisión Nacional para el Conocimiento y Uso de la Biodiversidad, The Missouri Botanical Garden, Instituto de Ecología, A.C., The New York Botanical Garden y The Royal Botanical Gardens, Kew) y de la revisión de literatura florística (30 publicaciones), 3) Garduño-Solórzano et al. (2009) efectuaron un recuento de la riqueza florística del estado a través de la consulta de 40 proyectos del Sistema Nacional de Información sobre Biodiversidad (SNIB), así como de inventarios estatales sobre cactáceas, bromeliáceas, leguminosas, orquídeas, gramíneas, cucurbitáceas y encinos, y 4) Villaseñor y Ortiz (2014) señalaron la existencia de 3799 especies de Magnoliophyta, cantidad documentada principalmente con base en ejemplares de herbario y con información registrada en alrededor de 20 referencias bibliográficas; dicha cifra fue actualizada dos años después, incrementando el número en $27.7 \%$, al alcanzar un total de 4852 especies de angiospermas (Villaseñor, 2016).

Después de una revisión exhaustiva de bibliografía relacionada con la riqueza florística estatal, se presenta un catálogo de las angiospermas nativas con el objetivo de contribuir al conocimiento de la flora del Estado de México. Se planteó la estrategia de realizar un análisis crítico de las angiospermas documentadas en la literatura publicada, incluyendo aquella considerada como literatura gris (informes de proyectos, planes de desarrollo y tesis). Esta compilación podrá detectar lagunas de información o discrepancias sobre especies existentes en el territorio mexiquense no reportadas en documentos convencionales y contribuir a un mejor conocimiento de la riqueza de angiospermas en la entidad.

\section{Materiales y Métodos}

\section{Área de estudio}

El Estado de México se ubica en la parte central de la República Mexicana, entre las coordenadas $18^{\circ} 22^{\prime}$ y $20^{\circ} 17^{\prime}$ de latitud Norte y $98^{\circ} 36^{\prime}$ y $100^{\circ} 37^{\prime}$ de longitud Oeste. Cuenta con una superficie territorial de 22,337.58 $\mathrm{km}^{2}$ que representa $1.1 \%$ del territorio nacional; ocupa la $25^{\text {a }}$ posición en extensión geográfica del país (INEGI, 2016a).

Está constituido por 125 municipios, de los cuales 59 integran la Zona Metropolitana del Valle CuautitlánTexcoco, 16 el Valle de Toluca y seis la Zona Metropolitana de Santiago Tianguistenco. Colinda con los siguientes estados: Hidalgo y Querétaro, al norte; Ciudad de México, Puebla y Tlaxcala, al este; Guerrero y Morelos, al sur; y Michoacán, al oeste (Fig. 1) (POEM, 2009, 2016; INEGI, 2016a).

El relieve es bastante irregular y está integrado por mesetas $(1.05 \%$ de la superficie), valles $(6.28 \%)$, llanuras (15.24\%), lomeríos (34.97\%) y principalmente sierras (42.46\%), de las cuales destacan la Sierra Nevada, ubicada en la parte este de la geografía estatal (en el límite con Puebla) que incluye los volcanes Popocatépetl (5419 m de altitud máxima) e Iztaccíhuatl (5220 $\mathrm{m})$, segundo y tercero más altos de México, respectivamente; la Sierra de San Andrés Timilpan, situada al noroeste; la Sierra del Xinantécatl (centro-sur de la entidad) que alberga al volcán Nevado de Toluca (4680 

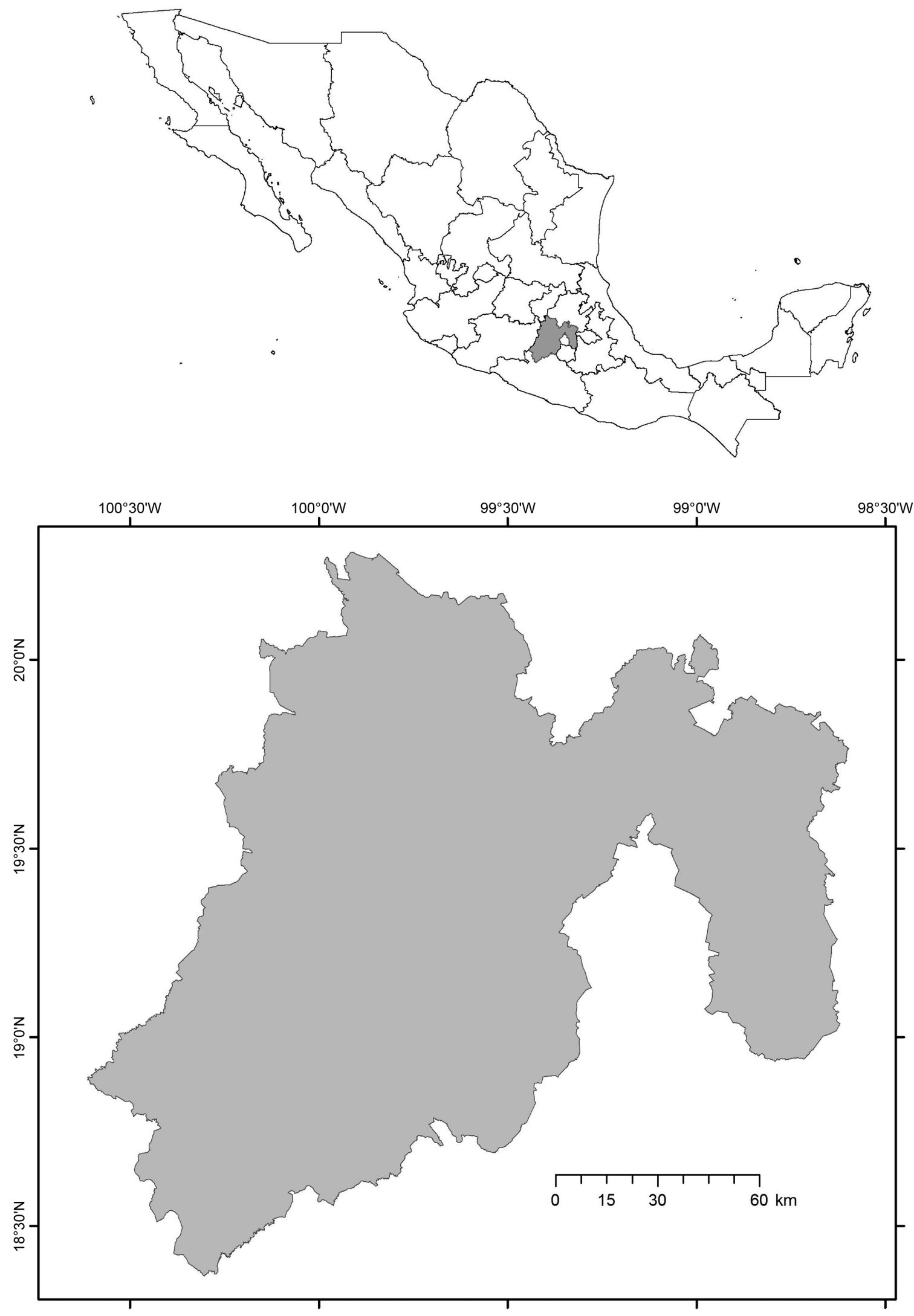

Figura 1: Ubicación geográfica del Estado de México. 
m), el cuarto más alto de México, y las Sierras de Monte Alto y Monte Bajo, que son límites con el oeste de la Ciudad de México (López-Cano et al., 2009; INEGI, 2011, 2016a, b).

Fisiográficamente, $24.4 \%$ de la superficie estatal pertenece a la cuenca del río Balsas y $75.6 \%$ a la Faja Volcánica Transmexicana, en la que se incluyen tres de las cinco elevaciones importantes del país. La altura sobre el nivel del mar va desde $300 \mathrm{~m}$ en los límites con Guerrero, hasta $5419 \mathrm{~m}$ en la cima del Popocatépetl (López-Cano et al., 2009; Neyra-Jáuregui, 2012; INEGI, 2016a, b).

El territorio mexiquense está comprendido dentro de las tres regiones hidrológicas más importantes del país, tanto por su extensión como por el volumen de las corrientes superficiales. La región Pánuco (cubre 35.23\% de la superficie de la entidad) se ubica en la porción noreste y la integran 60 municipios mexiquenses, la región LermaSantiago (25.53\%) se sitúa en la parte centro-noroeste y está conformada por 32 municipios (a este sistema hidrológico pertenece la cuenca alta del río Lerma), y la región del río Balsas (39.24\%) que comprende la parte suroeste y está integrada por 33 municipios. Los cuerpos de agua (29 ríos, dos lagos y ocho presas) abarcan $176.32 \mathrm{~km}^{2} \mathrm{del}$ estado (INEGI, 2001; 2016a).

La variedad de suelos es amplia, asciende a 13 tipos, con predominio de Andosol (4799.1 km²), Feozem $\left(4727.2 \mathrm{~km}^{2}\right)$, Regosol (2656.8 $\left.\mathrm{km}^{2}\right)$, Vertisol (2414.8 $\mathrm{km}^{2}$ ), Cambisol $\left(1960.5 \mathrm{~km}^{2}\right)$ y Leptosol $\left(1749.7 \mathrm{~km}^{2}\right)$ (López-Cano et al., 2009; Sotelo-Ruíz et al., 2011; INEGI, 2016a).

El clima predominante es templado subhúmedo (presente en $61.82 \%$ del territorio estatal); se distribuye particularmente en las inmediaciones de la cuenca de México. En menor grado están los climas cálido subhúmedo y semicálido subhúmedo, ubicados en la región que comprende la cuenca del río Balsas (20.68\%). El clima semifrío subhúmedo está presente en las cercanías de Toluca y en la zona limítrofe con Tlaxcala y Puebla (10.90\%). El clima semifrío húmedo existe en los límites con la Ciudad de México y el estado de Morelos (0.67\%). Una fracción al noreste del estado presenta clima semiseco templado (5.77\%). El clima frío se concentra en las partes altas de los volcanes Nevado de Toluca, Popocatépetl e Iztaccíhuatl $(0.16 \%)$. Además, existe una zona de transición climática (cálido/templado) entre la cuenca del río Balsas y la zona de montaña, en el suroeste del estado (TejeroDíez y Arreguín-Sánchez, 2004; López-Cano et al., 2009; INEGI, 2016a).

Aproximadamente $54.3 \%$ de la superficie del estado es de uso agrícola y $32.4 \%$ es área boscosa, con las siguientes formaciones vegetales: bosque templado (comprende los bosques de Quercus, Pinus, Abies, QuercusPinus, Pinus-Quercus, Pinus-Abies, Abies-Pinus, AbiesQuercus, Quercus-Abies, Alnus y Pinus-Alnus), bosque tropical estacionalmente seco (bosque tropical caducifolio), bosque húmedo de montaña (bosque mesófilo de montaña), bosque tropical húmedo (bosque tropical subcaducifolio) y matorral xerófilo (López-Cano et al., 2009; INEGI, 2016a; Villaseñor, 2016).

\section{Recopilación de la información}

Se realizó la revisión exhaustiva de documentos florístico-taxonómicos que reportan a las angiospermas que prosperan en el Estado de México. La literatura no publicada (gris) se circunscribió a informes de proyectos, planes de desarrollo y tesis depositadas en bibliotecas de instituciones educativas asentadas en el territorio mexiquense, así como en el Valle de México. La publicada incluyó artículos, capítulos de libros y libros. Además, se consideró la Flora Fanerogámica del Valle de México (Calderón de Rzedowski y Rzedowski, 2005), debido a que es una zona que incluye la cuarta parte del territorio mexiquense. También se revisaron los fascículos publicados por proyectos florísticos que se han llevado a cabo en estados adyacentes a la entidad mexiquense, como son los de la Flora del Bajío y de regiones adyacentes (Rzedowski y Calderón de Rzedowski, 1991-2014) y otros de la Flora de Guerrero (Diego-Pérez y Fonseca, 1995-2014), documentos que indican la distribución geográfica de las especies y mencionan cuáles están presentes en el Estado de México. 


\section{Base de datos}

La información recopilada a partir de las referencias bibliográficas permitió integrar una base de datos en el programa Microsoft Access, con los siguientes campos: clase, familia, género, epíteto específico, autor, estatus de la especie en México (endémica o nativa) (Espejo-Serna y López-Ferrari, 2004; Pulido-Esparza et al., 2009; EspejoSerna, 2012; García-Mendoza y Meave, 2012; Villaseñor, 2016) y referencia bibliográfica de cada registro de las especies. En la lista generada de angiospermas del Estado de México, se incluyeron las que se registraron de la revisión bibliográfica y se sabe que cuentan al menos con un ejemplar de herbario recolectado en el territorio, lo que valida su presencia (Villaseñor, 2016). Es importante mencionar que no se consideraron las categorías taxonómicas inferiores al rango de especie.

Para evitar sinonimias, en primera instancia se cotejó la lista de angiospermas recopilada en el presente trabajo con el catálogo de las plantas vasculares nativas de México (Villaseñor, 2016). Para las discrepancias entre estos trabajos se tomaron en cuenta los criterios de especies aceptadas en el catálogo florístico-taxonómico del bosque húmedo de montaña en México (Villaseñor, 2010), así como en las páginas electrónicas World Checklist of Selected Plant Families (WCSP, 2012), Species 2000 \& ITIS Catalogue of Life (Roskov et al., 2016), The Plant List (The Plant List, 2013) y Tropicos (TROPICOS, 2016). Además, se consultaron monografías, revisiones taxonómicas y las Floras del Bajío y de regiones adyacentes, de Guerrero y la Fanerogámica del Valle de México. En caso de conflictos nomenclaturales, se siguió la propuesta del taxónomo especialista o se aplicó el criterio de prioridad nomenclatural.

Las angiospermas se agruparon en Magnoliides, Monocotiledóneas y Eudicotiledóneas, de acuerdo con el sistema de clasificación del Angiosperm Phylogeny Group (APG IV, 2016). Las especies registradas se ordenaron alfabéticamente por familia. Para homogeneizar las abreviaturas de los autores de los nombres científicos se consultó la página electrónica The International Plant Names Index (IPNI, 2016). Es importante mencionar que las especies registradas como introducidas (Espinosa-García, 2000; Villaseñor y Espinosa-García, 2004; Villaseñor et al., 2013) fueron excluidas del inventario.

\section{Elaboración de mapas de distribución geográ- fica de los inventarios}

Para determinar si los lugares de exploración botánica son adyacentes a instituciones educativas, vías de comunicación o en zonas de difícil acceso, se elaboró un mapa que muestra la ubicación geográfica de los inventarios florísticos. Las coordenadas geográficas de los sitios de estudio se obtuvieron a partir de la información disponible en la literatura consultada, a través de la georreferenciación en Google Earth Version 6.2 (Google Inc., 2009), mediante el empleo del programa Mapa Digital de México Versión 6.3.0 (INEGI, 2017) y el Sistema para la Consulta de Información Censal (INEGI, 2013); posteriormente se incorporaron al programa ArcGIS Versión 10.2 (ESRI, 2013). En el caso de que el área correspondiera a un polígono, se procedió a obtener el centroide de la superficie (intersección de los ejes largo y ancho del área muestreada). La ubicación geográfica de las instituciones educativas y de investigación se obtuvo a través del programa Google Earth Version 6.2 (Google Inc., 2009).

\section{Semejanza florística entre los inventarios}

La similitud florística entre los inventarios reportados en la literatura se estimó mediante un análisis de conglomerados con ayuda del Paleontological Statistics Software (PAST) Versión 3.15 (Hammer et al., 2001). Para ello se empleó el índice de similitud de Sørensen-Dice, el cual considera el desequilibrio entre cero y uno (siempre con más ceros que unos) y se recomienda para matrices binarias (Badii et al., 2008; Ruiz-Jiménez et al., 2012). Los valores de semejanza obtenidos permitieron elaborar un dendrograma a través del método UPGMA (método de agrupamiento no ponderado utilizando medias aritméticas, por sus siglas en inglés). Únicamente se tomaron en cuenta los inventarios florísticos puntuales y que presentaron una riqueza superior a 140 especies (se excluyeron los inventarios incluidos en el primer cuartil de riqueza). 
Para comparar la riqueza de especies en áreas de diferentes tamaños, se empleó el índice de biodiversidad taxonómica (Squeo et al., 1998), el cual considera el número de especies, dividido por el logaritmo natural del área en kilómetros cuadrados.

\section{Resultados}

La revisión de literatura florística-taxonómica, plasmada en la base de datos de las angiospermas del Estado de México, permitió obtener una lista preliminar con 5949 especies, resultado de la información registrada en 351 contribuciones (Cuadro 1, Apéndices 1, 2). Mediante la depuración taxonómica se corrigieron errores tipográficos en la escritura de los nombres científicos, los cuales eran considerados como especies diferentes, aun cuando se trataba del mismo taxon. Otras angiospermas estaban citadas tanto con el nombre aceptado como con el sinónimo, lo cual originó una duplicación, misma que sobrestima aproximadamente en un tercio la riqueza de plantas con flores reconocidas para el estado. Se identificaron 1378 sinonimias, pertenecientes a 1114 especies, de las cuales 897 (80\%) registraron un sinónimo y en menor proporción están las que poseen dos $(176,15 \%)$, tres $(37,3 \%)$, cuatro $(2,1 \%)$ y cinco $(2,1 \%)$.

Se excluyeron del análisis 301 angiospermas consideradas introducidas (exóticas) a México. Resulta importante señalar que 346 especies (Apéndice 3) reportadas para el estado por diferentes autores no se incluyeron. Algunas de ellas fueron consideradas determinaciones erróneas, mismas que al validar el nombre no contaron con un ejemplar de herbario que respalde su presencia en el estado (Villaseñor, 2016). Es necesario localizar los ejemplares botánicos de respaldo en las colecciones, verificar su determinación y así corroborar su presencia en la entidad, lo que incrementaría la documentación de angiospermas nativas, así como endemismos.

Finalmente, la lista de angiospermas se integró por 169 familias, 1103 géneros y 3924 especies (Apéndice 4, Cuadro 2), de las cuales 3657 angiospermas están reportadas en la literatura florística-taxonómica para el estado y otras 267 especies están citadas en la bibliografía no estric-
Cuadro 1: Tipo de publicaciones compiladas en la base de datos sobre la flora del Estado de México.

\begin{tabular}{|c|c|}
\hline Publicación & Número \\
\hline Tesis & 80 \\
\hline Colegio de Postgraduados & 11 \\
\hline Instituto Politécnico Nacional & 1 \\
\hline Universidad Autónoma Chapingo & 3 \\
\hline Universidad Autónoma del Estado de México & 23 \\
\hline Universidad Autónoma Metropolitana & 1 \\
\hline Universidad Nacional Autónoma de México & 41 \\
\hline Informes de proyectos de investigación & 3 \\
\hline Revistas científicas & 62 \\
\hline Acta Botanica Mexicana & 18 \\
\hline Anales del Instituto de Biología Serie Botánica & 5 \\
\hline Annals of the Missouri Botanical Garden & 1 \\
\hline Biodiversity and Conservation & 1 \\
\hline $\begin{array}{l}\text { Botanical Sciences (Boletín de la Sociedad Botánica de } \\
\text { México) }\end{array}$ & 12 \\
\hline Brittonia & 1 \\
\hline Cactáceas y Suculentas Mexicanas & 1 \\
\hline Ciencias Agrícolas Informa & 2 \\
\hline Economic Botany & 1 \\
\hline Harvard Papers in Botany & 1 \\
\hline Ibugana Boletín del Instituto de Botánica & 1 \\
\hline Polibotánica & 10 \\
\hline PhytoKeys & 1 \\
\hline Revista Chapingo Serie Forestales y del Ambiente & 1 \\
\hline Revista Mexicana de Biodiversidad & 4 \\
\hline Rhodora & 1 \\
\hline Selbyana & 1 \\
\hline Libros & 20 \\
\hline Capítulos de libros & 10 \\
\hline Planes de desarrollo & 1 \\
\hline Floras y fascículos de Floras & 175 \\
\hline Flora Fanerogámica del Valle de México & 1 \\
\hline Flora del Bajío y de regiones adyacentes & 143 \\
\hline Flora de Guerrero & 31 \\
\hline Total & 351 \\
\hline
\end{tabular}


Cuadro 2: Distribución taxonómica de las angiospermas en el Estado de México.

\begin{tabular}{lccccc}
\hline Clases & Familias & Géneros & Especies & $\begin{array}{c}\text { Endémicas de } \\
\text { México }\end{array}$ & $\begin{array}{c}\text { Endémicas del } \\
\text { Estado de México }\end{array}$ \\
\hline Magnoliides & 9 & 13 & 37 & 11 & 0 \\
Monocotiledóneas & 28 & 248 & 1007 & 358 & 13 \\
Eudicotiledóneas & 132 & 842 & 2880 & 1222 & 16 \\
Total & 169 & 1103 & 3924 & 1591 & 29 \\
\hline
\end{tabular}

tamente enfocada a la entidad mexiquense, es decir, documentos estatales, regionales o incluso nacionales: artículos (30), capítulos de libros (2), informes de proyectos (2), libros (11) y tesis (3), además de la Flora Fanerogámica del Valle de México, Flora del Bajío y de regiones adyacentes (143 fascículos) y Flora de Guerrero (31). Los artículos y las tesis destacan por el número de contribuciones (Fig. 2), los primeros concentran el mayor número de angiospermas exclusivas (220), es decir, especies únicamente citadas en este tipo de bibliografía, y las tesis registran $80 \%$ de los taxa presentes en la entidad (3117 especies) (Cuadro 3).

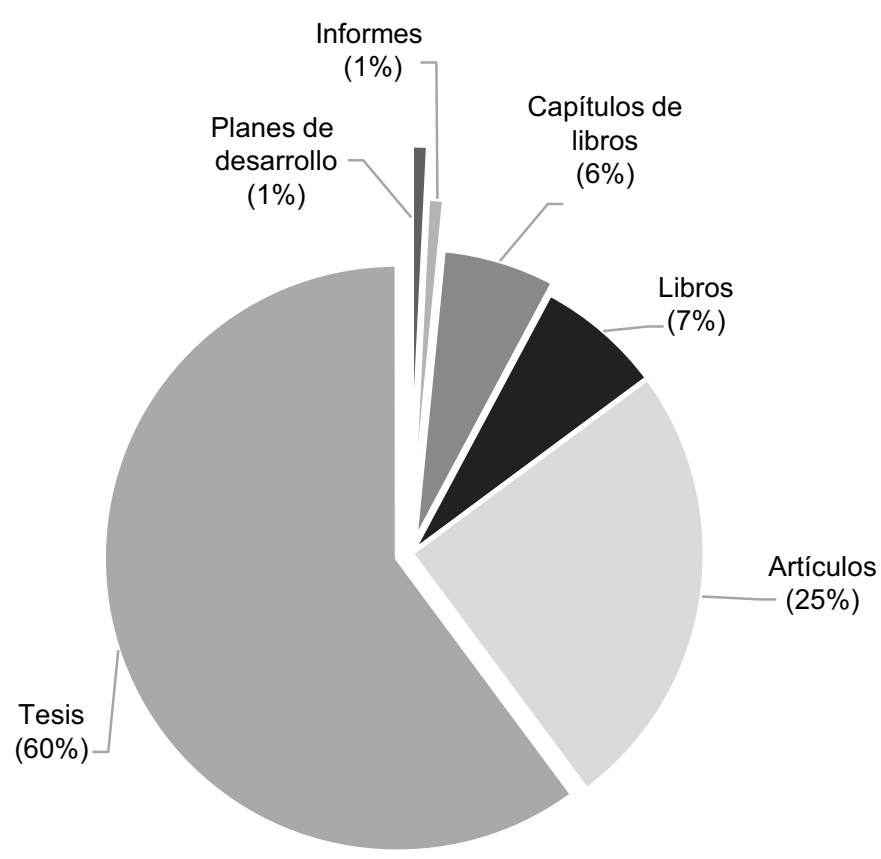

Figura 2: Tipo de publicaciones que documentan las especies de angiospermas del Estado de México.
En el Estado de México, aproximadamente la mitad de las angiospermas (1620 especies) son elementos endémicos de México; entre estas se encuentran 29 endemismos de este estado. Myriophyllum quitense Kunth (Haloragaceae) y Montia fontana $\mathrm{L}$. (Montiaceae) son especies nativas no endémicas que en la República Mexicana se han reportado exclusivamente en la entidad mexiquense. Lachemilla mexiquense D.F. Morales-B. (Rosaceae) no se citó en la publicación de Villaseñor (2016).

La riqueza de especies documentada en la presente contribución incrementó en 55.2\% el número registrado por Martínez y Matuda (1979), en 20.3\% la cifra reportada por Garduño-Solórzano et al. (2009) y en 3.3\% lo reportado por Villaseñor y Ortiz (2014) (Cuadro 4). Sin embargo, existe una diferencia de 928 especies (19.1\%) con respecto a las 4852 plantas con flores documentadas recientemente por Villaseñor (2016).

En los diferentes recuentos para el Estado de México es notoria la riqueza de Asteraceae con respecto a otras familias botánicas. Alrededor de $15 \%$ del total de especies registradas para la entidad mexiquense corresponden a elementos de esta familia; otro $10 \%$ pertenece a Fabaceae, la cual ocupa el segundo lugar en cuanto a número de angiospermas. Aproximadamente $9 \%$ de los taxa son de la familia Poaceae y $6 \%$ Orchidaceae, familias que ocupan la tercera y cuarta posición. En la familia Asteraceae, los géneros más diversos son Ageratina Spach (42 especies) y Stevia Cav. (36); en Fabaceae, Desmodium Desv. (42) y Dalea L. (28); en Poaceae, Muhlenbergia Schreb. (54) y Paspalum L. (30), y en Orchidaceae, Habenaria Willd. y Malaxis Sol. ex Sw. (22 especies cada uno). 
Cuadro 3: Número de angiospermas reportadas para el Estado de México por tipo de publicación. Especies exclusivas se refiere a solamente citadas en ese tipo de publicación.

\begin{tabular}{lcc}
\hline Publicación & Especies & Especies exclusivas \\
\hline Literatura específica para el estado & $\mathbf{3 6 5 7}$ & $\mathbf{5 4 6}$ \\
Literatura no publicada (gris) & $\mathbf{3 1 3 0}$ & $\mathbf{2 1 4}$ \\
Informes de proyectos & 254 & 1 \\
Planes de desarrollo & 376 & 4 \\
Tesis & 3117 & 209 \\
Literatura publicada & $\mathbf{3 1 2 5}$ & $\mathbf{3 3 2}$ \\
Artículos & 2360 & 220 \\
Libros & 1897 & 43 \\
Capítulos de libros & 1196 & 69 \\
Literatura no específica para el estado & $\mathbf{1 8 2 3}$ & $\mathbf{1 4 4}$ \\
Literatura no publicada & $\mathbf{3 4}$ & $\mathbf{2}$ \\
Informes de proyectos & 22 & 0 \\
Tesis & 12 & 2 \\
Literatura publicada & $\mathbf{1 8 0 7}$ & $\mathbf{1 4 2}$ \\
Artículos & 717 & 93 \\
Capítulos de libros & 476 & 28 \\
Libros & 1048 & $\mathbf{6 3}$ \\
Floras & $\mathbf{1 9 1 0}$ & 36 \\
Flora del Bajío y de regiones adyacentes & 992 & 13 \\
Flora de Guerrero & 208 & \\
Flora Fanerogámica del Valle de México & 1258 & \\
\hline & & 21 \\
\hline
\end{tabular}

Cuadro 4: Comparación de la riqueza de angiospermas reportada para el Estado de México. ND = información no disponible.

\begin{tabular}{lccccc}
\hline Referencia bibliográfica & Especies & Asteraceae & Fabaceae & Poaceae & Orchidaceae \\
\hline Martínez y Matuda (1979) & 2528 & 354 & 258 & 271 & 110 \\
Galicia-Miranda (1992) & 2162 & 390 & 215 & 205 & 63 \\
Alcántara-Alameda (2006) & 3937 & 663 & 358 & 411 & 112 \\
Garduño-Solórzano et al. (2009) & 3262 & 495 & 416 & 405 & 181 \\
Villaseñor y Ortiz (2014) & 3799 & ND & ND & ND & ND \\
Villaseñor (2016) & 4852 & 726 & 455 & 395 & 243 \\
Presente estudio & 3924 & 588 & 372 & 340 & 231 \\
\hline
\end{tabular}


En el Estado de México, 93.3\% de los géneros (1029 taxa) tienen de una a 10 especies, 4.6\% (51) concentraron de 11 a $18,1.3 \%$ (14) de 19 a 37, mientras que solamente $0.8 \%$ (9) resultaron más diversos al reunir de 38 a 54 especies. Tales resultados manifiestan alta riqueza de géneros, pero baja diversidad de especies. Los géneros con mayor riqueza específica son Muhlenbergia, Salvia L. (Lamiaceae), Ipomoea L. (Convolvulaceae), Euphorbia L. (Euphorbiaceae) y Ageratina (Asteraceae) (Cuadro 5).

La consulta de literatura florística elaborada en el territorio del Estado de México mostró que se han llevado a cabo al menos 128 trabajos en el transcurso de 38 años, lo que refleja una tendencia de incremento a través del tiempo (Fig. 3). En promedio, se registraron 3.3 inventarios por año.

La distribución de los sitios de exploración botánica en el Estado de México no es uniforme; se han llevado a cabo más trabajos florísticos en la región sur y suroeste, así como en la parte norte de la entidad, particularmente en los límites con Hidalgo y en la Zona Metropolitana del Valle Cuautitlán-Texcoco (porción noreste y oriente). La cuenca alta del río Lerma (región noroeste y el Valle de Toluca) registró menor número de inventarios. Las áreas pobremente recolectadas corresponden a regiones montañosas de acceso problemático (Fig. 4).
Aproximadamente tres cuartas partes de los inventarios incluyeron un área de exploración botánica menor de $95 \mathrm{~km}^{2}$, la cuarta parte restante abarca una superficie mayor. Entre las contribuciones que sobresalen por la riqueza de especies, así como por la superficie de estudio, están los trabajos realizados por López-Patiño et al. (2012) en el Área Natural Protegida "Tenancingo, Malinalco, Zumpahuacán” (LP12), Jiménez-Cruz (2012) en la Sierra de Tepotzotlán (JC12), Castañeda-Robles (2008) en el Cerro Zempoala y zonas aledañas (CR08) y Romero-Rangel y Rojas-Zenteno (1991) en la región de Huehuetoca (RR91) (Fig. 5).

Es notable destacar que los sitios que consideraron menor superficie de exploración florística presentaron mayor diversidad (Fig. 6). Lo anterior manifiesta que en el territorio mexiquense existe una importante cantidad de especies en cada kilómetro cuadrado y particularmente en las zonas protegidas por la orografía. Destaca, por ejemplo, la publicación de López-Sandoval et al. (2010) en la barranca de Nenetzingo (Ixtapan de la Sal), cuya diversidad es de 747 especies por unidad de área, el mayor de todos los evaluados.

En la frecuencia de angiospermas en los inventarios del Estado de México (Fig. 7), aproximadamente un tercio se reportan en una o dos publicaciones; $18.1 \%$ de las

Cuadro 5: Géneros con mayor número de especies en el Estado de México.

\begin{tabular}{llll}
\hline Géneros (Eudicotiledóneas) & Especies & Géneros (Monocotiledóneas) & Especies \\
\hline Salvia L. & 53 & Muhlenbergia Schreb. & 54 \\
Ipomoea L. & 51 & Tillandsia L. & 38 \\
Euphorbia L. & 45 & Cyperus L. & 37 \\
Ageratina Spach & 42 & Carex L. & 30 \\
Desmodium Desv. & 42 & Paspalum L. & 30 \\
Quercus L. & 41 & Dioscorea L. & 26 \\
Solanum L. & 39 & Bouteloua Lag. & 22 \\
Stevia Cav. & 36 & Habenaria Willd. & 22 \\
Bursera Jacq. ex L. & 30 & Malaxis Sol. ex Sw. & 22 \\
Dalea L. & 28 & Bletia Ruiz \& Pav. & 16 \\
\hline
\end{tabular}




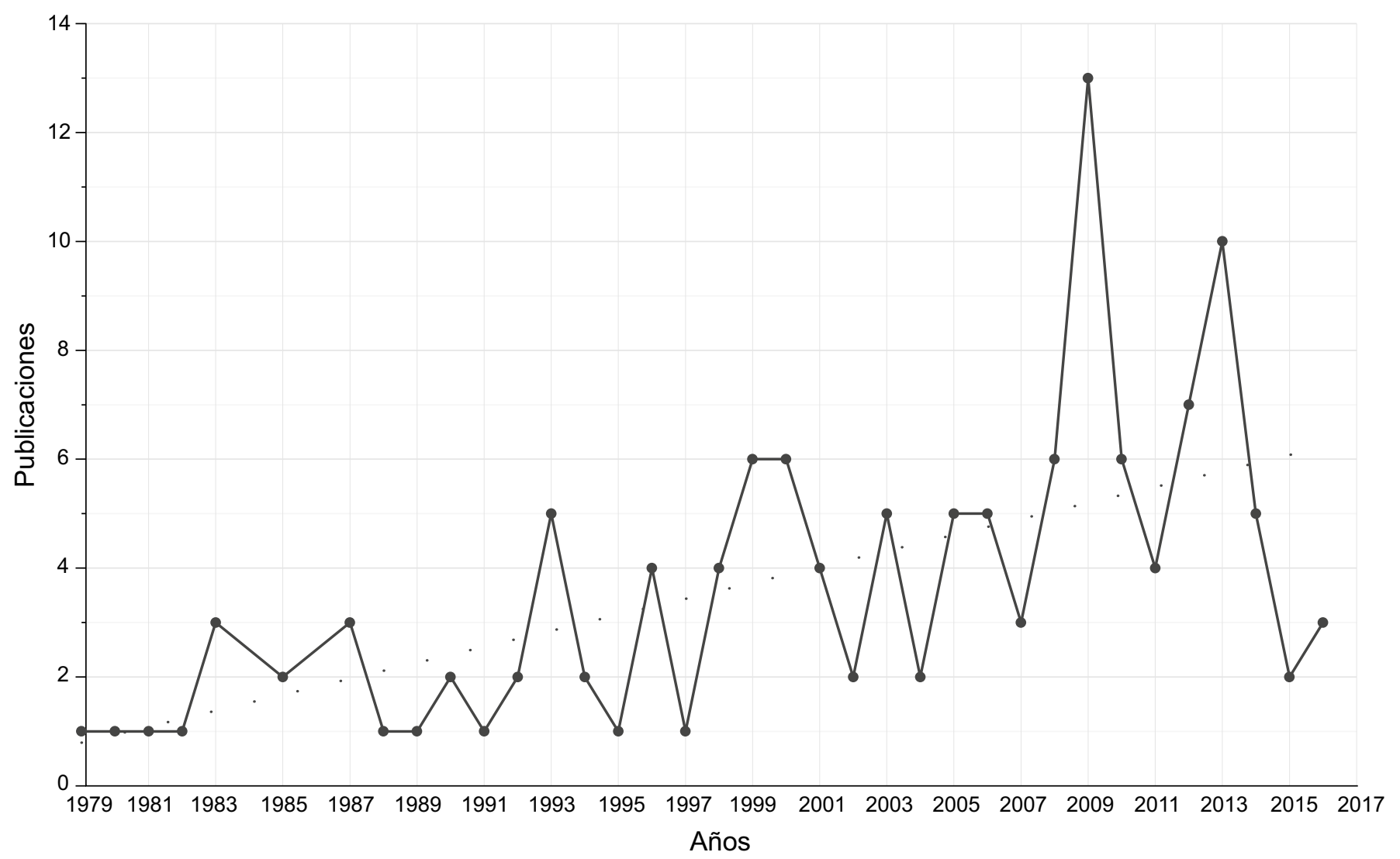

Figura 3: Número de trabajos florísticos llevados a cabo en el Estado de México, distribuidos por año.

especies (714 taxa) se documentan en alguno de los 128 catálogos y 11.6\% (456) se reportan únicamente en dos. Por otra parte, algunas especies están ampliamente distribuidas en el Estado de México debido a que están presentes en más de una tercera parte del total de inventarios: Lopezia racemosa Cav. (60 catálogos), Prunus serotina Ehrh. (57), Barkleyanthus salicifolius (Kunth) H. Rob. \& Brettell (54), Phytolacca icosandra L. (53), Oxalis corniculata L. (52), Dahlia coccinea Cav. (51), Arbutus xalapensis Kunth (49), Geranium seemannii Peyr. (48), Cosmos bipinnatus Cav. (48), Oenothera rosea L'Hér. ex Aiton (48), Tagetes lucida Cav. (48), Baccharis conferta Kunth (47), Begonia gracilis Kunth (47), Bidens odorata Cav. (47), Cuphea aequipetala Cav. (46), Trifolium amabile Kunth (46), Bouvardia ternifolia (Cav.) Schltdl. (45), Crataegus mexicana DC. (45) y Verbena carolina L. (45).

Se registran bajos niveles de similitud florística entre los inventarios (Fig. 8), lo cual revela que la ma- yoría de las especies están reportadas en una o pocas compilaciones. Los catálogos llevados a cabo en áreas montañosas tienden a presentar mayor semejanza (0.59 similitud); por ejemplo, el complejo montañoso Tetzcutzingo (que incluye los Cerros Tetzcutzingo (P92) (Pulido y Koch, 1992) y Metecatl (HC16) (Hernández Cruz et al., 2016)).

\section{DISCUSIÓN}

La diferencia entre la riqueza de angiospermas en familias exitosas en la entidad (Asteraceae, Fabaceae y Poaceae) probablemente se deba a la información indicada en los ejemplares de herbario y nunca citada en la literatura. Un problema más lo constituye el hecho de que se trata de familias con especies difíciles de determinar mediante la literatura disponible; además, en ocasiones los ejemplares carecen de estructuras vegetativas o reproductivas (flores $\mathrm{y}$ frutos), por lo que a veces se reportan solamente a nivel 


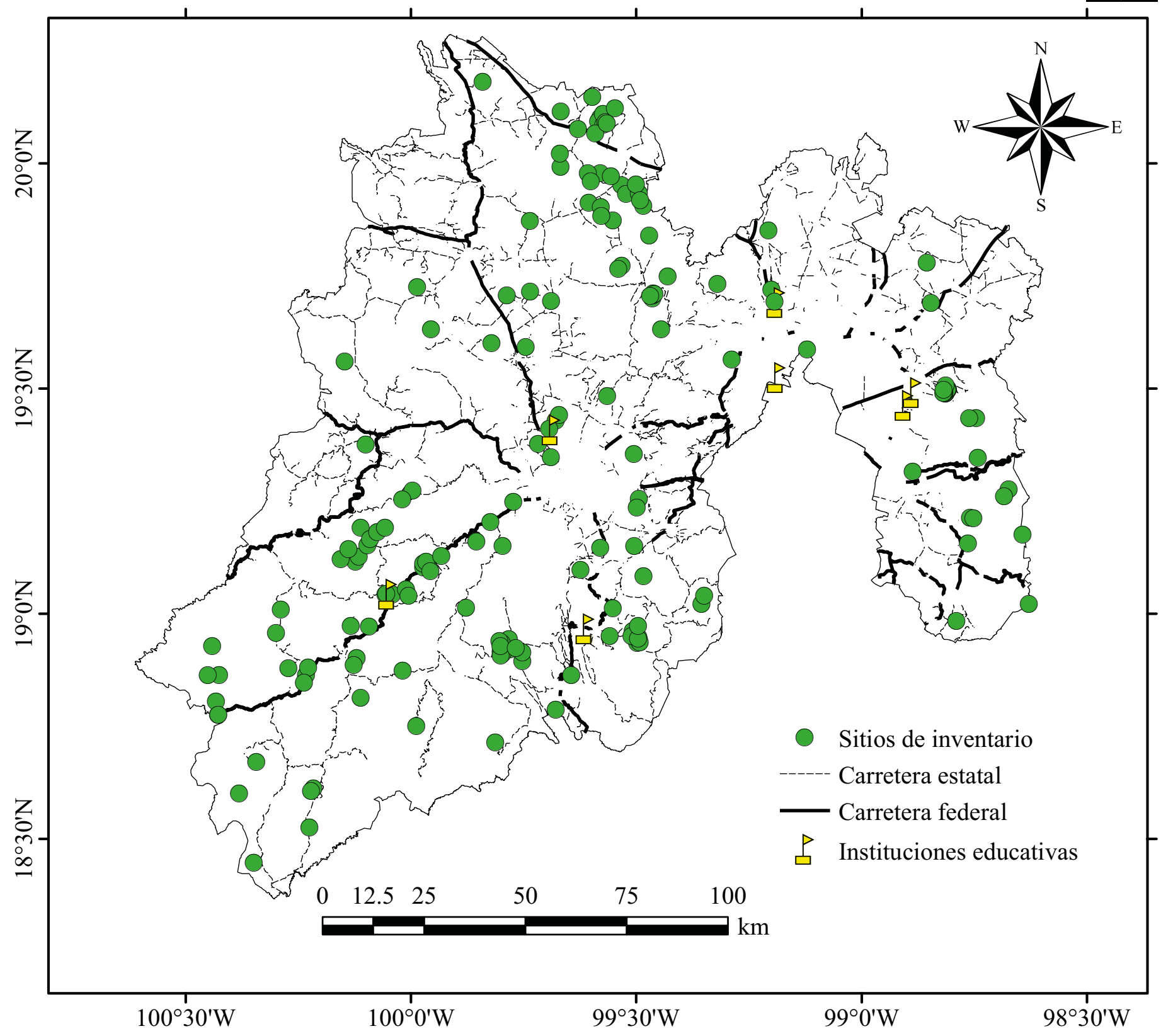

Figura 4: Sitios de exploración botánica en el Estado de México.

de género (Alcántara-Alameda, 2006). Lo anterior marca la necesidad de profundizar en el estudio taxonómico de estas familias.

La información disponible en la literatura no publicada (gris) complementa la que se presenta en la literatura publicada y fácilmente consultable. Una importante cantidad de especies (209) está documentada exclusivamente a partir de tesis de licenciatura y posgrado.
La mayor exploración botánica se ha realizado en zonas adyacentes a vías de comunicación e instituciones importantes. Lo anterior puede explicarse en parte por la facilidad de acceso a estas áreas geográficas (GonzálezTamayo, 2000; Llorente-Bousquets y Ocegueda, 2008).

El índice de diversidad de Squeo et al. (1998) reflejó que las zonas con mayor área de exploración botánica no necesariamente presentan una considerable cantidad de 


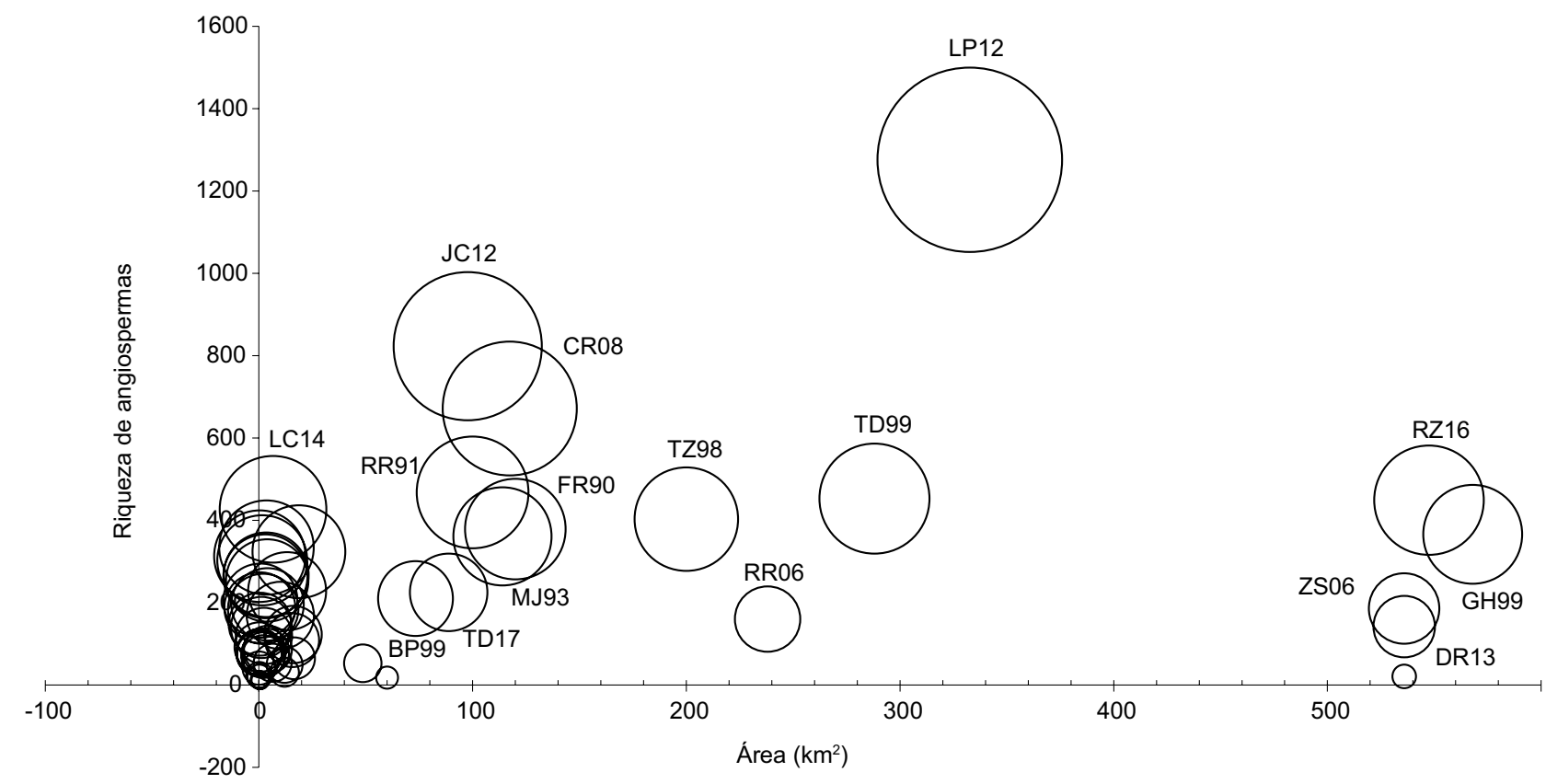

Figura 5: Riqueza de angiospermas por área de estudio en los inventarios florísticos del Estado de México. FR90: Fragoso-Ramírez (1990), RR91: Romero-Rangel y Rojas-Zenteno (1991), MJ93: Miranda-Jiménez y González-Ortiz (1993), TZ98: Torres-Zúñiga y Tejero (1998), BP99: Bernal-Pedreira (1999), GH99: García-Hernández (1999), TD99: Tejero-Díez y Castilla-Hernández (1999), RR06: Romero-Romero (2006), ZS06: Zúñiga-Soto (2006), CR08: Castañeda-Robles (2008), JC12: Jiménez-Cruz (2012), LP12: López-Patiño et al. (2012), DR13: Díaz-Roldán (2013), LC14: Luna-Céspedes (2014), RZ16: Rojas-Zenteno et al. (2016) y TD17: Trejo-Díaz y Tejero-Díez (2017).

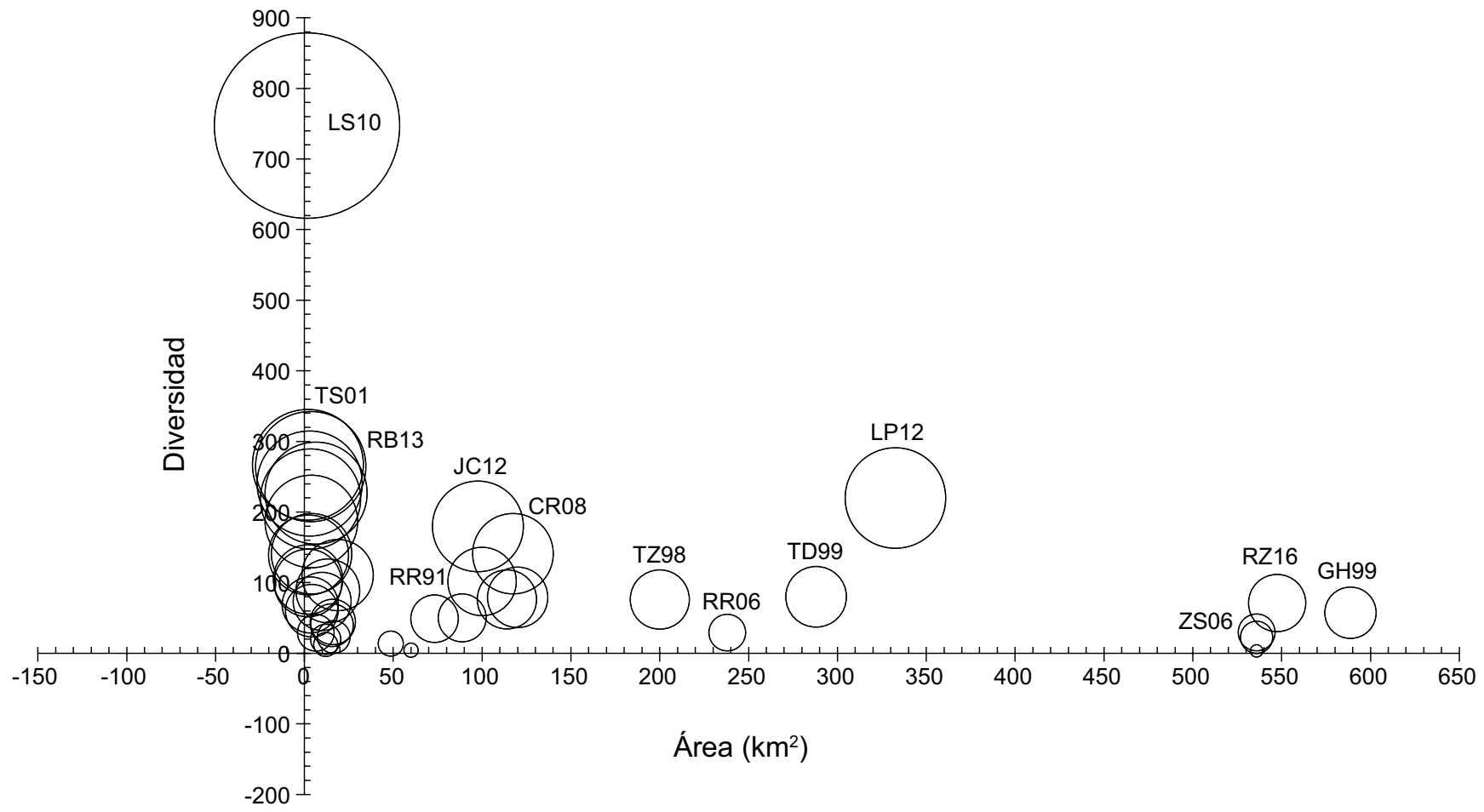

Figura 6: Diversidad de angiospermas en los inventarios florísticos del Estado de México. Índice de diversidad (ID)=especies/logaritmo natural del área en kilómetros cuadrados. RR91: Romero-Rangel y Rojas-Zenteno (1991), TZ98: Torres-Zúñiga y Tejero (1998), GH99: García-Hernández (1999), TD99: Tejero-Díez y Castilla-Hernández (1999), TS01: Torres-Soria (2001), RR06: Romero-Romero (2006), ZS06: Zúñiga-Soto (2006), CR08: Castañeda-Robles (2008), LS10: López-Sandoval et al. (2010), JC12: Jiménez-Cruz (2012), LP12: López-Patiño et al. (2012), RB13: Rodríguez-Barquet y Rodríguez-Sánchez (2013) y RZ16: Rojas-Zenteno et al. (2016). 


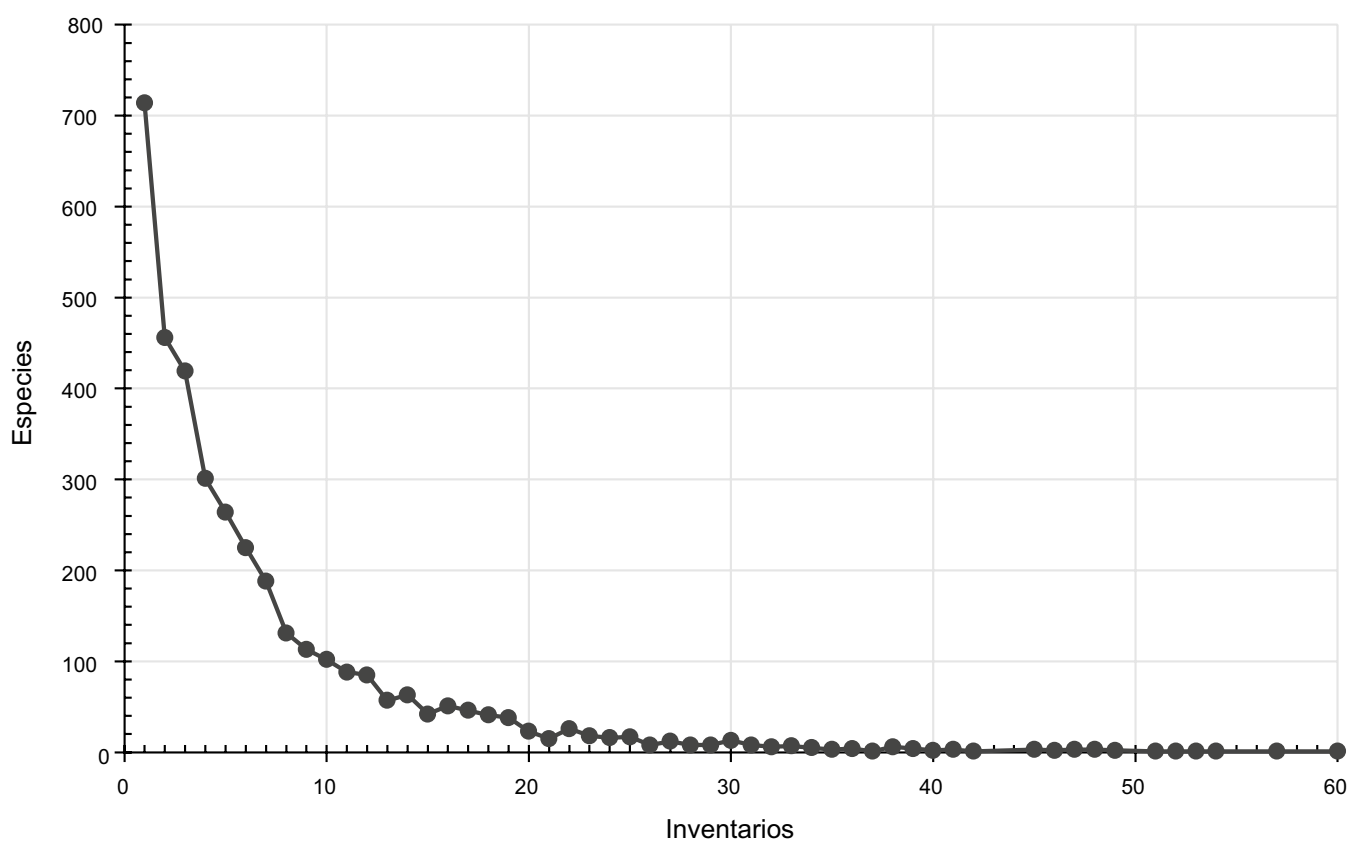

Figura 7: Frecuencia de angiospermas en los inventarios florísticos del Estado de México.

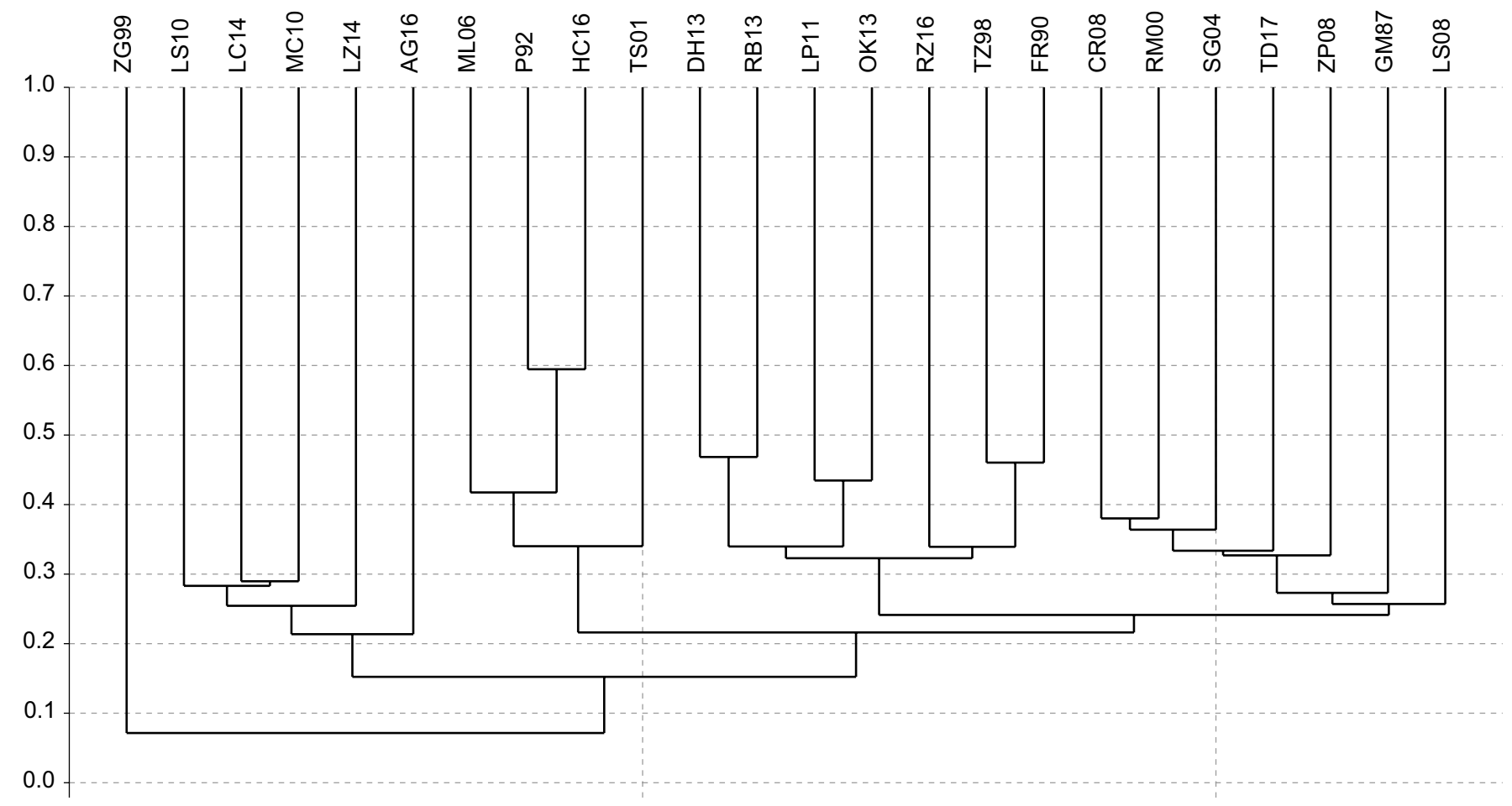

Figura 8: Similitudes florísticas entre los inventarios llevados a cabo en el Estado de México. Coeficiente de similitud utilizado: Sørensen-Dice; método de agrupamiento: UPGMA. GM87: González-Martínez y Rodríguez-Zaragoza (1987), FR90: Fragoso-Ramírez (1990), P92: Pulido y Koch (1992), TZ98: Torres-Zúñiga y Tejero (1998), ZG99: Zepeda-Gómez y Velázquez-Montes (1999), RM00: Rivas-Manzano y Vibrans (2000), TS01: Torres-Soria (2001), SG04: Sánchez-González (2004), ML06: Medina-Lemus y Tejero-Díez (2006), CR08: Castañeda-Robles (2008), LS08: López-Sandoval et al. (2008), ZP08: Zepeda-Peña (2008), LS10: López-Sandoval et al. (2010), MC10: Martínez-De La Cruz (2010), LP11: López-Pérez et al. (2011), DH13: Dorantes-Hernández y Piña-Dorantes (2013), OK13: Ochoa-Kato (2013), RB13: Rodríguez-Barquet y Rodríguez-Sánchez (2013), LC14: Luna-Céspedes (2014), LZ14: López-Zamora (2014), AG16: Aguilera-Gómez et al. (2016), HC16: HernándezCruz et al. (2016), RZ16: Rojas-Zenteno et al. (2016) y TD17: Trejo-Díaz y Tejero-Díez (2017). 
angiospermas por unidad de superficie. Lo anterior puede estar relacionado a que una exploración botánica en zonas pequeñas facilita una revisión a detalle, a diferencia de extensiones grandes, donde generalmente se muestrean sitios puntuales. Aunado a esto, espacios con topografía accidentada favorecen la presencia de microclimas que propician el desarrollo de alto número de plantas con flores (López-Sandoval et al., 2010).

Es necesario continuar el inventario de las plantas con flores, debido a que las exploraciones botánicas no se han realizado de forma uniforme y aún faltan regiones del estado por recolectar. Entre ellas destaca la cuenca alta del río Lerma, que posee una importante riqueza florística, gracias a la variedad de altitudes (3000-4680 m), las cuales originan microclimas que favorecen la diversidad en la zona. Aquí también existen fallas geológicas (Perales, Pastores o Toxi y Acambay), que constituyen zonas de importancia histórica y favorecen el endemismo biológico (SEMARNAT, 2010).

Existen altos niveles de similitud florística entre los inventarios desarrollados en sitios con bosque húmedo (mesófilo) de montaña (FR90: Fragoso-Ramírez, 1990; TZ98: Torres-Zúñiga y Tejero, 1998; LP11: López-Pérez et al., 2011; DH13: Dorantes-Hernández y Piña-Dorantes, 2013; OK13: Ochoa-Kato, 2013; RB13: Rodríguez-Barquet y Rodríguez-Sánchez, 2013; RZ16: Rojas-Zenteno et al., 2016) ubicados a partir de la zona de transición entre el clima templado y cálido, donde prevalecen elevadas condiciones de humedad, que permiten el desarrollo de especies propias de este tipo de vegetación. Los inventarios de la zona sur y suroeste de la entidad se agruparon seguramente por su afinidad ecológica, ya que se llevaron a cabo en sitios con bosque tropical caducifolio (LS10: López-Sandoval et al., 2010; MC10: Martínez-De La Cruz, 2010; LZ14: López-Zamora, 2014; LC14: LunaCéspedes, 2014), y la presencia del bosque templado en la mayor parte del territorio mexiquense influyó en la agrupación de aquellos realizados en el norte del estado y en la cuenca alta del río Lerma (GM87: González-Martínez y Rodríguez-Zaragoza, 1987; LS08: López-Sandoval et al., 2008; ZP08: Zepeda-Peña, 2008; TD17: Trejo-Díaz y Tejero-Díez, 2017) y en la Zona Metropolitana del Va1le Cuautitlán-Texcoco (P92: Pulido y Koch, 1992; TS01: Torres-Soria, 2001; ML06: Medina-Lemus y Tejero-Díez, 2006; HC16: Hernández-Cruz et al., 2016).

La información sobre la riqueza de angiospermas, acumulada hasta la fecha en la literatura consultada, resulta fundamental para determinar posteriormente la distribución geográfica de las especies en los municipios del Estado de México. Además, permite adicionar información sobre endemismo, usos (actuales y potenciales) de las plantas y establecer programas de conservación de los recursos naturales. El Estado de México es una entidad con importancia florística por la alta riqueza de angiospermas, la presencia de endemismos, así como por el número de especies no endémicas pero exclusivas del territorio mexiquense. Como sugieren Suárez-Mota y Villaseñor (2011) para Oaxaca, el Estado de México es también una región irremplazable y las estrategias orientadas a la conservación de su diversidad biológica necesitan considerar en primera instancia a las zonas con endemismos y los sitios con especies raras, pues son grupos prioritarios para la conservación, debido a su vulnerabilidad a la extinción. Muchas de esas zonas tienen alguna referencia bibliográfica (publicada o no), que destaca su valor científico, cultural y biológico.

\section{CONTRIBUCIÓN DE AUTORES}

IMC y JLV concibieron, diseñaron el estudio e integraron la base de datos de las angiospermas. IMC, LAG y MRA contribuyeron en la recopilación de literatura florísticataxonómica para integrar la base de datos. JLV orientó el análisis. IMC realizó el análisis de datos. IMC redactó el manuscrito en colaboración con MRA y LAG. Todos los autores contribuyeron en la revisión y aprobación del manuscrito final.

\section{FINANCIAMIENTO}

Este estudio fue apoyado por el Consejo Nacional de Ciencia y Tecnología (CONACYT) con la beca (211499) otorgada a la primera autora para realizar estudios de posgrado, y por la Universidad Autónoma del Estado de México, mediante el proyecto de investigación 3765/2014/CID. 


\section{AGRADECIMIENTOS}

A Maricela Rodríguez Acosta y Allen James Coombes por las observaciones emitidas en la integración de la lista de angiospermas. A Ricardo Vega Muñoz por facilitar la consulta de literatura florística-taxonómica en el Herbario-Hortorio (CHAPA) del Colegio de Postgraduados. A Mario Nava Reyes del Instituto Nacional de Estadística y Geografía por la asesoría en el programa Mapa Digital de México. A Irma Victoria Rivas Manzano y Miguel García Ortiz por el apoyo constante. A los revisores anónimos por los comentarios y sugerencias al manuscrito.

\section{LITERATURA CITADA}

Aguilera-Gómez, L. I., I. V. Rivas-Manzano, I. Martínez-De La Cruz y C. J. Aguilar-Ortigoza. 2016. El bosque tropical subcaducifolio en dos cañadas de Tlatlaya, Estado de México. Polibotánica 41: 1-29. DOI: https://dx.doi. org/10.18387/polibotanica.41.1

Alcántara-Alameda, D. 2006. Base de datos de pteridofitas, gimnospermas y angiospermas del Estado de México. Tesis de licenciatura. Facultad de Ciencias Agrícolas, Universidad Autónoma del Estado de México. Toluca, Estado de México, México. 165 pp.

APG IV. 2016. An update of the Angiosperm Phylogeny Group classification for the orders and families of flowering plants: APG IV. Botanical Journal of the Linnean Society 181(1): 1-20. DOI: https://doi.org/10.1111/boj.12385

Badii, M. H., J. Landeros y E. Cerna. 2008. Patrones de asociación de especies y sustentabilidad. Daena: International Journal of Good Conscience 3(1): 632-660.

Bernal-Pedreira, M. de los A. A. 1999. Guía ilustrada de la Sierra de Guadalupe. Tesis de licenciatura. Facultad de Estudios Superiores Iztacala, Universidad Nacional Autónoma de México. Tlalnepantla, Estado de México, México. 254 pp.

Calderón de Rzedowski, G. y J. Rzedowski (eds.). 2005. Flora Fanerogámica del Valle de México. Instituto de Ecología, A.C. y Comisión Nacional para el Conocimiento y Uso de la Biodiversidad. Pátzcuaro, México. 1406 pp.

Castañeda-Robles, J. 2008. Estudio florístico del Cerro de Zempoala y zonas aledañas, municipio de Ocuilan, Edo. de México. Tesis de licenciatura. Facultad de Estudios Superiores Iztacala, Universidad Nacional Autónoma de México. Tlalnepantla, Estado de México, México. 37 pp.

Díaz-Roldán, A. V. 2013. Diagnósis de la vegetación y flora del Parque Nacional Nevado de Toluca (Estado de México, México) y propuesta para su gestoría. Tesis de licenciatura. Facultad de Estudios Superiores Iztacala, Universidad Nacional Autónoma de México. Tlalnepantla, Estado de México, México. 71 pp.

Diego-Pérez, N. y R. M. Fonseca (eds.). 1995-2014. Flora de Guerrero. Facultad de Ciencias, Universidad Nacional Autónoma de México. México, D.F., México. 1208 pp.

Dorantes-Hernández, F. e I. V. Piña-Dorantes. 2013. Estudio del ecotono del bosque de encino y tropical caducifolio en la cuenca del río Tlapala, Coatepec Harinas, Estado de México, México. Tesis de licenciatura. Facultad de Estudios Superiores Iztacala, Universidad Nacional Autónoma de México. Tlalnepantla, Estado de México, México. 107 pp.

Espejo-Serna, A. 2012. El endemismo en las Liliopsida mexicanas. Acta Botanica Mexicana 100: 195-257. DOI: https://dx.doi.org/10.21829/abm100.2012.36

Espejo-Serna, A. y A. R. López-Ferrari. 2004. Checklist of Mexican Bromeliaceae with notes on species distribution and levels of endemism. Selbyana 25(1): 33-86.

Espinosa-García, F. J. 2000. Malezas introducidas en México, proyecto No. U024. Centro de Investigaciones en Ecosistemas, Universidad Nacional Autónoma de México. Informe final Sistema Nacional de Información sobre Biodiversidad (SNIB)-Comisión Nacional para el Conocimiento y Uso de la Biodiversidad (CONABIO). México, D.F., México.

ESRI. 2013. ArcGIS Desktop 10.2. Environmental Systems Research Institute. Redlands, USA.

Fragoso-Ramírez, R. 1990. Estudio florístico en la parte alta de la Sierra de Zacualpan, Estado de México. Tesis de licenciatura. Facultad de Estudios Superiores Iztacala, Universidad Nacional Autónoma de México. Tlalnepantla, Estado de México, México. 69 pp.

Galicia-Miranda, M. V. 1992. Listado florístico del Estado de México y regiones circundantes (de los estados de Hidalgo, 
Querétaro y Distrito Federal) basado en las colecciones de Eizi Matuda. Tesis de licenciatura. Facultad de Ciencias, Universidad Nacional Autónoma de México. México, D.F., México. 207 pp.

García-Hernández, A. R. 1999. Inventario florístico del municipio de Jilotepec, Estado de México. Tesis de licenciatura. Facultad de Estudios Superiores Iztacala, Universidad Nacional Autónoma de México. Tlalnepantla, Estado de México, México. 105 pp.

García-Mendoza, A. J. y J. A. Meave (eds.). 2012. Diversidad florística de Oaxaca: de musgos a angiospermas (colecciones y lista de especies). Universidad Nacional Autónoma de México (UNAM)-Comisión Nacional para el Conocimiento y Uso de la Biodiversidad (CONABIO)Instituto Estatal de Ecología y Desarrollo Sustentable. Cd. Mx., México. 351 pp.

Garduño-Solórzano, G., R. López-Cano, D. A. MonterrubioPasapera y A. de La Rosa-Segura. 2009. Sinopsis de la flora. In: Ceballos, G., R. List, G. Garduño, R. LópezCano, M. J. Muñozcano, E. Collado y J. E. San Román (comps.). La diversidad biológica del Estado de México. Estudio de Estado. Gobierno del Estado de México. Toluca, México. Pp. 261-274.

González-Martínez, F. y S. Rodríguez-Zaragoza. 1987. Estado actual de la vegetación de un área de bosque de encinopino en Villa del Carbón, Estado de México. Tesis de licenciatura. Facultad de Estudios Superiores Iztacala, Universidad Nacional Autónoma de México. Tlalnepantla, Estado de México, México. $91 \mathrm{pp}$.

González-Tamayo, R. 2000. Una especie nueva de Malaxis (Orchidaceae) del Estado de México. Boletín del Instituto de Botánica de la Universidad de Guadalajara 8(1-2): 175-179.

Google Inc. 2009. Google Earth. Version 6.2. (Software). http:// www.google.com/earth/ (consultado junio de 2017).

Hammer, Ø., D. A. T. Harper y P. D. Ryan. 2001. PAST: paleontological statistics software package for education and data analysis. Paleontologia Electronica 4(1): 1-9.

Hernández-Cruz, M. J., S. D. Koch-Olt, M. T. P. Pulido-Salas, M. Luna-Cavazos y E. García-Villanueva. 2016. Estudio florístico del cerro Metecatl, del complejo montañoso Tetzcutzingo, Texcoco, Estado de México, México.
Botanical Sciences 94(2): 377-392. DOI: https://dx.doi. org/10.17129/botsci.240

Ibarra-Manríquez, G., J. L. Villaseñor y R. Durán-García. 1995. Riqueza de especies y endemismo del componente arbóreo de la Península de Yucatán, México. Boletín de la Sociedad Botánica de México 57: 49-77.

INEGI. 2001. Síntesis de Información Geográfica del Estado de México, nomenclátor y anexo cartográfico. Instituto Nacional de Estadística, Geografía e Informática. Aguascalientes, México.

INEGI. 2011. Perspectiva estadística: México. Instituto Nacional de Estadística y Geografía. México, D.F., México.

INEGI. 2013. Sistema para la Consulta de Información Censal (SCINCE Versión 05/2012). http://www.inegi.org.mx/est/ scince/scince2010.aspx (consultado mayo de 2017).

INEGI. 2016a. Anuario estadístico y geográfico de México. Instituto Nacional de Estadística y Geografía. Aguascalientes, México.

INEGI. 2016b. Anuario estadístico y geográfico de los Estados Unidos Mexicanos. Instituto Nacional de Estadística y Geografía. Aguascalientes, México.

INEGI. 2017. Mapa Digital de México. Versión 6.3.0. Instituto Nacional de Estadística y Geografía. Aguascalientes, México. http://www.inegi.org.mx/geo/contenidos/ mapadigital/ (consultado mayo de 2017).

IPNI. 2016. The International Plant Name Index. Publicado en internet http://www.ipni.org (consultado mayo de 2016).

Jiménez-Cruz, A. 2012. Estudio florístico del parque estatal Sierra de Tepotzotlán, Estado de México. Tesis de licenciatura. Facultad de Estudios Superiores Iztacala, Universidad Nacional Autónoma de México. Tlalnepantla, Estado de México, México. 103 pp.

Llorente-Bousquets, J. y S. Ocegueda. 2008. Estado del conocimiento de la biota. In: Ocegueda, S. y J. LlorenteBousquets (coords.). Capital natural de México, vol. I: Conocimiento actual de la biodiversidad. Comisión Nacional para el Conocimiento y Uso de la Biodiversidad (CONABIO). México, D.F., México. Pp. 283-322.

López-Cano, R., G. Becerril-Zepeda, C. Benítez y S. CuevasSolórzano. 2009. El medio físico, biológico y social. In: 
Ceballos, G., R. List, G. Garduño, R. López-Cano, M. J. Muñozcano, E. Collado y J. E. San Román (comps.). La diversidad biológica del Estado de México. Estudio de Estado. Gobierno del Estado de México. Toluca, México. Pp. 49-61. López-Patiño, E. J., D. R. Szeszko, J. Rescala-Pérez y A. S. Beltrán-Retis. 2012. The flora of the TenancingoMalinalco-Zumpahuacán protected natural area, State of Mexico, Mexico. Harvard Papers in Botany 17(1): 65167. DOI: https://doi.org/10.3100/025.017.0113

López-Pérez, Y., J. D. Tejero-Díez, A. N. Torres-Díaz e I. LunaVega. 2011. Flora del bosque mesófilo de montaña y vegetación adyacente en Avándaro, Valle de Bravo, Estado de México, México. Boletín de la Sociedad Botánica de México 88: 35-53. DOI: https://dx.doi.org/10.17129/ botsci.304

López-Sandoval, J. A., J. I. Valdez-Mercado, L. M. Vázquez-

García, E. J. Morales-Rosales y A. Domínguez-López. 2008. Listado florístico preliminar del Cerro de Jocotitlán, Estado de México. Ciencias Agrícolas Informa 17: 28-42. López-Sandoval, J. A., S. D. Koch, L. M. Vázquez-García, G. Munguía-Lino y E. J. Morales-Rosales. 2010. Estudio florístico de la parte central de la barranca Nenetzingo, municipio de Ixtapan de la Sal, Estado de México. Polibotánica 30: 9-33.

López-Zamora, O. 2014. Composición florística y estructura de la vegetación en dos cañadas de recarga hídrica en Malinalco, Estado de México. Tesis de licenciatura. Facultad de Ciencias, Universidad Autónoma del Estado de México. Toluca, Estado de México, México. 121 pp.

Luna-Céspedes, R. 2014. Flora de las barrancas de Tonatico,

Estado de México, México. Tesis de licenciatura. Facultad de Estudios Superiores Iztacala, Universidad Nacional Autónoma de México. Tlalnepantla, Estado de México, México. 111 pp.

Martínez, M. y E. Matuda. 1979. Flora del Estado de México. Tomos I, II y III. Edición fascimilar de los fascículos publicados en los años de 1953 a 1972. Biblioteca Enciclopédica del Estado de México. México, D.F., México. 1547 pp.

Martínez-De La Cruz, I. 2010. La flora y vegetación ruderal de Malinalco, Estado de México. Tesis de maestría. Colegio de Postgraduados, Campus Montecillo. Texcoco, Estado de México, México. 149 pp.

Medina-Lemus, J. G. y J. D. Tejero-Díez. 2006. Flora y vegetación del parque estatal Atizapán-Valle Escondido. Estado de México, México. Polibotánica 21: 1-43.

Miranda-Jiménez, M. E. y M. A. González-Ortiz. 1993. Estudio de la vegetación y florística de la mesa basáltica de Holotepec, distrito de Tenango del Valle, Estado de México. Tesis de licenciatura. Facultad de Estudios Superiores Iztacala, Universidad Nacional Autónoma de México. Tlalnepantla, Estado de México, México. $74 \mathrm{pp}$.

Neyra-Jáuregui, J. 2012. Altas montañas mexiquenses. Historia natural, turismo y conservación. Consejo Editorial de la Administración Pública Estatal (CEAPE). Toluca, México. 247 pp.

Ochoa-Kato, K. Y. 2013. Flora y estructura de los bosques en "Rancho Cerro Gordo", municipio de Valle de Bravo, Estado de México. Tesis de licenciatura. Facultad de Estudios Superiores Iztacala, Universidad Nacional Autónoma de México. Tlalnepantla, Estado de México, México. $88 \mathrm{pp}$.

POEM. 2009. Decreto número 14 por el que se aprueba la declaratoria de Zona Metropolitana del Valle de Cuautitlán-Texcoco. Periódico Oficial "Gaceta del Gobierno" del Estado de México. Toluca de Lerdo, México. 19 de noviembre de 2009. Tomo CLXXXVIII, número 99. Pp. 14-19. http://legislacion.edomex.gob.mx/ sites/legislacion.edomex.gob.mx/files/files/pdf/gct/2009/ nov193.PDF

POEM. 2016. Decreto número 159 por el que se aprueba la declaratoria de Zona Metropolitana de Santiago Tianguistenco. Periódico Oficial "Gaceta del Gobierno" del Estado de México. Toluca de Lerdo, México. 25 de noviembre de 2016. Tomo CCII, número 103.8 pp. http:// legislacion.edomex.gob.mx/sites/legislacion.edomex. gob.mx/files/files/pdf/gct/2016/nov255.pdf

Pulido, M. T. P. y S. D. Koch. 1992. Guía ilustrada de las plantas del Cerro Tetzcutzingo, especies comunes en el Valle de México. Universidad Nacional Autónoma de México Cuadernos del Instituto de Biología 17: 1-245. 
Pulido-Esparza, V. A., A. Espejo-Serna y A. R. LópezFerrari. 2009. Las monocotiledóneas nativas del corredor biológico Chichinautzin. Acta Botanica Mexicana 86: 9-38. DOI: https://dx.doi.org/10.21829/ abm86.2009.1076

Rivas-Manzano, I. V. y H. Vibrans. 2000. Composición florística de la Unidad de San Cayetano, Villa de Allende, asociada a sitios con distinto tipo de perturbación. Informe Académico de Investigación. Clave: 1383/99. Facultad de Ciencias, Universidad Autónoma del Estado de México. Toluca, Estado de México, México.

Rodríguez-Barquet, L. E. y P. V. Rodríguez-Sánchez. 2013. Estudio del bosque templado húmedo en la cuenca del río Las Flores, Coatepec Harinas, Estado de México. Tesis de licenciatura. Facultad de Estudios Superiores Iztacala, Universidad Nacional Autónoma de México. Tlalnepantla, Estado de México, México. 98 pp.

Rojas-Zenteno, E. C., M. Orozco-Villa, S. Romero-Rangel y R. Montoya-Ayala. 2016. Vegetación y flora del municipio de Temascaltepec, Estado de México, México. Polibotánica 42: 43-89. DOI: https://dx.doi.org/10.18387/ polibotanica. 42.3

Romero-Rangel, S. y E. C. Rojas-Zenteno. 1991. Estudio florístico de la región de Huehuetoca, Estado de México. Acta Botanica Mexicana 14: 33-57. DOI: https://dx.doi. org/10.21829/abm14.1991.613

Romero-Romero, F. 2006. Diversidad biológica, remanentes de vegetación natural y conservación de la cañada de Encinillas en Polotitlán, Estado de México. Tesis de maestría. Facultad de Ciencias, Universidad Nacional Autónoma de México. México, D.F., México. 128 pp.

Roskov, Y., L. Abucay, T. Orrell, D. Nicolson, N. Bailly, P. M. Kirk, T. Bourgoin, R. E. DeWalt, W. Decock, A. De Wever (eds.). 2016. Species 2000 \& ITIS Catalogue of Life, 2016 Annual Checklist. Naturalis Biodiversity Center. Leiden, Netherlands. http://www.catalogueoflife. org/annual-checklist/2016 (consultado mayo de 2016).

Ruiz-Jiménez, C. A., O. Téllez-Valdés e I. Luna-Vega. 2012. Clasificación de los bosques mesófilos de montaña de México: afinidades de la flora. Revista Mexicana de Biodiversidad 83(4): 1110-1144.
Rzedowski, J. y G. Calderón de Rzedowski (eds.). 1991-2014. Flora del Bajío y de regiones adyacentes. Instituto de Ecología, A.C., Centro Regional del Bajío. Pátzcuaro, México.

Sánchez-González, A. 2004. Análisis sinecológico, florístico y biogeográfico de la vegetación del norte de la Sierra Nevada, México. Tesis de doctorado. Colegio de Postgraduados, Campus Montecillo. Texcoco, Estado de México, México. 153 pp.

SEMARNAT. 2010. Inventarios florísticos y faunísticos de la cuenca alta del río Lerma. Plan maestro para la restauración ambiental de la cuenca alta del río Lerma. Secretaría del Medio Ambiente, Gobierno del Estado de México, Universidad Autónoma Chapingo. Toluca, México. 214 pp.

Sotelo-Ruíz, E. D., A. González-Hernández, G. Cruz-Bello, F. Moreno-Sánchez y G. Cruz-Cárdenas. 2011. Los suelos del Estado de México y su actualización a la base referencial mundial del recurso suelo 2006. Revista Mexicana de Ciencias Forestales 2(8): 71-84.

Squeo, F. A., L. A. Cavieres, G. Arancio, J. E. Novoa, O. Matthei, C. Marticorena, R. Rodríguez, M. T. K. Arroyo y M. Muñoz. 1998. Biodiversidad de la flora vascular en la Región de Antofagasta, Chile. Revista Chilena de Historia Natural 71: 571-591.

Suárez-Mota, M. E. y J. L. Villaseñor. 2011. Las compuestas endémicas de Oaxaca, México: diversidad y distribución. Boletín de la Sociedad Botánica de México 88: 55-66. DOI: https://dx.doi.org/10.17129/botsci.308

Tejero-Díez, J. D. y M. E. Castilla-Hernández. 1999. Un método para el estudio del entorno ecológico en las primeras civilizaciones en Mesoamérica. Teotihuacán, un ejemplo. In: González-Jácome, A. y S. del Amo-Rodríguez (comps.). Agricultura y sociedad en México; diversidad, enfoques, estudios de caso. Plaza Valdés, Gestión de Ecosistemas A.C. y Universidad Iberoamericana. México, D.F., México. Pp. 33-60.

Tejero-Díez, J. D. y Ma. de la L. Arreguín-Sánchez. 2004. Lista con anotaciones de los pteridófitos del Estado de México, México. Acta Botanica Mexicana 69: 1-82. DOI: https:// dx.doi.org/10.21829/abm69.2004.981 
The Plant List. 2013. Version 1.1. http://www.theplantlist.org (consultado mayo de 2016).

Torres-Soria, P. 2001. Flora fanerogámica de la zona arqueológica de Teotihuacán, Estado de México. Polibotánica 12: 57-83.

Torres-Zúñiga, Ma. M. y J. D. Tejero. 1998. Flora y vegetación de la Sierra de Sultepec, Estado de México. Anales del Instituto de Biología, Universidad Nacional Autónoma de México, Serie Botánica 69(2): 135-174.

Trejo-Díaz, C. C. y J. D. Tejero-Díez. 2017. Flora de plantas vasculares en la Sierra de las Ánimas, Chapa de Mota, Estado de México, México. Polibotánica 43: 1-35. DOI: https://dx.doi.org/10.18387/polibotanica.43.1

TROPICOS. 2016. Tropicos.org. Missouri Botanical Garden. http://www.tropicos.org (consultado mayo de 2016).

Villaseñor, J. L. 2003. Diversidad y distribución de las Magnoliophyta de México. Interciencia 28(3): 160-167.

Villaseñor, J. L. 2010. El bosque húmedo de montaña en México y sus plantas vasculares: catálogo florístico taxonómico. Comisión Nacional para el Conocimiento y Uso de la Biodiversidad (CONABIO)-Universidad Nacional Autónoma de México (UNAM). Cd. Mx., México. 38 pp.

Villaseñor, J. L. 2016. Checklist of the native vascular plants of México. Revista Mexicana de Biodiversidad 87(3): 559902. DOI: https://dx.doi.org/10.1016/j.rmb.2016.06.017

Villaseñor, J. L. y E. Ortiz. 2014. Biodiversidad de las plantas con flores (División Magnoliophyta) en México. Revista Mexicana de Biodiversidad 85(Suppl.): 134-142. DOI: https://dx.doi.org/10.7550/rmb.31987

Villaseñor, J. L. y F. J. Espinosa-García. 2004. The alien flowering plants of Mexico. Diversity and Distributions
10(2): 113-123. DOI: https://doi.org/10.1111/j.13669516.2004.00059.x

Villaseñor, J. L., E. Ortiz, O. Hinojosa-Espinosa y G. SeguraHernández. 2013. Especies de la familia Asteraceae exóticas a la flora de México. Secretaría de Agricultura, Ganadería, Desarrollo Rural, Pesca y Alimentación (SAGARPA)/Servicio Nacional de Sanidad, Inocuidad y Calidad Agroalimentaria (SENASICA)/Consejo Nacional Consultivo Fitosanitario (CONACOFI)/Universidad Nacional Autónoma de México (UNAM)/Sociedad Mexicana de Ciencia de la Maleza (SOMECIMA). México, D.F, México. 159 pp.

WCSP. 2012. World Checklist of Selected Plant Families. Royal Botanic Gardens, Kew. Kew, UK. http://apps.kew. org/wcsp/home.do (consultado mayo de 2016).

Zepeda-Gómez, C. y E. Velázquez-Montes. 1999. El bosque tropical caducifolio de la vertiente sur de la Sierra de Nanchititla, Estado de México: la composición y la afinidad geográfica de su flora. Acta Botanica Mexicana 46: 29-55. DOI: https://dx.doi.org/10.21829/abm46.1999.815

Zepeda-Peña, N. A. 2008. Flora de San Pedro Arriba, Temoaya, Estado de México. Tesis de licenciatura. Facultad de Ciencias Agrícolas, Universidad Autónoma del Estado de México. Toluca, Estado de México, México. 250 pp.

Zúñiga-Soto, S. N. 2006. Diagnóstico del estudio de la vegetación en el Parque Nacional Nevado de Toluca, Estado de México. Tesis de licenciatura. Facultad de Ciencias, Universidad Nacional Autónoma de México. México, D.F., México. 65 pp. 
Apéndice 1: Literatura gris incluida en la base de datos de las angiospermas del Estado de México.

Adame-Cisneros, J. 1993. Sucesión de arvenses en alfalfares de distintas edades en un rancho lechero en Ixtapaluca, Estado de México. Tesis de licenciatura. Facultad de Estudios Superiores Zaragoza, Universidad Nacional Autónoma de México. México, D.F., México. 111 pp.

Albarrán-Mondragón, F. J. 2009. Estudio florístico de los huertos familiares de la parte sur de Malinalco, Estado de México. Tesis de licenciatura. Facultad de Ciencias, Universidad Autónoma del Estado de México. Toluca, Estado de México, México. 84 pp.

Alcántara-Alameda, D. 2006. Base de datos de pteridofitas, gimnospermas y angiospermas del Estado de México. Tesis de licenciatura. Facultad de Ciencias Agrícolas, Universidad Autónoma del Estado de México. Toluca, Estado de México, México. 165 pp.

Alcántara-Galindo, A. E. 2009. Flora útil de Temoaya, Estado de México. Tesis de licenciatura. Facultad de Estudios Superiores Iztacala, Universidad Nacional Autónoma de México. Tlalnepantla, Estado de México, México. 119 pp.

Alvarado-Moreno, C. 2002. Taxonomía del género Cosmos (Asteraceae, Heliantheae) en el Estado de México. Tesis de licenciatura. Facultad de Ciencias, Universidad Autónoma del Estado de México. Toluca, Estado de México, México. 175 pp.

Bautista-Álvarez, I. 2009. Evaluación de la diversidad de semillas de los sedimentos de la zona inundada de San Pedro Cholula, Estado de México. Tesis de licenciatura. Facultad de Ciencias, Universidad Autónoma del Estado de México. Toluca, Estado de México, México. 79 pp.

Bernal-Pedreira, M. de los A. A. 1999. Guía ilustrada de la Sierra de Guadalupe. Tesis de licenciatura. Facultad de Estudios Superiores Iztacala, Universidad Nacional Autónoma de México. Tlalnepantla, Estado de México, México. 254 pp.

Bolaños-Medina, A. 1996. Los agostaderos forestales del occidente del Estado de México. Tesis de maestría. Colegio de Postgraduados, Campus Montecillo. Texcoco, Estado de México, México. 181 pp.

Carbajal-Esquivel, H. y J. Mondragón-Pichardo. 2000. Diversidad y etnobotánica de la vegetación arvense en la comunidad Mazahua San Pablo Tlalchichilpa, municipio de San Felipe del Progreso, Estado de México. Tesis de licenciatura. Facultad de Ciencias,
Universidad Autónoma del Estado de México. Toluca, Estado de México, México. 104 pp.

Castañeda-Robles, J. 2008. Estudio florístico del cerro de Zempoala y zonas aledañas, municipio de Ocuilan, Edo. de México. Tesis de licenciatura. Facultad de Estudios Superiores Iztacala, Universidad Nacional Autónoma de México. Tlalnepantla, Estado de México, México. 37 pp.

Castillejos-Cruz, C. 2009. Sistemática del género Manfreda Salisb. (Agavaceae). Tesis de doctorado. Colegio de Postgraduados, Campus Montecillo. Texcoco, Estado de México, México. 331 pp.

Castro-Luna, M. 2013. Registro de la riqueza herbácea y arbustiva en el bosque de Abies religiosa de la zona de amortiguamiento del parque nacional Izta-Popo y el parque nacional Zoquiapan. Tesis de licenciatura. Facultad de Estudios Superiores Zaragoza, Universidad Nacional Autónoma de México. México, D.F., México. 179 pp.

Cazares-Mena, A. 1994. Catálogo de plantas medicinales del Estado de México. Tesis de licenciatura. Facultad de Estudios Superiores Iztacala, Universidad Nacional Autónoma de México. Tlalnepantla, Estado de México, México. 198 pp.

Chávez-Mejía, M. C. 1993. Etnobotánica de San Jerónimo Bonchete. Una comunidad campesina de San Felipe del Progreso, México. Tesis de licenciatura. Facultad de Ciencias Agrícolas, Universidad Autónoma del Estado de México. Toluca, Estado de México, México. 139 pp.

Díaz-Roldán, A. V. 2013. Diagnósis de la vegetación y flora del Parque Nacional Nevado de Toluca (Estado de México, México) y propuesta para su gestoría. Tesis de licenciatura. Facultad de Estudios Superiores Iztacala, Universidad Nacional Autónoma de México. Tlalnepantla, Estado de México, México. 71 pp.

Dorantes-Hernández, F. I. y V. Piña-Dorantes. 2013. Estudio del ecotono del bosque de encino y tropical caducifolio en la cuenca del río Tlapala, Coatepec Harinas, Estado de México, México. Tesis de licenciatura. Facultad de Estudios Superiores Iztacala, Universidad Nacional Autónoma de México. Tlalnepantla, Estado de México, México. 107 pp.

Espejo-Serna, M. A. 2003. Sistemática del complejo de especies de Tillandsia plumosa Baker (Tillandsioideae: Bromeliaceae). 
Apéndice 1: Continuación.

Tesis de doctorado. Universidad Autónoma Metropolitana. México, D.F., México. 113 pp.

Esquivel-Romero, E. A. 1989. Contribución al conocimiento de la flora medicinal del poblado de Santa Catarina del Monte, municipio de Texcoco, Estado de México. Tesis de licenciatura. Facultad de Estudios Superiores Iztacala, Universidad Nacional Autónoma de México. Tlalnepantla, Estado de México, México. $132 \mathrm{pp}$.

Estrada-Hernández, M. V. 2011. Flora acuática de Almoloya de Juárez, Ixtlahuaca, Toluca y Villa Victoria, Estado de México. Tesis de licenciatura. Facultad de Ciencias Agrícolas, Universidad Autónoma del Estado de México. Toluca, Estado de México, México. 113 pp.

Estrada-Martínez, E. 1996. Etnobotánica forestal en Santa Isabel Chalma, Amecameca, México. Tesis de maestría. Colegio de Postgraduados, Campus Montecillo. Texcoco, Estado de México, México. 270 pp.

Figueroa-Solano, M. E. 2000. Uso agroecológico, actual y potencial de especies arbóreas en una selva baja caducifolia perturbada del suroeste del Estado de México. Tesis de maestría. Colegio de Postgraduados, Campus Montecillo. Texcoco, Estado de México, México. 120 pp.

Flores-Consuelo, A. M. S. 2004. Etnobotánica de un bosque de pinoencino en La Era, San Pablo Tlalchichilpa, municipio de San Felipe del Progreso, Estado de México. Tesis de licenciatura. Facultad de Ciencias, Universidad Autónoma del Estado de México. Toluca, Estado de México, México. 135 pp.

Flores-Cruz, M. 1998. Flora genérica de la familia Bromeliaceae en el Estado de México. Tesis de maestría. Facultad de Ciencias, Universidad Nacional Autónoma de México. México, D.F., México. 188 pp.

Fragoso-Ramírez, R. 1990. Estudio florístico en la parte alta de la Sierra de Zacualpan, Estado de México. Tesis de licenciatura. Facultad de Estudios Superiores Iztacala, Universidad Nacional Autónoma de México. Tlalnepantla, Estado de México, México. 69 pp.

Fuentes-Mayo, V. 2012. Atributos demográficos y biología reproductiva de Coryphantha cornifera y Stenocactus anfractuosus con fines de conservación. Tesis de doctorado. Colegio de Postgraduados, Campus Montecillo. Texcoco, Estado de México, México. 99 pp.
Galicia-Miranda, M. V. 1992. Listado florístico del Estado de México y regiones circundantes (de los estados de Hidalgo, Querétaro y Distrito Federal) basado en las colecciones de Eizi Matuda. Tesis de licenciatura. Facultad de Ciencias, Universidad Nacional Autónoma de México. México, D.F., México. 207 pp.

García-del Valle, L. G. 1999. Evaluación forestal del parque nacional Nevado de Toluca, Estado de México. Tesis de maestría. Facultad de Ciencias, Universidad Nacional Autónoma de México. México, D.F., México. 89 pp.

García-Gil, A. M. 1987. El género Passiflora en el Estado de México. Tesis de licenciatura. Facultad de Estudios Superiores Iztacala, Universidad Nacional Autónoma de México. Tlalnepantla, Estado de México, México. 126 pp.

García-Guerrero, N. A. 2007. La familia Amaranthaceae en el Estado de México. Tesis de licenciatura. Facultad de Estudios Superiores Iztacala, Universidad Nacional Autónoma de México. Tlalnepantla, Estado de México, México. 79 pp.

García-Hernández, A. R. 1999. Inventario florístico del municipio de Jilotepec, Estado de México. Tesis de licenciatura. Facultad de Estudios Superiores Iztacala, Universidad Nacional Autónoma de México. Tlalnepantla, Estado de México, México. 105 pp.

García-Mendoza, A. J. 1999. Revisión taxonómica del género Furcraea (Agavaceae) en México y Guatemala, proyecto No. H111. Instituto de Biología, Universidad Nacional Autónoma de México. Informe final Sistema Nacional de Información sobre Biodiversidad (SNIB)-Comisión Nacional para el Conocimiento y Uso de la Biodiversidad (CONABIO). México, D.F., México.

García-Ruiz, I. 1983. Contribución al conocimiento de los árboles y arbustos de Bejucos, Estado de México. Tesis de licenciatura. Facultad de Estudios Superiores Iztacala, Universidad Nacional Autónoma de México. Tlalnepantla, Estado de México, México. $105 \mathrm{pp}$

Gómez-Martínez, A. 2008. Monografía del género Tagetes spp. Tesis de licenciatura. Facultad de Ciencias Agrícolas, Universidad Autónoma del Estado de México. Toluca, Estado de México, México. $151 \mathrm{pp}$.

González-Arias, V. 2015. Listado florístico de las Magnoliophyta del predio hacienda Tejalpa, municipio de Zinacantepec, Estado de México. Tesis de licenciatura. Facultad de Ciencias Agrícolas, 
Apéndice 1: Continuación.

Universidad Autónoma del Estado de México. Toluca, Estado de México, México. 38 pp.

González-Martínez, F. y S. Rodríguez-Zaragoza. 1987. Estado actual de la vegetación de un área de bosque de encino-pino en Villa del Carbón, Estado de México. Tesis de licenciatura. Facultad de Estudios Superiores Iztacala, Universidad Nacional Autónoma de México. Tlalnepantla, Estado de México, México. 91 pp.

González-Olivares, H. R. 2012. Orquídeas del parque universitario José Mariano Mociño, Temascaltepec, México, 2011. Tesis de licenciatura. Facultad de Ciencias Agrícolas, Universidad Autónoma del Estado de México. Toluca, Estado de México, México. $62 \mathrm{pp}$.

Guizar-Nolazco, E. 1983. Estudio ecológico florístico de la vegetación del municipio de Tejupilco, Estado de México. Tesis de licenciatura, Universidad Autónoma Chapingo. Texcoco, Estado de México, México. 146 pp.

Hernández-Reyes, M. 1985. Levantamiento florístico de plantas fanerogámicas en el rancho de la Facultad de Estudios Superiores (FES), Cuautitlán, Estado de México. Tesis de licenciatura. Facultad de Ciencias, Universidad Nacional Autónoma de México. México, D.F., México.

Jiménez-Cruz, A. 2012. Estudio florístico del parque estatal Sierra de Tepotzotlán, Estado de México. Tesis de licenciatura. Facultad de Estudios Superiores Iztacala, Universidad Nacional Autónoma de México. Tlalnepantla, Estado de México, México. 103 pp.

Jiménez-Flores, J. 1994. Plantas medicinales de San Juan Tepecoculco, municipio de Atlautla de Victoria, Estado de México. Tesis de licenciatura. Facultad de Estudios Superiores Zaragoza, Universidad Nacional Autónoma de México. México, D.F., México. 320 pp.

Juárez-Medina, A. K. 2013. Diversidad alfa y beta de tres bosques mesófilos de montaña de México ubicados en diferentes provincias biogeográficas. Tesis de maestría. Facultad de Ciencias, Universidad Nacional Autónoma de México. México, D.F., México. 58 pp.

Limon-Boyce, A. E. 1980. Vegetación y lluvia de polen en el cerro Tetepetl, Estado de México. Tesis de licenciatura. Facultad de Ciencias, Universidad Nacional Autónoma de México. México, D.F., México. 89 pp.
López-Cruz, X. 2013. Estudio ecológico de los bosques de encino con Quercus urbanii Trel. y Quercus crassipes H. \& B. (Fagaceae) en dos Áreas Naturales Protegidas del Estado de México. Tesis de licenciatura. Facultad de Estudios Superiores Iztacala, Universidad Nacional Autónoma de México. Tlalnepantla, Estado de México, México. 203 pp.

López-Zamora, O. 2014. Composición florística y estructura de la vegetación en dos cañadas de recarga hídrica en Malinalco, Estado de México. Tesis de licenciatura. Facultad de Ciencias, Universidad Autónoma del Estado de México. Toluca, Estado de México, México. 121 pp.

Lozano-Mascarua, G. I. 1996. Plantas medicinales utilizadas por los mazahuas del municipio de San Felipe del Progreso, Estado de México. Tesis de licenciatura. Facultad de Ciencias, Universidad Nacional Autónoma de México. México, D.F., México. 271 pp.

Luna-Céspedes, R. 2014. Flora de las barrancas de Tonatico, Estado de México, México. Tesis de licenciatura. Facultad de Estudios Superiores Iztacala, Universidad Nacional Autónoma de México. Tlalnepantla, Estado de México, México. 111 pp.

Maldonado-Garcés, D. 2013. Flora útil y catálogo ilustrado de las especies encontradas en la comunidad de Coatepec Harinas, Estado de México. Tesis de licenciatura. Facultad de Estudios Superiores Iztacala, Universidad Nacional Autónoma de México. Tlalnepantla, Estado de México, México. 258 pp.

Manrique-Forceck, E. A. 1988. Gramineae del distrito de Temascaltepec, Estado de México. Tesis de licenciatura. Facultad de Ciencias, Universidad Nacional Autónoma de México. México, D.F., México. 89 pp.

Martínez-De La Cruz, I. 2010. La flora y vegetación ruderal de Malinalco, Estado de México. Tesis de maestría. Colegio de Postgraduados, Campus Montecillo. Texcoco, Estado de México, México. 149 pp.

Matías-González, B. 2009. Estudio etnobotánico de un grupo mazahua de Ixtlahuaca, Estado de México. Tesis de licenciatura. Facultad de Ciencias, Universidad Autónoma del Estado de México. Toluca, Estado de México, México. 108 pp.

Mayorga-Ramos, A. y J. L. Vasquez-Nieto. 2000. Impacto del tlacolol sobre la diversidad florística en el sur del municipio de Zumpahuacán, Estado de México. Tesis de licenciatura. 
Apéndice 1: Continuación.

Universidad Autónoma Chapingo. Toluca, Estado de México, México. 117 pp.

Mejía-Canales, A. 2014. Composición florística y estructura de la vegetación de un campo de cultivo abandonado en El Cerrillo Piedras Blancas, Toluca, Estado de México. Tesis de licenciatura. Facultad de Ciencias, Universidad Autónoma del Estado de México. Toluca, Estado de México, México. 102 pp.

Miranda-Jiménez, M. E. y M. A. González-Ortiz. 1993. Estudio de la vegetación y florística de la mesa basáltica de Holotepec, distrito de Tenango del Valle, Estado de México. Tesis de licenciatura. Facultad de Estudios Superiores Iztacala, Universidad Nacional Autónoma de México. Tlalnepantla, Estado de México, México. $74 \mathrm{pp}$.

Miranda-Uribe, A. 2008. Variación florística en los huertos familiares de San Nicolás, Malinalco, Estado de México. Tesis de licenciatura. Facultad de Ciencias, Universidad Autónoma del Estado de México. Toluca, Estado de México, México. 70 pp.

Moreno-Ortega, J. 2003. Uso potencial de la flora existente en el ejido de Zepayautla, Tenancingo, México. Tesis de licenciatura. Facultad de Ciencias Agrícolas, Universidad Autónoma del Estado de México. Toluca, Estado de México, México. 297 pp.

Muñoz-Flores, A. M. 2009. Diagnóstico ambiental de la sub-cuenca de Otumba, Estado de México. Tesis de licenciatura. Facultad de Estudios Superiores Iztacala, Universidad Nacional Autónoma de México. Tlalnepantla, Estado de México, México. 91 pp.

Nava-Bernal, J. H. 2008. Las orquídeas del municipio de Ocuilan de Arteaga, Estado de México. Tesis de licenciatura. Centro Universitario UAEM Tenancingo, Universidad Autónoma del Estado de México. Tenancingo, Estado de México. México. 126 pp.

Núñez-Reynoso, J. E. 1990. Estudio florístico de la vertiente oriental de la Sierra de Alcaparrosa, en el Estado de México. Tesis de licenciatura. Facultad de Estudios Superiores Iztacala, Universidad Nacional Autónoma de México. Tlalnepantla, Estado de México, México. 122 pp.

Ochoa-Kato, K. Y. 2013. Flora y estructura de los bosques en "Rancho Cerro Gordo" municipio de Valle de Bravo, Estado de México. Tesis de licenciatura. Facultad de Estudios Superiores Iztacala, Universidad Nacional Autónoma de México. Tlalnepantla, Estado de México, México. 88 pp.
Olivares-Castañeda, O. 2005. Catálogo florístico de especies vegetales de interés medicinal y aromático del parque botánico las orquídeas en Temascaltepec, Estado de México. Tesis de licenciatura. Facultad de Ciencias Agrícolas, Universidad Autónoma del Estado de México. Toluca, Estado de México, México. 407 pp.

Otento-Hernández, L. y Y. Ávalos-Martínez. 1993. Contribución al conocimiento de la familia Cactaceae del noroeste del Estado de México. Tesis de licenciatura. Facultad de Estudios Superiores Iztacala, Universidad Nacional Autónoma de México. Tlalnepantla, Estado de México, México. 140 pp.

Palacios-Carrillo, E. 1996. Localización, colecta y determinación taxonómica de especies vegetales silvestres de interés ornamental en los municipios de Temascaltepec y Zinacantepec. Tesis de licenciatura. Facultad de Ciencias Agrícolas, Universidad Autónoma del Estado de México. Toluca, Estado de México, México. 201 pp.

Palafox-Bárcenas, P. 1999. Análisis de vegetación en el cerro Tetzcutzinco, San Nicolás Tlaminca, Texcoco, México. Tesis de licenciatura. Universidad Autónoma Chapingo. Texcoco, Estado de México, México. 140 pp.

Ramírez-Cruz, B. 2009. Contribución al estudio ecológico de dos especies de encino Quercus obtusata Humb. \& Bonpl. y Quercus castanea Née, en dos localidades del Estado de México. Tesis de licenciatura. Facultad de Estudios Superiores Iztacala, Universidad Nacional Autónoma de México. Tlalnepantla, Estado de México, México. 91 pp.

Ramos-Ventura, L. J. 1999. Estudio de la flora y la vegetación acuáticas vasculares de la cuenca alta del río Lerma, en el Estado de México. Tesis de maestría. Facultad de Ciencias, Universidad Nacional Autónoma de México. México, D.F., México. 146 pp.

Rimarachín-Cabrera, I. 1997. Género y biodiversidad en una comunidad Otomí del Estado de México. Tesis de maestría. Colegio de Postgraduados, Campus Montecillo. Texcoco, Estado de México, México. 212 pp.

Rivas-Manzano, I. V. y H. Vibrans. 2000. Composición florística de la Unidad de San Cayetano, Villa de Allende, asociada a sitios con distinto tipo de perturbación. Informe Académico de Investigación. Clave: 1383/99. Facultad de Ciencias, 
Apéndice 1: Continuación.

Universidad Autónoma del Estado de México. Toluca, Estado de México, México.

Rodríguez-Barquet, L. E. y P. V. Rodríguez-Sánchez. 2013. Estudio del bosque templado húmedo en la cuenca del río Las Flores, Coatepec Harinas, Estado de México. Tesis de licenciatura. Facultad de Estudios Superiores Iztacala, Universidad Nacional Autónoma de México. Tlalnepantla, Estado de México, México. 98 pp.

Rodríguez-Colorado, A. R. 2002. La familia Loranthaceae en el Estado de México. Tesis de licenciatura. Facultad de Estudios Superiores Iztacala, Universidad Nacional Autónoma de México. Tlalnepantla, Estado de México, México. 102 pp.

Rodríguez-Olvera, M. 2001. Inventario y evaluación sanitaria del arbolado en Fundación Xochitla A.C. Tepotzotlán, Estado de México. Tesis de licenciatura. Facultad de Estudios Superiores Iztacala, Universidad Nacional Autónoma de México. Tlalnepantla, Estado de México, México. 65 pp.

Romero-Romero, F. 2006. Diversidad biológica, remanentes de vegetación natural y conservación de la cañada de Encinillas en Polotitlán, Estado de México. Tesis de maestría. Facultad de Ciencias, Universidad Nacional Autónoma de México. México, D.F., México. 128 pp.

Sánchez-González, A. 2004. Análisis sinecológico, florístico y biogeográfico de la vegetación del norte de la Sierra Nevada, México. Tesis de doctorado. Colegio de Postgraduados, Campus Montecillo. Texcoco, Estado de México, México. 153 pp.

SEMARNAT. 2010. Inventarios florísticos y faunísticos de la cuenca alta del río Lerma. Plan maestro para la restauración ambiental de la cuenca alta del río Lerma. Gobierno del Estado de México, Universidad Autónoma Chapingo. Toluca, México. 214 pp.

Silva-Pérez, L. del C. 1998. Los bosques de coníferas del sur de la cuenca de México: fitosociología, diversidad y uso tradicional. Tesis de licenciatura. Facultad de Ciencias, Universidad Autónoma del Estado de México. Toluca, Estado de México, México. $61 \mathrm{pp}$.

Tapia-Robles, C. A. 1985. Estudio morfológico y taxonómico de la familia Orchidaceae en el municipio de Temascaltepec. Tesis de licenciatura. Facultad de Ciencias Agrícolas, Universidad Autónoma del Estado de México. Toluca, Estado de México, México. 155 pp.
Téllez-Valdés, O. 2009. Base de datos de las Fabaceae y Caesalpiniaceae (Sensu Cronquist) y Dioscoreaceae de México, proyecto DS001. Universidad Nacional Autónoma de México. Facultad de Estudios Superiores Iztacala. Informe final Sistema Nacional de Información sobre Biodiversidad (SNIB)-Comisión Nacional para el Conocimiento y Uso de la Biodiversidad (CONABIO). México, D.F., México.

Torres-Bahena, E. 1993. Contribución al conocimiento de las plantas medicinales en la localidad de Malinalco, Estado de México. Tesis de licenciatura. Escuela Nacional de Ciencias Biológicas, Instituto Politécnico Nacional. México, D.F., México. 86 pp.

Ugalde-Lezama, S. 2005. Diversidad y distribución vertical de aves en un bosque con diferentes grados de perturbación en Zoquiapan, Estado de México. Tesis de maestría. Colegio de Postgraduados, Campus Montecillo. Texcoco, Estado de México, México. 132 pp.

Valdez-Palma, D. 2003. Estudio florístico del Parque universitario las Orquídeas en Temascaltepec, Estado de México. Tesis de licenciatura. Facultad de Ciencias Agrícolas, Universidad Autónoma del Estado de México. Toluca, Estado de México, México. 216 pp.

Vega-Aviña, R. 1982. Manual de la flora de la estación experimental de enseñanza e investigación y servicios forestales Zoquiapan. Tesis de maestría. Colegio de Postgraduados, Campus Montecillo. Texcoco, Estado de México, México. 364 pp.

Vega-López, A. 2003. Ecología de Brosimum alicastrum Sw. en el sur del Estado de México. Tesis de maestría. Colegio de Postgraduados, Campus Montecillo. Texcoco, Estado de México, México. 181 pp.

Vega-Silva, V. M. 2005. Flora fanerogámica y propuesta ecoturística rural en la comunidad de San José Deguedó, municipio de Soyaniquilpan, Estado de México. Tesis de licenciatura. Facultad de Estudios Superiores Iztacala, Universidad Nacional Autónoma de México. Tlalnepantla, Estado de México, México. 177 pp.

Zepeda-Peña, N. A. 2008. Flora de San Pedro Arriba, Temoaya, Estado de México. Tesis de licenciatura. Facultad de Ciencias Agrícolas, Universidad Autónoma del Estado de México. Toluca, Estado de México, México. 250 pp. 
Apéndice 1: Continuación.

Zúñiga-Soto, S. N. 2006. Diagnóstico del estudio de la vegetación en el Parque Nacional Nevado de Toluca, Estado de México. Tesis de licenciatura. Facultad de Ciencias, Universidad Nacional Autónoma de México. México, D.F., México. 65 pp. 
Apéndice 2: Literatura publicada compilada en la base de datos de las angiospermas del Estado de México.

Aguilera-Gómez, L. I., I. V. Rivas-Manzano, I. Martínez-De La Cruz y C. J. Aguilar-Ortigoza. 2016. El bosque tropical subcaducifolio en dos cañadas de Tlatlaya, Estado de México. Polibotánica 41: 1-29. DOI: https://dx.doi.org/10.18387/polibotanica.41.1

Aguirre-León, E. 2009. Orquídeas. In: Ceballos, G., R. List, G. Garduño, R. López-Cano, M. J. Muñozcano, E. Collado y J. E. San Román (comps.). La diversidad biológica del Estado de México. Estudio de Estado. Gobierno del Estado de México. Toluca, México. Pp. 203-208.

Almeida-Leñero, L., M. Escamilla, J. Giménez de Azcárate, A. González-Trápaga y A. M. Cleef. 2007. Vegetación alpina de los volcanes Popocatépetl, Iztaccíhuatl y Nevado de Toluca. In: Luna, I., J. J. Morrone y D. Espinosa (eds.). Biodiversidad de la Faja Volcánica Transmexicana. Universidad Nacional Autónoma de México y Comisión Nacional para el Conocimiento y Uso de la Biodiversidad. Cd. Mx., México. Pp. 179-198.

Álvarez-Lopeztello, J., I. V. Rivas-Manzano, L. I. Aguilera-Gómez y M. González-Ledesma. 2016. Diversidad y estructura de un pastizal en El Cerrillo, Piedras Blancas, Estado de México, México. Revista Mexicana de Biodiversidad 87(3): 980-989. DOI: https://dx.doi.org/10.1016/j.rmb.2016.06.006

Arreguín, M. de la L., L. Cabrera, R. Fernández, C. Orozco, B. Rodríguez y M. Yepez. 1997. Introducción a la flora del estado de Querétaro. Consejo de Ciencia y Tecnología del Estado de Querétaro. Querétaro, México. 361 pp.

Avilés-Nova,F.,L.M.Ríos-GarcíayC.A.Tapia-Robles.2012.Lasgramíneas silvestres del municipio de Temascaltepec, México: identificación, morfología, distribución y composición química. Universidad Autónoma del Estado de México. Toluca, México. 324 pp.

Balslev, H. y R. Duno de Stefano. 2015. La familia Juncaceae en México. Acta Botanica Mexicana 111: 61-164. DOI: https:// dx.doi.org/10.21829/abm111.2015.182

Barrie, F. R. 2003. Seven new species and one new variety of Valeriana (Valerianaceae) from Mexico. Acta Botanica Mexicana 62: 3164. DOI: https://dx.doi.org/10.21829/abm62.2003.914

Borhidi, A. 2006. Rubiáceas de México. Akadémiai Kiadó. Budapest, Hungría. 512 pp.

Calderón de Rzedowski, G. y J. Rzedowski. 2005. Flora Fanerogámica del Valle de México. Instituto de Ecología, A.C. y Comisión
Nacional para el Conocimiento y Uso de la Biodiversidad. Pátzcuaro, México. 1406 pp.

Castañeda-Rojas, A., I. S. Franco-Martínez y A. García-Mendoza. 2005. Manfreda galvaniae (Agavaceae), especie nueva de México, con nota sobre la ubicación taxonómica de M. malinaltenangensis Matuda y su lectotipificación. Acta Botanica Mexicana 72: 65-76. DOI: https://dx.doi. org/10.21829/abm72.2005.1002

Chimal-Hernández, A., M. González-Ibarra y C. Hernández-Díaz. 2013. La flora vascular del parque estatal El Faro, Tlalmanalco de Velázquez, Estado de México. Universidad Autónoma Metropolitana. Cd. Mx., México. 176 pp.

Dávila, P. 2009. Gramíneas. In: Ceballos, G., R. List, G. Garduño, R. López-Cano, M. J. Muñozcano, E. Collado y J. E. San Román (comps.). La diversidad biológica del Estado de México. Estudio de Estado. Gobierno del Estado de México. Toluca, México. Pp. 209-212.

Espejo-Serna, A. 2002. Viridantha, un género nuevo de Bromeliaceae (Tillandsioideae) endémico de México. Acta Botanica Mexicana 60: 25-35. DOI: https://dx.doi.org/10.21829/abm60.2002.901

Espejo-Serna, A. 2012. El endemismo en las Liliopsida mexicanas. Acta Botanica Mexicana 100: 195-257. DOI: https://dx.doi. org/10.21829/abm100.2012.36

Espejo-Serna, A. y A. R. López-Ferrari. 1993. Las monocotiledóneas mexicanas, una sinopsis florística 1. Lista de referencia, parte II. Anthericaceae, Araceae, Arecaceae, Asparagaceae, Asphodelaceae y Asteliaceae. Consejo Nacional de la Flora de México, Universidad Autónoma Metropolitana y Comisión Nacional para el Conocimiento y Uso de la Biodiversidad. México, D.F., México. 70 pp

Espejo-Serna, A. y A. R. López-Ferrari. 1994. Las monocotiledóneas mexicanas, una sinopsis florística 1. Lista de referencia, parte III. Bromeliaceae, Burmanniaceae, Calochortaceae y Cannaceae. Consejo Nacional de la Flora de México, Universidad Autónoma Metropolitana y Comisión Nacional para el Conocimiento y Uso de la Biodiversidad. México, D.F., México. 73 pp.

Espejo-Serna, A. y A. R. López-Ferrari. 2004. Checklist of Mexican Bromeliaceae with notes on species distribution and levels of endemism. Selbyana 25(1): 33-86. 
Apéndice 2: Continuación.

Fernández-Nava, R., Ma. de la L. Arreguín-Sánchez, C. RodríguezJiménez y A. Rodríguez-Jiménez. 1998. Listado florístico de la cuenca del río Balsas, México. Polibotánica 9: 1-151.

García-Rubio, L. A., O. Vargas-Ponce, F. de J. Ramírez-Mireles, G. Munguía-Lino, C. A. Corona-Oceguera y T. Cruz-Hernández. 2015. Distribución geográfica de Hylocereus (Cactaceae) en México. Botanical Sciences 93(4): 921-939. DOI: https://dx.doi. $\operatorname{org} / 10.17129 /$ botsci.282

Gaytán-Ávila, C., H. Vibrans, H. Navarro-Garza y M. JiménezVelázquez. 2001. Manejo de huertos familiares periurbanos de San Miguel Tlaixpan, Texcoco, Estado de México. Boletín de la Sociedad Botánica de México 69: 39-62.

González-Gutiérrez, M. 1989. El género Potamogeton (Potamogetonaceae) en México. Acta Botanica Mexicana 6: 1-43. DOI: https://dx.doi.org/10.21829/abm6.1989.576

González-Rocha, E. y R. Cerros-Tlatilpa. 2015. La familia Apocynaceae (Apocynoideae y Rauvolfioideae) en el estado de Morelos, México. Acta Botanica Mexicana 110: 21-70. DOI: https://dx.doi.org/10.21829/abm110.2015.191

Guzmán, U., S. Arias y P. Dávila. 2003. Catálogo de cactáceas mexicanas. Universidad Nacional Autónoma de México y Comisión Nacional para el Conocimiento y Uso de la Biodiversidad. México, D.F., México. 315 pp.

Halbinger, F. y M. Soto. 1997. Laelias de México. Orquídea (Méx.) 15: $1-160$.

Hernández-Cruz, M. J., S. D. Koch-Olt, M. T. P. Pulido-Salas, M. Luna-Cavazos y E. García-Villanueva. 2016. Estudio florístico del cerro Metecatl, del complejo montañoso Tetzcutzingo, Texcoco, Estado de México, México. Botanical Sciences 94(2): 377-392. DOI: https://dx.doi.org/10.17129/botsci.240

Huidobro-Salas, M. E. y E. Aguirre-León. 2009. Bromeliáceas. In: Ceballos, G., R. List, G. Garduño, R. López-Cano, M. J. Muñozcano, E. Collado y J. E. San Román (comps.). La diversidad biológica del Estado de México. Estudio de Estado. Gobierno del Estado de México. Toluca, México. Pp. 199-202.

Ibarra-Manríquez, G., F. J. Rendón-Sandoval, G. Cornejo-Tenorio y P. Carrillo-Reyes. 2015. Lianas of Mexico. Botanical Sciences 93(3): 365-417. DOI: https://dx.doi.org/10.17129/botsci.123
Lira, R., J. L. Villaseñor y E. Ortiz. 2002. A proposal for the conservation of the family Cucurbitaceae in México. Biodiversity and Conservation 11(10): 1699-1720. DOI: https:// doi.org/10.1023/a:1020303905416

Lira-Saade, R. 2009. Cucurbitáceas. In: Ceballos, G., R. List, G. Garduño, R. López-Cano, M. J. Muñozcano, E. Collado y J. E. San Román (comps.). La diversidad biológica del Estado de México. Estudio de Estado. Gobierno del Estado de México. Toluca, México. Pp. 213-217.

López-Patiño, E. J., D. R. Szeszko, J. Rescala-Pérez y A. S. Beltrán-Retis. 2012. The flora of the Tenancingo-MalinalcoZumpahuacán protected natural area, state of Mexico, Mexico. Harvard Papers in Botany 17(1): 65-167. DOI: https://doi. org/10.3100/025.017.0113

López-Pérez, Y., J. D. Tejero-Díez, A. N. Torres-Díaz e I. Luna-Vega. 2011. Flora del bosque mesófilo de montaña y vegetación adyacente en Avándaro, Valle de Bravo, Estado de México, México. Boletín de la Sociedad Botánica de México 88: 35-53. DOI: https://dx.doi.org/10.17129/botsci.304

López-Sandoval, J. A., E. A. Hernández-Arana, E. J. Morales-Rosales, A. Domínguez-López y L. M. Vázquez-García. 2007. Lista florística del Campus Universitario El Cerrillo de la Universidad Autónoma del Estado de México. Ciencias Agrícolas Informa 16: 55-65.

López-Sandoval, J. A., J. I. Valdez-Mercado, L. M. Vázquez-García, E. J. Morales-Rosales y A. Domínguez-López. 2008. Listado florístico preliminar del Cerro de Jocotitlán, Estado de México. Ciencias Agrícolas Informa 17: 28-42.

López-Sandoval, J. A., S. D. Koch, L. M. Vázquez-García, G. Munguía-Lino y E. J. Morales-Rosales. 2010. Estudio florístico de la parte central de la barranca Nenetzingo, municipio de Ixtapan de la Sal, Estado de México. Polibotánica 30: 9-33.

Luna-Vega, I., L. Almeida-Leñero y J. Llorente-Bousquets. 1989. Florística y aspectos fitogeográficos del bosque mesófilo de montaña de las Cañadas de Ocuilan, estados de Morelos y México. Anales del Instituto de Biología, Universidad Nacional Autónoma de México, Serie Botánica 59(1): 63-87.

Martínez, M. y E. Matuda. 1979. Flora del Estado de México. Tomos I, II y III. Edición fascimilar de los fascículos publicados en los 
Apéndice 2: Continuación.

años de 1953 a 1972. Biblioteca Enciclopédica del Estado de México. México, D.F., México. 1547 pp.

Martínez, M., O. Vargas-Ponce, A. Rodríguez, F. Chiang y S. Ocegueda. 2017. Solanaceae family in Mexico. Botanical Sciences 95(1): 131-145. DOI: https://dx.doi.org/10.17129/botsci.658

Martínez-De La Cruz, I., H. Vibrans, L. Lozada-Pérez, A. RomeroManzanares, L. I. Aguilera-Gómez e I. V. Rivas-Manzano. 2015. Plantas ruderales del área urbana de Malinalco, Estado de México, México. Botanical Sciences 93(4): 907-919. DOI: https://dx.doi.org/10.17129/botsci.213

Medina-Lemus, J. G. y J. D. Tejero-Díez. 2006. Flora y vegetación del parque estatal Atizapán-Valle Escondido, Estado de México, México. Polibotánica 21: 1-43.

Mejía-Saulés, Ma. T. y P. Dávila-Aranda. 1992. Gramíneas útiles de México. Instituto de Biología, Universidad Nacional Autónoma de México, Serie Cuadernos de Biología 16: 1-298.

Melchert, T. E. 2010. Bidens L. In: Turner, B. L. (ed.). The Comps of Mexico. A systematic account of the family Asteraceae (Chapter 10: subfamily Coreopsideae). Phytologia Memoirs 15: 3-56.

Méndez-Larios, I. y J. L. Villaseñor. 2001. La familia Scrophulariaceae en México: Diversidad y distribución. Boletín de la Sociedad Botánica de México 69: 101-121.

Mendoza-Castelán, G. y J. García-Pérez. 2000. Uso terapéutico de las plantas medicinales del Cerro Tetzcutzingo, municipio de Texcoco: manual. Departamento de Fitotecnia, Universidad Autónoma Chapingo, Programa de Investigación en Historia (PROIH)-Centro de Investigaciones Económicas, Sociales y Tecnológicas de la Agroindustria y la Agricultura Mundial (CIESTAAM). Chapingo, México. 180 pp.

Meyrán-García, J. y L. López-Chávez. 2003. Las crasuláceas de México. Sociedad Mexicana de Cactología. México, D.F., México. 234 pp.

Morales-Briones, D. F. 2016. Lachemilla mexiquense (Rosaceae), a new species from Mexico. PhytoKeys 62: 25-32. DOI: https:// doi.org/10.3897/phytokeys.62.7953

Morán, R. y J. Meyrán, 1987. Sedum tehuaztlense, una nueva especie del Estado de México. Cactáceas y Suculentas Mexicanas 32: 3-8.

Mora-Olivo, A., J. L. Villaseñor y M. Martínez. 2013. Las plantas vasculares acuáticas estrictas y su conservación en México.
Acta Botanica Mexicana 103: 27-63. DOI: https://dx.doi. org/10.21829/abm103.2013.50

Munguía-Lino, G., G. Vargas-Amado, L. M. Vázquez-García y A. Rodríguez. 2015. Riqueza y distribución geográfica de la tribu Tigridieae (Iridaceae) en Norteamérica. Revista Mexicana de Biodiversidad 86(1): 80-98. DOI: https://dx.doi.org/10.7550/ rmb.44083

Obieta, Ma. C. y J. Sarukhán. 1981. Estructura y composición de la vegetación herbácea de un bosque uniespecífico de Pinus hartwegii. Boletín de la Sociedad Botánica de México 41: 75-125.

Ocampo, G. y A. L. Medellín. 2004. Catálogo de ejemplares tipo del herbario IEB (México). Acta Botanica Mexicana 67: 1-41. DOI: https://dx.doi.org/10.21829/abm67.2004.971

Olvera, M. 1996. El género Utricularia (Lentibulariaceae) en México. Anales del Instituto de Biología, Universidad Nacional Autónoma de México, Serie Botánica 67(2): 347-384.

Pulido, M. T. P. y S. D. Koch. 1992. Guía ilustrada de las plantas del Cerro Tetzcutzingo, especies comunes en el Valle de México. Instituto de Biología, Universidad Nacional Autónoma de México, Serie Cuadernos de Biología 17: 1-245.

Ramírez-Rodríguez, R. y O. Téllez-Valdés. 1992. Las Dioscóreas (Dioscoreaceae) del estado de Morelos, México. Anales del Instituto de Biología, Universidad Nacional Autónoma de México, Serie Botánica 63(1): 67-99.

Redonda-Martínez, R. 2017. Diversidad y distribución de la tribu Vernonieae (Asteraceae) en México. Acta Botanica Mexicana 119: 115-138. DOI: https://dx.doi.org/10.21829/ abm119.2017.1235

Reyes-Santiago, J. 2009. Cactos. In: Ceballos, G., R. List, G. Garduño, R. López-Cano, M. J. Muñozcano, E. Collado y J. E. San Román (comps.). La diversidad biológica del Estado de México. Estudio de Estado. Gobierno del Estado de México. Toluca, México. Pp. 219-228.

Rodríguez, A. y L. Ortiz-Catedral. 2002. Nuevas localidades de tigridias mexicanas: Tigridia duranguense, T. ehrenbergii subsp. ehrenbergii y T. hallbergii subsp. lloydii. Ibugana 10(1-2): 61-66.

Rodríguez, A. y L. Ortiz-Catedral. 2003. Tigridia gracielae (Tigridieae: Iridaceae), a new species from México. Acta Botanica Mexicana 64: 31-36. DOI: https://dx.doi.org/10.21829/abm64.2003.926 
Apéndice 2: Continuación.

Rodríguez-Jiménez, C., R. Fernández-Nava, Ma. de la L. ArreguínSánchez y A. Rodríguez-Jiménez. 2005. Plantas vasculares endémicas de la cuenca del río Balsas. Polibotánica 20: 7399.

Rodríguez-Rivera, I. S. y S. Romero-Rangel. 2007. Arquitectura foliar de diez especies de encino (Quercus, Fagaceae) de México. Acta Botanica Mexicana 81: 9-34. DOI: https://dx.doi.org/10.21829/ abm81.2007.1049

Rojas-Zenteno, E. C., M. Orozco-Villa, S. Romero-Rangel y R. Montoya-Ayala. 2016. Vegetación y flora del municipio de Temascaltepec, Estado de México, México. Polibotánica 42: 4389. DOI: https://dx.doi.org/10.18387/polibotanica.42.3

Romero-Rangel, S. y E. C. Rojas-Zenteno. 1991. Estudio florístico de la región de Huehuetoca, Estado de México. Acta Botanica Mexicana 14: 33-57. DOI: https://dx.doi.org/10.21829/ abm14.1991.613

Romero-Rangel, S., E. C. Rojas Zenteno y L. E. Rubio-Licona. 2015. Encinos de México (Quercus, Fagaceae) 100 especies. Universidad Nacional Autónoma de México, Facultad de Estudios Superiores Iztacala. Cd. Mx., México. 304 pp.

Romero-Rangel, S., E. C. Rojas-Zenteno y M. de L. Aguilar-Enríquez. 2002. El género Quercus (Fagaceae) en el Estado de México. Annals of the Missouri Botanical Garden 89(4): 551-593.

Rubio-Licona, L. E., S. Romero-Rangel y E. C. Rojas-Zenteno. 2011. Estructura y composición florística de dos comunidades con presencia de Quercus (Fagaceae) en el Estado de México. Revista Chapingo Serie Forestales y del Ambiente 17(1): 77-90. DOI: http://dx.doi.org/10.5154/r.rchscfa.2010.03.014

Rzedowski, J. y G. Calderón de Rzedowski. 2010. Una especie nueva de Perymenium (Compositae, Heliantheae) del Estado de México (México). Acta Botanica Mexicana 91: 21-25. DOI: https://dx.doi.org/10.21829/abm91.2010.288

Sánchez-González, A. y L. López-Mata. 2003. Clasificación y ordenación de la vegetación del norte de la Sierra Nevada, a lo largo de un gradiente altitudinal. Anales del Instituto de Biología, Universidad Nacional Autónoma de México, Serie Botánica 74(1): 47-71.

Sánchez-González, A., L. López-Mata y H. Vibrans. 2006. Composición y patrones de distribución geográfica de la flora del bosque de oyamel del Cerro Tláloc, México. Boletín de la Sociedad Botánica de México 79: 67-78.

Sánchez-Ken, J. G. 2010. Two new species of Paspalum (Paniceae: Panicoideae: Poaceae), a preliminary checklist of the genus in Mexico, and the identity of P. crinitum. Revista Mexicana de Biodiversidad 81: 629-647.

Sánchez-Ken, J. G. 2012. A synopsis of Digitaria (Paniceae, Panicoideae, Poaceae) in Mexico, including the new species Digitaria michoacanensis. Acta Botanica Mexicana 101: 127149. DOI: https://dx.doi.org/10.21829/abm101.2012.28

Sánchez-Ken, J. G., G. de los A. Zita-Padilla y M. Mendoza-Cruz. 2012. Catálogo de las gramíneas malezas nativas e introducidas de México. Consejo Nacional Consultivo Fitosanitario (CONACOFI). México, D.F., México. 433 pp.

Sandoval-Moran, L. S. 2016. Flora nativa de Valle de Bravo para el diseño de paisaje. Publicación independiente ISBN 978-607009984-7. Cd. Mx., México. 278 pp.

SEMARNAT. 2009. Las orquídeas del Estado de México. Secretaría del Medio Ambiente, Gobierno del Estado de México. Toluca, México. 240 pp.

Solano, E. y A. García-Mendoza. 2013. Neotipificación y reconocimiento de Polianthes geminiflora (Lex.) Rose (Agavaceae). Acta Botanica Mexicana 104: 1-18. DOI: https:// dx.doi.org/10.21829/abm104.2013.54

Sorensen, P. D. 1969. Revision of the genus Dahlia (Compositae, Heliantheae-Coreopsidinae). Rhodora 71: 309-416.

Szeszko, D. R. 2011. La orquideoflora mexiquense. Secretaría de Educación del Gobierno del Estado de México. Toluca, México. $362 \mathrm{pp}$.

Tejero-Díez, J. D. y M. E. Castilla Hernández. 1999. Un método para el estudio del entorno ecológico en las primeras civilizaciones en Mesoamérica. Teotihuacán, un ejemplo. In: González-Jácome, A. y S. del Amo-Rodríguez (comps.). Agricultura y sociedad en México; diversidad, enfoques, estudios de caso. Plaza Valdés, Gestión de Ecosistemas A.C. y Universidad Iberoamericana. México, D.F., México. Pp. 33-60.

Téllez-Valdés, O. 2009. Leguminosas. In: Ceballos, G., R. List, G. Garduño, R. López-Cano, M. J. Muñozcano, E. Collado y J. E. San Román (comps.). La diversidad biológica del Estado de 
Apéndice 2: Continuación.

México. Estudio de Estado. Gobierno del Estado de México. Toluca, México. Pp. 195-198.

Torres-Soria, P. 2001. Flora fanerogámica de la zona arqueológica de Teotihuacán, Estado de México. Polibotánica 12: 57-83.

Torres-Zúñiga, Ma. M. y J. D. Tejero. 1998. Flora y vegetación de la Sierra de Sultepec, Estado de México. Anales del Instituto de Biología, Universidad Nacional Autónoma de México, Serie Botánica 69(2): 135-174.

Trejo-Díaz, C. C. y J. D. Tejero-Díez. 2017. Flora de plantas vasculares en la Sierra de las Ánimas, Chapa de Mota, Estado de México, México. Polibotánica 43: 1-35. DOI: https://dx.doi. $\operatorname{org} / 10.18387 /$ polibotanica.43.1

Valencia, S. 2004. Diversidad del género Quercus (Fagaceae) en México. Boletín de la Sociedad Botánica de México 75: 33-53. Vargas-Amado, G., A. Castro-Castro, M. Harker, J. L. Villaseñor, E. Ortiz y A. Rodríguez. 2013. Distribución geográfica y riqueza del género Cosmos (Asteraceae: Coreopsideae). Revista Mexicana de Biodiversidad 84: 536-555. DOI: https://dx.doi. $\operatorname{org} / 10.7550 / \mathrm{rmb} .31481$

Vázquez-Alonso, M. T., R. Bye, L. López-Mata, Ma. T. P. PulidoSalas, E. McClung de Tapia y S. D. Koch. 2014. Etnobotánica de la cultura Teotihuacana. Botanical Sciences 92(4): 563-574. DOI: https://dx.doi.org/10.17129/botsci.118

Vázquez-García, J. A., D. Jimeno, R. Cuevas, M. Cházaro y M. A. Muñiz-Castro. 2013. Echeveria yalmanantlanensis (Crassulaceae): A new species from Cerro Grande, Sierra de Manantlán, western Mexico. Brittonia 65(3): 273-279. DOI: https://doi.org/10.1007/s12228-012-9274-9

Vázquez-García, L. M. y J. A. López-Sandoval. 2010. Plantas con potencial ornamental del Estado de México. Universidad Autónoma del Estado de México. Toluca, México. 207 pp.
Vieyra-Odilon, L. y H. Vibrans. 2001. Weeds as crops: the value of maize field weeds in the valley of Toluca, Mexico. Economic Botany 55(3): 426-443. DOI: https://doi.org/10.1007/ bf02866564

Villarreal-Quintanilla, J, A., A. E. Estrada-Castillón y D. J. Rodríguez. 2009. El género Gentiana (Gentianaceae) en México. Polibotánica 27: 1-16.

Villaseñor, J. L. y E. Ortiz. 2007. La familia Asteraceae. In: Luna, I., J. J. Morrone y D. Espinosa (eds.). Biodiversidad de la Faja Volcánica Transmexicana. Universidad Nacional Autónoma de México y Comisión Nacional para el Conocimiento y Uso de la Biodiversidad. Cd. Mx., México. Pp. 289-310.

Villaseñor, J. L. y F. J. Espinosa. 1998. Catálogo de Malezas de México. Universidad Nacional Autónoma de México y Fondo de Cultura Económica. México, D.F., México. 448 pp.

White-Olascoaga, L., J. I. Juan-Pérez, C. Chávez-Mejía y J. G. Gutiérrez-Castillo. 2013. Flora medicinal en San Nicolás, Municipio de Malinalco, Estado de México. Polibotánica 35: 173-206.

Zepeda-Gómez, C. y E. Velázquez-Montes. 1999. El bosque tropical caducifolio de la vertiente sur de la Sierra de Nanchititla, Estado de México: la composición y la afinidad geográfica de su flora. Acta Botanica Mexicana 46: 29-55. DOI: https://dx.doi. org/10.21829/abm46.1999.815

Zepeda-Gómez, C., A. Lot-Helgueras, X. A. Nemiga y D. MadrigalUribe. 2012. Florística y diversidad de las Ciénegas del río Lerma Estado de México, México. Acta Botanica Mexicana 98: 23-49. DOI: https://dx.doi.org/10.21829/abm98.2012.1139 
Apéndice 3: Especies excluidas de la lista de angiospermas para el Estado de México. Fueron documentadas para el estado por diferentes autores; sin embargo, puede tratarse de determinaciones erróneas y no cuentan con un ejemplar de herbario que respalde su presencia en el estado.

\section{MAGNOLIIDES}

Piperaceae

Peperomia angustata Kunth

Peperomia cordovana C. DC.

Piper palmeri C. DC.

\section{MONOCOTILEDÓNEAS}

Alismataceae

Sagittaria lancifolia L.

\section{Araceae}

Spathiphyllum blandum Schott

\section{Asparagaceae}

Agave funkiana K. Koch \& C.D. Bouché

Furcraea quicheensis Trel.

\section{Bromeliaceae}

Hechtia glabra Brandegee

Hechtia macrophylla Greenm.

Hechtia stenopetala Klotzsch

Pitcairnia tuerckheimii Donn. Sm.

Tillandsia baileyi Rose ex Small

Tillandsia califani Rauh

Tillandsia filifolia Schltdl. \& Cham.

Tillandsia leiboldiana Schltdl.

Tillandsia multicaulis Steud.

Tillandsia paraisoensis Ehlers

Tillandsia rodrigueziana $\mathrm{Mez}$

Tillandsia utriculata $\mathrm{L}$.

Tillandsia viridiflora (Beer) Baker

Viridantha mauryana (L.B. Sm.) Espejo

\section{Commelinaceae}

Callisia multiflora (M. Martens \& Galeotti) Standl.

Commelina jaliscana Matuda

\section{Cyperaceae}

Cyperus polystachyos Rottb.

\section{Dioscoreaceae}

Dioscorea orizabensis Uline

\section{Iridaceae}

Sisyrinchium abietum McVaugh

\section{Juncaceae}

Juncus dudleyi Wiegand

Juncus ensifolius Wikstr.

Juncus nodosus L.

Juncus saximontanus A. Nelson

Luzula vulcanica Liebm.

\section{Marantaceae}

Maranta gibba Sm.

\section{Melanthiaceae}

Schoenocaulon caricifolium (Schltdl.) A. Gray

Schoenocaulon obtusum Brinker

\section{Orchidaceae}

Acianthera hartwegiifolia (H. Wendl. \& Kraenzl.) R. Solano \& Soto Arenas

Anathallis minutalis (Lindl.) Pridgeon \& M.W. Chase

Aulosepalum nelsonii (Greenm.) Garay

Bletia amabilis $\mathrm{C}$. Schweinf.

Bletia ensifolia L.O. Williams

Bulbophyllum cirrhosum L.O. Williams

Clowesia glaucoglossa (Rchb. f.) Dodson

Cranichis cochleata Dressler

Cuitlauzina pulchella (Bateman ex Lindl.) Dressler \& N.H. Williams Deiregyne chartacea (L.O. Williams) Garay

Dinema polybulbon (Sw.) Lindl.

Encyclia papillosa (Bateman) Aguirre-Olav.

Epidendrum caroli Schltr.

Epidendrum examinis S. Rosillo

Epidendrum lowilliamsii García-Cruz

Epidendrum mocinnoi Hágsater

Epidendrum tortipetalum Scheeren

Goodyera brachyceras (A. Rich. \& Galeotti) Garay \& G.A. Romero

Habenaria agapitae R. González \& Reynoso

Habenaria ibarrae R. González

Habenaria ixtlanensis E.W. Greenw.

Habenaria pinzonii R. González \& Cuev.-Fig.

Habenaria ruizii R. González

Habenaria rzedowskii R. González

Malaxis brachystachys (Rchb. f.) Kuntze

Malaxis contrerasii R. González

Malaxis pringlei (S. Watson) Ames

Malaxis rodrigueziana $\mathrm{R}$. González

Malaxis tequilensis R. González, Lizb. Hern. \& E. Ramírez 
Apéndice 3: Continuación.

Malaxis urbana E.W. Greenw.

Mormodes ramirezii S. Rosillo

Pelexia funckiana Schltr.

Platanthera sparsiflora (S. Watson) Schltr.

Prosthechea karwinskii (Mart.) J.M.H. Shaw

Prosthechea trulla (Rchb. f.) W.E. Higgins

Sarcoglottis assurgens (Rchb. f.) Schltr.

Stelis guerrerensis Soto Arenas \& R. Solano

Stelis immersa (Linden \& Rchb. f.) Pridgeon \& M.W. Chase

Triphora yucatanensis Ames

\section{Poaceae}

Andropogon leucostachyus Kunth

Axonopus poiophyllus Chase

Bromus marginatus Nees ex Steud.

Calamagrostis recta (Kunth) Trin. ex Steud.

Cottea pappophoroides Kunth

Digitaria obtusa Swallen

Elymus trachycaulus (Link) Gould ex Shinners

Ichnanthus pallens (Sw.) Munro ex Benth.

Muhlenbergia elongata Scribn. ex Beal

Muhlenbergia grandis Vasey

Muhlenbergia longiglumis Vasey

Muhlenbergia tenuissima (J. Presl) Kunth

Panicum polygonatum Schrad.

Rhipidocladum racemiflorum (Steud.) McClure

Setaria leucopila (Scribn. \& Merr.) K. Schum.

Sporobolus airoides (Torr.) Torr.

\section{EUDICOTILEDÓNEAS}

\section{Acanthaceae}

Bravaisia berlandieriana (Nees) T.F. Daniel

\section{Aizoaceae}

Sesuvium verrucosum Raf.

\section{Anacardiaceae}

Comocladia macrophylla (Hook. \& Arn.) L. Riley

Rhus mollis Jacq.

\section{Apocynaceae}

Mandevilla hirsuta (Rich.) K. Schum.

Marsdenia gualanensis Donn. Sm.

Matelea reticulata (Engelm. ex A. Gray) Woodson

Thenardia chiapensis J.K. Williams

\author{
Asteraceae \\ Acourtia platyphylla (A. Gray) Reveal \& R.M. King \\ Ageratina geminata (McVaugh) R.M. King \& H. Rob. \\ Ageratina havanensis (Kunth) R.M. King \& H. Rob. \\ Ageratina triniona (McVaugh) R.M. King \& H. Rob. \\ Ageratum microcephalum Hemsl. \\ Alloispermum scabrifolium (Hook. \& Arn.) H. Rob. \\ Alomia callosa (S. Watson) B.L. Rob. \\ Baltimora recta $\mathrm{L}$. \\ Bidens bicolor Greenm. \\ Bidens ferulifolia (Jacq.) DC. \\ Bidens heterosperma A. Gray \\ Brickellia jaliscensis McVaugh \\ Critoniopsis obtusa (Gleason) H. Rob. \\ Dahlia purpusii Brandegee \\ Erigeron divergens Torr. \& A. Gray \\ Gamochaeta standleyi (Steyerm.) G.L. Nesom \\ Hymenostephium guatemalense (B.L. Rob. \& Greenm.) S.F. Blake \\ Iostephane madrensis (S. Watson) Strother \\ Lagascea angustifolia DC. \\ Lasianthaea palmeri (Greenm.) K.M. Becker \\ Melampodium appendiculatum B.L. Rob. \\ Pentacalia parasitica (Hemsl.) H. Rob. \& Cuatrec. \\ Perityle turneri A.M. Powell \\ Perymenium alticola McVaugh \\ Perymenium ghiesbreghtii B.L. Rob. \& Greenm. \\ Perymenium subsquarrosum B.L. Rob. \& Greenm. \\ Pinaropappus spathulatus Brandegee \\ Psacalium eriocarpum (S.F. Blake) S.F. Blake \\ Pseudogynoxys haenkei (DC.) Cabrera \\ Ratibida columnifera (Nutt.) Wooton \& Standl. \\ Roldana kerberi (Greenm.) H. Rob. \& Brettell \\ Roldana robinsoniana (Greenm.) H. Rob. \& Brettell \\ Roldana subpeltata (Sch. Bip.) H. Rob. \& Brettell \\ Stevia berlandieri A. Gray \\ Stevia ephemera Grashoff \\ Stevia tephrophylla S.F. Blake \\ Stevia zephyrantha Grashoff \\ Steviopsis dryophila (B.L. Rob.) B.L. Turner \\ Telanthophora grandifolia (Less.) H. Rob. \& Brettell \\ Telanthophora standleyi (Greenm.) H. Rob. \& Brettell \\ Trixis haenkei Sch. Bip. \\ Trixis hintoniorum B.L. Turner \\ Verbesina persicifolia DC. \\ Verbesina platyptera Sch. Bip. ex Klatt
}


Apéndice 3: Continuación.

\section{Begoniaceae}

Begonia barkeri Knowles \& Westc.

Begonia sandtii Houghton ex Ziesenh.

Begonia tapatia Burt-Utley \& McVaugh

Begonia uruapensis Sessé \& Moc.

\section{Berberidaceae}

Berberis trifolia (Schltdl. \& Cham.) Schult. \& Schult. f.

\section{Bignoniaceae}

Handroanthus guayacan (Seem.) S.O. Grose

\section{Boraginaceae}

Hackelia leonotis I.M. Johnst.

Heliotropium angustifolium Torr.

Heliotropium convolvulaceum (Nutt.) A. Gray

Heliotropium macrostachyum (DC.) Hemsl.

Nama sericea Willd. ex Roem. \& Schult.

Phacelia secunda J.F. Gmel.

\section{Brassicaceae}

Draba myosotidioides Hemsl.

Pennellia lasiocalycina (O.E. Schulz) Rollins

\section{Burseraceae}

Bursera biflora (Rose) Standl.

Bursera microphylla A. Gray

Bursera odorata Brandegee

\section{Cactaceae}

Cephalocereus senilis (Haw.) Pfeiff.

Coryphantha echinus (Engelm.) Britton \& Rose

Disocactus cinnabarinus (Eichlam ex Weing.) Barthlott

Mammillaria carnea Zucc. ex Pfeiff.

Mammillaria sempervivi DC.

Opuntia cantabrigiensis Lynch

Pilosocereus purpusii (Britton \& Rose) Byles \& G.D. Rowley

Protium copal (Schltdl. \& Cham.) Engl.

Stenocereus fricii Sánchez-Mej.

\section{Campanulaceae}

Lobelia subnuda Benth.

\section{Caryophyllaceae}

Cerastium arvense $\mathrm{L}$.

\section{Celastraceae}

Wimmeria microphylla Radlk.

\section{Cleomaceae}

Andinocleome pilosa (Benth.) Iltis \& Cochrane

Cleome parvisepala Heilborn

\section{Combretaceae}

Combretum decandrum Jacq.

\section{Convolvulaceae}

Cuscuta costaricensis Yunck.

Cuscuta jalapensis Schltdl.

Cuscuta mexicana Yunck.

Cuscuta woodsonii Yunck.

Ipomoea decemcornuta O'Donell

Ipomoea lozanii Painter

Ipomoea plummerae A. Gray

Ipomoea sescossiana Baill.

\section{Crassulaceae}

Echeveria agavoides Lem.

Echeveria crenulata Rose

Echeveria harmsii J.F. Macbr.

\section{Cucurbitaceae}

Sicyos kunthii Cogn.

\section{Ericaceae}

Vaccinium caespitosum Michx.

\section{Euphorbiaceae}

Acalypha macrostachyoides Müll. Arg.

Acalypha ocymoides Kunth

Cnidoscolus elasticus Lundell

Euphorbia succedanea L.C. Wheeler

Euphorbia xalapensis Kunth

Euphorbia xylopoda Greenm.

Mabea occidentalis Benth.

Tragia brevispica Engelm. \& A. Gray

\section{Fabaceae}

Aeschynomene ciliata Vogel

Aeschynomene compacta Rose

Aeschynomene palmeri Rose

Apoplanesia paniculata C. Presl 
Apéndice 3: Continuación.

Astragalus hartwegii Benth.

Astragalus hintonii Barneby

Bauhinia coulteri J.F. Macbr.

Brongniartia argentea Rydb.

Brongniartia proteranthera L.B. Sm. \& B.G. Schub.

Canavalia oxyphylla Standl. \& L.O. Williams

Cologania intermedia Kunth

Crotalaria mexicana Windler

Crotalaria purshii DC.

Dalea erythrorhiza Greenm.

Dalea greggii A. Gray

Dalea lasiathera A. Gray

Dalea leucosericea (Rydb.) Standl. \& Steyerm.

Dalea melantha S. Schauer

Desmodium cajanifolium (Kunth) DC.

Desmodium caripense (Kunth) G. Don

Desmodium cordistipulum Hemsl.

Desmodium leptoclados Hemsl.

Desmodium urarioides (S.F. Blake) B.G. Schub. \& McVaugh

Diphysa sennoides Benth.

Eysenhardtia texana Scheele

Harpalyce mexicana Rose

Indigofera palmeri $\mathrm{S}$. Watson

Leucaena lanceolata S. Watson

Lonchocarpus castilloi Standl.

Lonchocarpus hondurensis Benth.

Marina ghiesbreghtii Barneby

Marina neglecta (B.L. Rob.) Barneby

Mimosa brevispicata Britton

Mimosa minutifolia B.L. Rob. \& Greenm.

Mimosa monancistra Benth.

Mimosa rhododactyla B.L. Rob.

Mucuna pruriens (L.) DC.

Nissolia platycarpa Benth.

Nissolia wislizenii (A. Gray) A. Gray

Pediomelum palmeri (Ockendon) J.W. Grimes ex Gandhi

Phaseolus acutifolius A. Gray

Phaseolus nelsonii Maréchal, Mascherpa \& Stainier

Pterocarpus acapulcensis Rose

Rhynchosia macrocarpa Benth.

Senna quinquangulata (Rich.) H.S. Irwin \& Barneby

Tephrosia abbottiae C.E. Wood

Tephrosia langlassei Micheli

Tephrosia multifolia Rose

Tephrosia submontana (Rose) L. Riley

\section{Fagaceae}

Quercus opaca Trel.

\section{Gentianaceae}

Zeltnera stricta (Schiede) G. Mans.

\section{Gesneriaceae}

Achimenes candida Lindl.

Achimenes misera Lindl.

Amalophyllon parviflorum (A. Br. \& Bouché) Boggan, L.E. Skog \& Roalson

\section{Hypericaceae}

Hypericum paniculatum Lam.

\section{Krameriaceae}

Krameria lanceolata Torr.

\section{Lamiaceae}

Persea schiedeana Nees

Salvia rubiginosa Benth.

Salvia teresae Fernald

Salvia veronicifolia A. Gray ex S. Watson

Salvia villosa Fernald

\section{Loganiaceae}

Spigelia chiapensis K.R. Gould

\section{Loranthaceae}

Struthanthus cassythoides Millsp. ex Standl.

\section{Lythraceae}

Cuphea elliptica Koehne

Cuphea infundibulum Koehne

Cuphea nitidula Kunth

\section{Malpighiaceae}

Callaeum johnsonii W.R. Anderson

Stigmaphyllon lindenianum A. Juss.

\author{
Malvaceae \\ Anoda albiflora Fryxell \\ Ayenia magna L. \\ Heliocarpus mexicanus (Turcz.) Sprague \\ Heliocarpus palmeri S. Watson \\ Heliocarpus parvimontis Gual
}


Apéndice 3: Continuación.

Hibiscus lavateroides Moric. ex Ser.

Malvella lepidota (A. Gray) Fryxell

Melochia lupulina Sw.

Melochia spicata (L.) Fryxell

Pavonia firmiflora Schery

Sida jussiaeana DC.

Thespesia populnea (L.) Sol. ex Corrêa

Triumfetta medusae W.W. Thomas \& McVaugh

Waltheria acuminata Rose

\section{Melastomataceae}

Heterocentron elegans (Schltdl.) Kuntze

Miconia alpestris Cogn.

Monochaetum alpestre Naudin

Tibouchina rufipilis (Schltdl.) Cogn.

\section{Meliaceae}

Guarea grandifolia DC.

Trichilia glabra L.

Trichilia minutiflora Standl.

\section{Moraceae}

Dorstenia excentrica Moric.

\section{Myrtaceae}

Eugenia uxpanapensis P.E. Sánchez \& L.M. Ortega

\section{Nyctaginaceae}

Boerhavia ciliata Brandegee

\section{Oleaceae}

Fraxinus americana $\mathrm{L}$.

Fraxinus pennsylvanica Marshall

\section{Onagraceae}

Lopezia grandiflora Zucc.

Oenothera biennis L.

\section{Passifloraceae}

Passiflora juliana J.M. MacDougal

Passiflora mcvaughiana J.M. MacDougal

Passiflora pilosa Ruiz \& Pav. ex DC.

Passiflora podadenia Killip

\section{Phyllanthaceae}

Phyllanthus evanescens Brandegee

\section{Picramniaceae}

Picramnia polyantha (Benth.) Planch.

\section{Polygalaceae}

Polygala pedicellata S.F. Blake

Polygala vergrandis W.H. Lewis

\section{Primulaceae}

Ardisia paschalis Donn. Sm.

Parathesis leptopa Lundell

\section{Ranunculaceae}

Clematis haenkeana C. Presl

Ranunculus forreri Greene

Thalictrum fendleri Engelm. ex A. Gray

\section{Rhamnaceae}

Colubrina ehrenbergii Schltdl.

Rhamnus sharpii M.C. Johnston \& L.A. Johnston

\section{Rosaceae}

Agrimonia gryposepala Wallr.

Crataegus baroussana Eggl.

Holodiscus argenteus (L. f.) Maxim.

\section{Rubiaceae}

Bouvardia gracilipes B.L. Rob.

Chione venosa (Sw.) Urb.

Deppea pubescens Hemsl.

Didymaea mexicana Hook. f.

Galium iltisii Dempster

Spermacoce alata Aubl.

Spermacoce tetraquetra A. Rich.

\section{Rutaceae}

Casimiroa sapota Oerst.

\section{Salicaceae}

Casearia sylvestris Sw.

Populus mexicana Wesm. ex DC.

\section{Santalaceae}

Arceuthobium gillii Hawksw. \& Wiens

Phoradendron angustifolium (Kunth) Eichler

Phoradendron robustissimum Eichler 
Apéndice 3: Continuación.

\section{Sapindaceae}

Serjania insignis Radlk.

Thouinidium oblongum Radlk.

\section{Solanaceae}

Bouchetia arniatera B.L. Rob.

Cestrum alternifolium (Jacq.) O.E. Schulz Cestrum fasciculatum (Schltdl.) Miers

Cestrum glanduliferum Kerber ex Francey Cestrum racemosum Ruiz \& Pav.

Cestrum scandens Vahl

Jaltomata repandidentata (Dunal) Hunz.

Physalis ampla Waterf.

Physalis latiphysa Waterf.

Physalis leptophylla B.L. Rob. \& Greenm.

Solanum bicorne Dunal

Solanum grayi Rose

Solanum leucandrum Whalen

\section{Thymelaeaceae}

Daphnopsis mollis (Schltdl. \& Cham.) Standl.

\section{Urticaceae}

Myriocarpa bifurca Liebm.

Pilea cadierei Gagnep. \& Guillaumin

Pilea ecboliophylla Donn. Sm.

Urera elata (Sw.) Griseb.

Urtica spirealis Blume

\section{Verbenaceae}

Lippia nutans B.L. Rob. \& Greenm.

Lippia queretarensis Kunth

\section{Violaceae}

Viola flagelliformis Hemsl.

\section{Zygophyllaceae}

Kallstroemia hirsutissima Vail ex Small 
Apéndice 4: Angiospermas nativas documentadas en la literatura para el Estado de México. Con un asterisco $(*)$ se indican las especies endémicas de México y con doble asterisco (**) las endémicas del Estado de México.

\section{MAGNOLIIDES}

\author{
Annonaceae \\ *Annona globiflora Schltdl. \\ Annona macroprophyllata Donn. Sm. \\ Annona reticulata $\mathrm{L}$.

\section{Aristolochiaceae} \\ *Aristolochia brevipes Benth. \\ *Aristolochia foetida Kunth \\ *Aristolochia styloglossa Pfeifer \\ *Aristolochia taliscana Hook. \& Arn.
}

\section{Cabombaceae}

Cabomba palaeformis Fassett

\section{Chloranthaceae}

Hedyosmum mexicanum C. Cordem.

\section{Hernandiaceae}

Gyrocarpus jatrophifolius Domin

\section{Lauraceae}

*Cinnamomum pachypodum (Nees) Kosterm.

Litsea glaucescens Kunth

Nectandra salicifolia (Kunth) Nees

Persea americana Mill.

*Persea hintonii C.K. Allen

\section{Nymphaeaceae}

*Nymphaea gracilis Zucc.

Nymphaea mexicana Zucc.

Nymphaea odorata Aiton

\section{Piperaceae}

Peperomia asarifolia Schltdl. \& Cham.

Peperomia bracteata A.W. Hill

*Peperomia cavispicata G. Mathieu

*Peperomia gracillima $\mathrm{S}$. Watson

Peperomia hintonii Yunck.

Peperomia hispidula (Sw.) A. Dietr.

Peperomia humilis A. Dietr.

Peperomia lanceolatopeltata C. DC.

Peperomia leptophylla Miq.

Peperomia pedicellata Dahlst.
Peperomia quadrifolia (L.) Kunth

Peperomia tetraphylla (G. Forst.) Hook. \& Arn.

Piper amalago L.

Piper auritum Kunth

Piper hispidum Sw.

*Piper leucophyllum (Miq.) C. DC.

Piper sanctum (Miq.) Schltdl. ex C. DC.

Piper umbellatum L.

\section{Saururaceae}

Anemopsis californica (Nutt.) Hook. \& Arn.

\section{MONOCOTILEDÓNEAS}

\section{Alismataceae}

Echinodorus andrieuxii (Hook. \& Arn.) Small

Sagittaria guayanensis Kunth

Sagittaria latifolia Willd.

Sagittaria longiloba Engelm. ex J.G. Sm.

*Sagittaria macrophylla Zucc.

Alstroemeriaceae

Bomarea acutifolia (Link \& Otto) Herb.

Bomarea edulis (Tussac) Herb.

\section{Amaryllidaceae}

Allium glandulosum Link \& Otto

Allium kunthii G. Don

*Allium stoloniferum Ownbey \& T.D. Jacobsen

**Allium telaponense Traub

*Habranthus concolor Lindl.

*Hymenocallis acutifolia (Herb.) Sweet

*Hymenocallis glauca (Zucc.) M. Roem.

*Hymenocallis harrisiana Herb.

Hymenocallis littoralis (Jacq.) Salisb.

Nothoscordum bivalve (L.) Britton

*Sprekelia formosissima (L.) Herb.

Zephyranthes brevipes (Baker) Standl.

Zephyranthes carinata Herb.

*Zephyranthes fosteri Traub

*Zephyranthes sessilis Herb.

Zephyranthes verecunda Herb.

\author{
Araceae \\ *Anthurium andicola Liebm. \\ Anthurium schlechtendalii Kunth
}


Apéndice 4: Continuación.

*Arisaema macrospathum Benth.

Lemna aequinoctialis Welw.

Lemna gibba L.

Lemna minuta Kunth

Lemna obscura (Austin) Daubs

Lemna trisulca $\mathrm{L}$.

Lemna valdiviana Phil.

*Philodendron basii Matuda

Philodendron hederaceum (Jacq.) Schott

Philodendron sagittifolium Liebm.

Pistia stratiotes L.

Spirodela polyrhiza (L.) Schleid.

Syngonium neglectum Schott

Wolffia brasiliensis Wedd.

Wolffia columbiana $\mathrm{H}$. Karst.

Wolffiella gladiata (Hegelm.) Hegelm.

Wolffiella lingulata (Hegelm.) Hegelm.

Wolffiella oblonga (Phil.) Hegelm.

*Xanthosoma mendozae Matuda

Xanthosoma mexicanum Liebm.

Xanthosoma wendlandii (Schott) Schott

\section{Arecaceae}

Acrocomia aculeata (Jacq.) Lodd. ex Mart.

Brahea dulcis (Kunth) Mart.

*Brahea pimo Becc.

Sabal mexicana Mart.

*Sabal pumos (Kunth) Burret

\section{Asparagaceae}

Agave americana $\mathrm{L}$.

*Agave angustiarum Trel.

Agave angustifolia Haw.

*Agave applanata Lem. ex Jacobi

*Agave atrovirens Karw. ex Salm-Dyck

*Agave attenuata Salm-Dyck

Agave dasylirioides Jacobi \& C.D. Bouché

*Agave filifera Salm-Dyck

* Agave horrida Lem. ex Jacobi

*Agave inaequidens $\mathrm{K}$. Koch

Agave lechuguilla Torr.

*Agave mapisaga Trel.

*Agave salmiana Otto ex Salm-Dyck

*Beschorneria wrightii Hook. f.

*Bessera elegans Schult. f.

*Dasylirion acrotrichum (Schiede) Zucc.
*Echeandia durangensis (Greenm.) Cruden

Echeandia echeandioides (Schltdl.) Cruden

Echeandia flavescens (Schult. \& Schult. f.) Cruden

*Echeandia flexuosa Greenm.

*Echeandia gracilis Cruden

*Echeandia hirticaulis Cruden

Echeandia longipedicellata Cruden

* Echeandia mexicana Cruden

*Echeandia nana (Baker) Cruden

*Echeandia paniculata Rose

Echeandia parviflora Baker

Echeandia reflexa (Cav.) Rose

*Furcraea parmentieri (Roezl ex Ortgies) García-Mend.

Furcraea pubescens Tod.

Maianthemum flexuosum (Bertol.) LaFrankie

Maianthemum paniculatum (M. Martens \& Galeotti) LaFrankie

Maianthemum scilloideum (M. Martens \& Galeotti) LaFrankie

**Manfreda galvaniae A. Castañeda, S. Franco \& García-Mend.

*Manfreda hauniensis (J.B. Petersen) Verh.-Will.

* Manfreda maculata (C. Mart.) Rose

**Manfreda malinaltenangensis Matuda

* Manfreda nanchititlensis Matuda

*Manfreda pringlei Rose

**Manfreda revoluta (Klotzsch) Rose

Manfreda scabra (Ortega) McVaugh

Milla biflora Cav.

*Milla magnifica H.E. Moore

*Nolina parviflora (Kunth) Hemsl.

*Polianthes geminiflora (Lex.) Rose

*Polianthes pringlei Rose

Polianthes tuberosa L.

*Yucca filifera Chabaud

Yucca guatemalensis Baker

\section{Bromeliaceae}

Bromelia hemisphaerica Lam.

Bromelia pinguin $\mathrm{L}$.

Catopsis nutans (Sw.) Griseb.

Catopsis paniculata E. Morren

Catopsis sessiliflora (Ruiz \& Pav.) Mez

*Hechtia caerulea (Matuda) L.B. Sm.

Hechtia glomerata Zucc.

*Hechtia hintoniana Burt-Utley, Utley \& García-Mend.

*Hechtia marnier-lapostollei L.B. Sm.

*Hechtia matudae L.B. Sm.

*Hechtia podantha Mez 
Apéndice 4: Continuación.

*Hechtia tillandsioides (André) L.B. Sm.

*Pitcairnia cylindrostachya L.B. Sm.

*Pitcairnia flexuosa L.B. Sm.

Pitcairnia heterophylla (Lindl.) Beer

*Pitcairnia hintoniana L.B. Sm.

*Pitcairnia karwinskyana Schult. \& Schult. f.

*Pitcairnia micropoda L.B. Sm.

*Pitcairnia palmeri S. Watson

*Pitcairnia pteropoda L.B. Sm.

*Tillandsia achyrostachys E. Morren ex Baker

*Tillandsia andrieuxii (Mez) L.B. Sm.

*Tillandsia bourgaei Baker

Tillandsia brachycaulos Schltdl.

Tillandsia capitata Griseb.

Tillandsia caput-medusae E. Morren

*Tillandsia chaetophylla Mez

*Tillandsia circinnatioides Matuda

Tillandsia compressa Bertero ex Schult. \& Schult. f.

*Tillandsia cossonii Baker

Tillandsia dasyliriifolia Baker

*Tillandsia dugesii Baker

*Tillandsia erubescens Schltdl.

Tillandsia fasciculata Sw.

*Tillandsia hintoniana L.B. Sm.

*Tillandsia hubertiana Matuda

Tillandsia ionantha Planch.

Tillandsia juncea (Ruiz \& Pav.) Poir.

*Tillandsia karwinskyana Schult. f.

*Tillandsia kirchhoffiana Wittm.

*Tillandsia langlasseana Mez

*Tillandsia macdougallii L.B. Sm.

Tillandsia makoyana Baker

*Tillandsia parryi Baker

Tillandsia polystachia (L.) L.

*Tillandsia prodigiosa (Lem.) Baker

Tillandsia recurvata (L.) L.

*Tillandsia roseospicata Matuda

Tillandsia schiedeana Steud.

*Tillandsia sessemocinoi López-Ferr., Espejo \& P. Blanco

Tillandsia streptophylla Scheidw. ex E. Morren

*Tillandsia sueae Ehlers

*Tillandsia superinsignis Matuda

*Tillandsia supermexicana Matuda

*Tillandsia thyrsigera E. Morren ex Baker

Tillandsia usneoides (L.) L.

*Tillandsia violacea Baker
**Tillandsia zacualpanensis Ehlers \& Wülfingh.

*Viridantha atroviridipetala (Matuda) Espejo

*Viridantha ignesiae (Mez) Espejo

*Viridantha lepidosepala (L.B. Sm.) Espejo

Viridantha plumosa (Baker) Espejo

*Viridantha tortilis (Klotzsch ex Baker) Espejo

\section{Ceratophyllaceae}

Ceratophyllum demersum $\mathrm{L}$.

\section{Commelinaceae}

*Callisia insignis C.B. Clarke

**Commelina bambusifolia Matuda

Commelina coelestis Willd.

*Commelina congestipantha López-Ferr., Espejo \& Ceja

Commelina dianthifolia Delile

Commelina diffusa Burm. f.

Commelina elliptica Kunth

Commelina erecta $\mathrm{L}$.

Commelina leiocarpa Benth.

Commelina orchioides Booth ex Lindl.

Commelina rufipes Seub.

*Commelina rzedowskii López-Ferr., Espejo \& Ceja

*Commelina scabra Benth.

Commelina texcocana Matuda

Commelina tuberosa $\mathrm{L}$.

Commelina virginica $\mathrm{L}$.

*Gibasis consobrina D.R. Hunt

Gibasis geniculata (Jacq.) Rohweder

*Gibasis karwinskyana (Schult. f.) Rohweder

*Gibasis linearis (Benth.) Rohweder

Gibasis pellucida (M. Martens \& Galeotti) D.R. Hunt

Gibasis pulchella (Kunth) Raf.

*Gibasis venustula (Kunth) D.R. Hunt

*Thyrsanthemum floribundum (M. Martens \& Galeotti) Pichon

*Thyrsanthemum goldianum D.R. Hunt

*Thyrsanthemum macrophyllum (Greenm.) Rohweder

Tinantia erecta (Jacq.) Schltdl.

Tinantia leiocalyx C.B. Clarke ex Donn. Sm.

Tinantia longipedunculata Standl. \& Steyerm.

Tinantia parviflora Rohweder

Tinantia standleyi Steyerm.

Tradescantia commelinoides Schult. \& Schult. f.

Tradescantia crassifolia Cav.

*Tradescantia llamasii Matuda

Tradescantia standleyi Steyerm. 
Apéndice 4: Continuación.

Tradescantia zebrina Bosse

*Tripogandra amplexans Handlos

Tripogandra amplexicaulis (Klotzsch ex C.B. Clarke) Woodson

Tripogandra angustifolia (B.L. Rob.) Woodson

Tripogandra disgrega (Kunth) Woodson

Tripogandra purpurascens (S. Schauer) Handlos

Tripogandra serrulata (Vahl) Handlos

Weldenia candida Schult. f.

\section{Cyperaceae}

*Abildgaardia mexicana (Palla) Kral

Amphiscirpus nevadensis (S. Watson) Oteng-Yeb.

Bolboschoenus maritimus (L.) Palla

Bulbostylis capillaris (L.) Kunth ex C.B. Clarke

Bulbostylis funckii (Steud.) C.B. Clarke

Bulbostylis juncoides (Vahl) Kük. ex Osten

Bulbostylis pubescens (J. Presl \& C. Presl) Svenson

Bulbostylis tenuifolia (Rudge) J.F. Macbr.

Carex anisostachys Liebm.

Carex athrostachya Olney

*Carex aztecica Mack

Carex boliviensis Van Heurck \& Müll. Arg.

Carex brunnipes Reznicek

Carex chordalis Liebm.

*Carex ciliaris Fernald

*Carex cochranei Reznicek

Carex cortesii Liebm.

*Carex coulteri Boott ex Hemsl.

*Carex curviculmis Reznicek

Carex echinata Murray

Carex geophila Mack.

Carex hermannii Cochrane

Carex humboldtiana Steud.

**Carex ixtapalucensis Reznicek

*Carex longicaulis Boeckeler

Carex longii Mack.

Carex lurida Wahlenb.

*Carex marianensis Stacey

Carex orizabae Liebm.

Carex peucophila Holm

Carex planostachys Kunze

Carex polystachya Sw. ex Wahlenb.

Carex praegracilis W. Boott

**Carex tolucensis (F.J. Herm.) Reznicek

*Carex tuberculata Liebm.

*Carex turbinata Liebm.
Carex vallicola Dewey

Carex xalapensis Kunth

Cyperus aggregatus (Willd.) Endl.

Cyperus amabilis Vahl

*Cyperus aschenbornianus Boeckeler

Cyperus bipartitus Torr.

*Cyperus calderoniae S. González

Cyperus canus J. Presl \& C. Presl

Cyperus compressus L.

Cyperus digitatus Roxb.

Cyperus esculentus $\mathrm{L}$.

Cyperus fendlerianus Boeckeler

Cyperus flavescens L.

Cyperus flavicomus Michx.

Cyperus hermaphroditus (Jacq.) Standl.

Cyperus ischnos Schltdl.

Cyperus laevigatus L.

Cyperus lanceolatus Poir.

Cyperus laxus Lam.

Cyperus manimae Kunth

Cyperus mutisii (Kunth) Andersson

Cyperus niger Ruiz \& Pav.

Cyperus ochraceus Vahl

Cyperus odoratus L.

Cyperus pallidicolor (Kük.) G.C. Tucker

*Cyperus pennellii O’Neill \& Ben. Ayers

Cyperus prolixus Kunth

Cyperus pycnostachyus (Kunth) Kunth

Cyperus reflexus Vahl

Cyperus sanguineo-ater Boeckeler

Cyperus semiochraceus Boeckeler

Cyperus seslerioides Kunth

Cyperus spectabilis Link

Cyperus sphaerolepis Boeckeler

Cyperus squarrosus L.

Cyperus surinamensis Rottb.

Cyperus tenerrimus J. Presl \& C. Presl

Cyperus tenuis Sw.

Cyperus virens Michx.

Eleocharis acicularis (L.) Roem. \& Schult.

Eleocharis bonariensis Nees

Eleocharis cancellata S. Watson

Eleocharis cellulosa Torr.

*Eleocharis densa Benth.

Eleocharis dombeyana Kunth

Eleocharis elegans (Kunth) Roem. \& Schult. 
Apéndice 4: Continuación.

Eleocharis filiculmis Kunth

Eleocharis geniculata (L.) Roem. \& Schult.

Eleocharis macrostachya Britton

Eleocharis minima Kunth

Eleocharis montana (Kunth) Roem. \& Schult.

Eleocharis montevidensis Kunth

Eleocharis mutata (L.) Roem. \& Schult.

*Fimbristylis argillicola Kral

Fimbristylis complanata (Retz.) Link

Fimbristylis dichotoma (L.) Vahl

Fimbristylis miliacea (L.) Vahl

Fuirena incompleta Nees

Fuirena simplex Vahl

*Karinia mexicana (C.B. Clarke ex Britton) Reznicek \& McVaugh

Kyllinga odorata Vahl

Kyllinga pumila Michx.

Lipocarpha micrantha (Vahl) G.C. Tucker

Rhynchospora aristata Boeckeler

Rhynchospora colorata (L.) H. Pfeiff.

Rhynchospora contracta (Nees) J. Raynal

Rhynchospora holoschoenoides (Rich.) Herter

Rhynchospora kunthii Nees ex Kunth

Rhynchospora nervosa (Vahl) Boeckeler

Schoenoplectus americanus (Pers.) Volkart ex Schinz \& R. Keller

Schoenoplectus californicus (C.A. Mey.) Soják

Schoenoplectus tabernaemontani (C.C. Gmel.) Palla

Scleria ciliata Michx.

Scleria reticularis Michx.

\section{Dioscoreaceae}

Dioscorea carpomaculata O. Téllez \& B.G. Schub.

Dioscorea convolvulacea Schltdl. \& Cham.

*Dioscorea galeottiana Kunth

*Dioscorea gallegosi Matuda

*Dioscorea guerrerensis R. Knuth

*Dioscorea hintonii R. Knuth

*Dioscorea jaliscana S. Watson

*Dioscorea liebmannii Uline

*Dioscorea longituba Uline

Dioscorea mexicana Scheidw.

*Dioscorea morelosana (Uline) Matuda

Dioscorea nelsonii Uline ex R. Knuth

*Dioscorea pantojensis R. Knuth

*Dioscorea platycolpota Uline ex B.L. Rob.

* Dioscorea plumifera B.L. Rob.

Dioscorea polygonoides Humb. \& Bonpl. ex Willd.
*Dioscorea pringlei B.L. Rob.

*Dioscorea remotiflora Kunth

*Dioscorea sanchez-colinii Matuda

Dioscorea subtomentosa Miranda

*Dioscorea temascaltepecensis R. Knuth

*Dioscorea tolucana (Matuda) Caddick \& Wilkin

*Dioscorea tubiperianthia Matuda

*Dioscorea ulinei Greenm. ex R. Knuth

*Dioscorea urceolata Uline

*Dioscorea uruapanensis Matuda

Eriocaulaceae

Eriocaulon benthamii Kunth

*Eriocaulon jaliscanum $\mathrm{S}$. Watson

Eriocaulon microcephalum Kunth

*Eriocaulon schiedeanum Körn.

\section{Hydrocharitaceae}

Limnobium laevigatum (Humb. \& Bonpl. ex Willd.) Heine

Najas guadalupensis (Spreng.) Magnus

Vallisneria americana Michx.

\section{Hypoxidaceae}

Hypoxis decumbens $\mathrm{L}$.

Hypoxis fibrata Brackett

Hypoxis mexicana Schult. \& Schult. f.

\section{Iridaceae}

Cipura campanulata Ravenna

Eleutherine latifolia (Stand1. \& L.O. Williams) Ravenna

Nemastylis tenuis (Herb.) S. Watson

Orthrosanthus exsertus (R.C. Foster) Ravenna

Sisyrinchium angustifolium Mill.

*Sisyrinchium angustissimum (B.L. Rob. \& Greenm.) Greenm. \& C.H. Thomps.

Sisyrinchium arizonicum Rothr.

*Sisyrinchium bracteatum Greenm.

Sisyrinchium cernuum (E.P. Bicknell) Kearney

Sisyrinchium convolutum Nocca

*Sisyrinchium conzattii Calderón \& Rzed.

*Sisyrinchium macrophyllum Greenm.

*Sisyrinchium quadrangulatum Klatt

Sisyrinchium scabrum Cham. \& Schltdl.

*Sisyrinchium schaffneri S. Watson

*Sisyrinchium serrulatum (E.P. Bicknell) Espejo \& López-Ferr. Sisyrinchium tenuifolium Humb. \& Bonpl. ex Willd. 
Apéndice 4: Continuación.

*Sisyrinchium tolucense Peyr.

*Tigridia alpestris Molseed

*Tigridia augusta Drapiez

*Tigridia ehrenbergii (Schltdl.) Molseed

*Tigridia fosteri Goldblatt

*Tigridia gracielae Aarón Rodr. \& Ortiz-Cat.

*Tigridia hallbergii Molseed

*Tigridia longispatha (Herb.) Goldblatt

*Tigridia matudae Molseed

Tigridia meleagris (Lindl.) G. Nicholson

*Tigridia mexicana Molseed

*Tigridia mortonii Molseed

*Tigridia multiflora (Herb.) Ravenna

Tigridia pavonia (L. f.) DC.

*Tigridia vanhouttei Roezl ex Van Houtte

\section{Juncaceae}

Juncus acuminatus Michx.

Juncus balticus Willd.

Juncus bufonius L.

Juncus dichotomus Elliott

Juncus ebracteatus E. Mey.

Juncus effusus L.

Juncus imbricatus Laharpe

Juncus liebmannii J.F. Macbr.

Juncus marginatus Rostk.

Juncus microcephalus Kunth

Juncus xiphioides E. Mey.

Luzula caricina E. Mey.

Luzula denticulata Liebm.

Luzula racemosa Desv.

\section{Juncaginaceae}

Triglochin mexicana Kunth

Triglochin scilloides (Poir.) Mering \& Kadereit

\section{Liliaceae}

*Calochortus barbatus (Kunth) J.H. Painter

Calochortus fuscus Schult. f.

Calochortus ghiesbreghtii S. Watson

*Calochortus pringlei B.L. Rob.

*Calochortus purpureus (Kunth) Baker

\section{Marantaceae}

Calathea allouia (Aubl.) Lindl.

*Calathea atropurpurea Matuda
*Calathea ovandensis Matuda

Maranta arundinacea L.

\section{Mayacaceae}

Mayaca fluviatilis Aubl.

\section{Melanthiaceae}

*Anticlea frigida (Schltdl. \& Cham.) Zomlefer \& Judd Anticlea virescens (Kunth) Rydb.

Schoenocaulon officinale (Schltdl. \& Cham.) A. Gray ex Benth.

*Schoenocaulon pringlei Greenm.

*Schoenocaulon rzedowskii Frame

*Schoenocaulon tenue Brinker

\section{Orchidaceae}

*Alamania punicea Lex.

Anathallis scariosa (Lex.) Pridgeon \& M.W. Chase

*Arpophyllum spicatum Lex.

Aulosepalum pyramidale (Lindl.) M.A. Dix \& M.W. Dix

*Aulosepalum tenuiflorum (Greenm.) Garay

Barkeria obovata (C. Presl) Christenson

*Barkeria scandens (Lex.) Dressler \& Halb.

Barkeria strophinx (Rchb. f.) Halb.

Barkeria uniflora (Lex.) Dressler \& Halb.

*Bletia adenocarpa Rchb. f.

Bletia campanulata Lex.

* Bletia coccinea Lex.

* Bletia concolor Dressler

Bletia gracilis Lodd.

*Bletia greenmaniana L.O. Williams

*Bletia lilacina A. Rich. \& Galeotti

*Bletia macristhmochila Greenm.

*Bletia neglecta Sosa

*Bletia parkinsonii Hook.

* Bletia punctata Lex.

Bletia purpurata A. Rich. \& Galeotti

Bletia purpurea (Lam.) DC.

Bletia reflexa Lindl.

*Bletia similis Dressler

*Bletia urbana Dressler

*Bulbophyllum nagelii L.O. Williams

*Catasetum laminatum Lindl.

*Clowesia thylaciochila (Lem.) Dodson

*Corallorhiza bulbosa A. Rich. \& Galeotti

Corallorhiza macrantha Schltr.

Corallorhiza maculata (Raf.) Raf. 
Apéndice 4: Continuación.

Corallorhiza odontorhiza (Willd.) Poir.

Corallorhiza striata Lindl.

Corallorhiza williamsii Correll

Corallorhiza wisteriana Conrad

*Cranichis subumbellata A. Rich. \& Galeotti

Cranichis sylvatica A. Rich. \& Galeotti

*Cuitlauzina dubia (S. Rosillo) Yáñez \& Soto Arenas ex Solano

*Cuitlauzina pendula Lex.

Cyclopogon elatus (Sw.) Schltr.

*Cyclopogon saccatus (A. Rich. \& Galeotti) Schltr.

Cypripedium irapeanum Lex.

Cyrtopodium macrobulbon (Lex.) G.A. Romero \& Carnevali

*Deiregyne albovaginata (C. Schweinf.) Garay

*Deiregyne densiflora (C. Schweinf.) Salazar \& Soto Arenas

Deiregyne eriophora (B.L. Rob. \& Greenm.) Garay

*Deiregyne pseudopyramidalis (L.O. Williams) Garay

*Deiregyne rhombilabia Garay

* Deiregyne tenorioi Soto Arenas \& Salazar

Dichaea squarrosa Lindl.

*Dichaea trichocarpa (Sw.) Lindl.

Dichromanthus aurantiacus (Lex.) Salazar \& Soto Arenas

Dichromanthus cinnabarinus (Lex.) Garay

Dichromanthus michuacanus (Lex.) Salazar \& Soto Arenas

*Domingoa kienastii (Rchb. f.) Dressler

Encyclia adenocarpa (Lex.) Schltr.

*Encyclia adenocaula (Lex.) Schltr.

*Encyclia meliosma (Rchb. f.) Schltr.

*Encyclia microbulbon (Hook.) Schltr.

*Epidendrum anisatum Lex.

Epidendrum ciliare L.

Epidendrum clowesii Bateman ex Lindl.

*Epidendrum cusii Hágsater

*Epidendrum dorsocarinatum Hágsater

Epidendrum eximium L.O. Williams

*Epidendrum lignosum Lex.

*Epidendrum matudae L.O. Williams

*Epidendrum miserum Lindl.

Epidendrum parkinsonianum Hook.

*Erycina hyalinobulbon (Lex.) N.H. Williams \& M.W. Chase

Funkiella hyemalis (A. Rich. \& Galeotti) Schltr.

Funkiella minutiflora (A. Rich. \& Galeotti) Salazar \& Soto Arenas

Funkiella parasitica (A. Rich. \& Galeotti) Salazar \& Soto Arenas

Funkiella rubrocallosa (B.L. Rob. \& Greenm.) Salazar \& Soto

Arenas

Galeoglossum tubulosum (Lind1.) Salazar \& Soto Arenas

Galeottiella sarcoglossa (A. Rich. \& Galeotti) Schltr.
Goodyera striata Rchb. f.

* Govenia capitata Lindl.

*Govenia dressleriana E.W. Greenw.

Govenia lagenophora Lindl.

Govenia liliacea (Lex.) Lindl.

Govenia purpusii Schltr.

Govenia superba (Lex.) Lindl.

Guarianthe aurantiaca (Bateman ex Lindl.) Dressler \& W.E. Higgins

*Habenaria calicis R. González

Habenaria clypeata Lindl.

Habenaria crassicornis Lindl.

Habenaria diffusa A. Rich. \& Galeotti

*Habenaria filifera $\mathrm{S}$. Watson

*Habenaria flexuosa Lindl.

*Habenaria gonzaleztamayoi García-Cruz, R. Jiménez \& L. Sánchez

Habenaria guadalajarana S. Watson

*Habenaria jaliscana S. Watson

*Habenaria macvaughiana R. González

Habenaria novemfida Lindl.

*Habenaria oreophila Greenm.

**Habenaria ortiziana R. González

Habenaria pringlei B.L. Rob.

Habenaria quinqueseta (Michx.) Eaton

*Habenaria rosulifolia Espejo \& López-Ferr.

*Habenaria rzedowskiana R. González

Habenaria strictissima Rchb. f.

Habenaria trifida Kunth

*Habenaria uncata R. Jiménez, L. Sánchez \& García-Cruz

*Habenaria virens A. Rich. \& Galeotti

*Habenaria zamudioana R. González

*Hagsatera brachycolumna (L.O. Williams) R. González

*Hexalectris brevicaulis L.O. Williams

Hexalectris grandiflora (A. Rich. \& Galeotti) L.O. Williams

*Hintonella mexicana Ames

*Homalopetalum pachyphyllum (L.O. Williams) Dressler

Homalopetalum pumilio (Rchb. f.) Schltr.

*Isochilus bracteatus (Lex.) Salazar \& Soto Arenas ex López-Ferr. \&

Espejo

Isochilus chiriquensis Schltr.

Isochilus linearis (Jacq.) R. Br.

*Jacquiniella cernua (Lindl.) Dressler

Jacquiniella leucomelana (Rchb. f.) Schltr.

*Kionophyton sawyeri (Standl. \& L.O. Williams) Garay

Kionophyton seminuda (Schltr.) Garay

*Laelia autumnalis (Lex.) Lindl.

Laelia rubescens Lindl. 
Apéndice 4: Continuación.

*Laelia speciosa (Kunth) Schltr.

Leochilus carinatus (Knowles \& Westc.) Lindl.

*Lepanthes nagelii Salazar \& Soto Arenas

Liparis cordiformis C. Schweinf.

*Liparis draculoides E.W. Greenw.

*Liparis greenwoodiana Espejo

Liparis vexillifera (Lex.) Cogn.

*Macroclinium lexarzanum (Hágsater \& R. González) Dodson

Malaxis abieticola Salazar \& Soto Arenas

*Malaxis amplexicolumna E.W. Greenw. \& R. González

Malaxis carnosa (Kunth) C. Schweinf.

Malaxis corymbosa (S. Watson) Kuntze

Malaxis elliptica A. Rich. \& Galeotti

*Malaxis espejoi R. González, Lizb. Hern. \& E. Ramírez

Malaxis fastigiata (Rchb. f.) Kuntze

Malaxis histionantha (Link, Klotzsch \& Otto) Garay \& Dunst.

Malaxis lepidota (Finet) Ames

*Malaxis lyonnetii Salazar

Malaxis maianthemifolia Schltdl. \& Cham.

*Malaxis myurus (Lindl.) Kuntze

*Malaxis reichei (Schltr.) Ames \& C. Schweinf.

*Malaxis ribana Espejo \& López-Ferr.

*Malaxis rosei Ames

*Malaxis rosilloi $\mathrm{R}$. González \& E.W. Greenw.

**Malaxis rzedowskiana $\mathrm{R}$. González

Malaxis soulei L.O. Williams

*Malaxis streptopetala (B.L. Rob. \& Greenm.) Ames

*Malaxis stricta L.O. Williams

Malaxis unifolia Michx.

*Malaxis zempoalensis López-Ferr. \& Espejo

Maxillaria cucullata Lindl.

*Maxillaria houtteana Rchb. f.

* Maxillaria lexarzana Soto Arenas \& F. Chiang

* Maxillaria mexicana J.T. Atwood

Maxillaria variabilis Bateman ex Lindl.

Mesadenus polyanthus (Rchb. f.) Schltr.

*Mesadenus tenuissimus (L.O. Williams) Garay

*Microepidendrum subulatifolium (A. Rich. \& Galeotti) W.E.

Higgins

*Mormodes aromatica Lindl.

*Nemaconia dressleriana (Soto Arenas) Van den Berg, Salazar \& Soto Arenas

*Oestlundia ligulata (La Llave \& Lex.) Soto Arenas

Oncidium brachyandrum Lindl.

Oncidium geertianum C. Morren

*Oncidium ghiesbreghtianum A. Rich. \& Galeotti
*Oncidium hastatum (Bateman) Lindl.

* Oncidium karwinskii (Lindl.) Lindl.

*Oncidium microstigma Rchb. f.

Oncidium reflexum Lindl.

Oncidium reichenheimii (Linden \& Rchb. f.) Garay \& Stacy

*Oncidium suave Lindl.

Oncidium tigrinum Lex.

*Oncidium unguiculatum Lindl.

*Physogyne sparsiflora (C. Schweinf.) Garay

Platanthera brevifolia (Greene) Kraenzl.

Platanthera limosa Lindl.

*Ponera exilis Dressler

Ponthieva ephippium Rchb. f.

Ponthieva formosa Schltr.

*Ponthieva hildae R. González \& R. Soltero

Ponthieva mexicana (A. Rich. \& Galeotti) Salazar

Ponthieva racemosa (Walter) C. Mohr

Ponthieva schaffneri (Rchb. f.) E.W. Greenw.

Prescottia stachyodes (Sw.) Lindl.

Prosthechea brassavolae (Rchb. f.) W.E. Higgins

Prosthechea chondylobulbon (A. Rich. \& Galeotti) W.E. Higgins

*Prosthechea citrina (Lex.) W.E. Higgins

*Prosthechea concolor (Lex.) W.E. Higgins

*Prosthechea cretacea (Dressler \& G.E. Pollard) W.E. Higgins

Prosthechea michuacana (Lex.) W.E. Higgins

*Prosthechea micropus (Rchb. f.) W.E. Higgins

*Prosthechea mulasii Soto Arenas \& L. Cerv.

*Prosthechea pastoris (Lex.) Espejo \& López-Ferr.

*Prosthechea pringlei (Rolfe) W.E. Higgins

*Prosthechea pterocarpa (Lindl.) W.E. Higgins

*Prosthechea punctulata (Rchb. f.) Soto Arenas \& Salazar

*Prosthechea squalida (Lex.) Soto Arenas \& Salazar

Prosthechea varicosa (Bateman ex Lindl.) W.E. Higgins

*Rhynchostele aptera (Lex.) Soto Arenas \& Salazar

*Rhynchostele cervantesii (Lex.) Soto Arenas \& Salazar

Rhynchostele maculata (Lex.) Soto Arenas \& Salazar

*Rossioglossum insleayi (Baker ex Lindl.) Garay \& G.C. Kenn.

Sacoila lanceolata (Aubl.) Garay

Sarcoglottis corymbosa Garay

Sarcoglottis schaffneri (Rchb. f.) Ames

Scaphyglottis sessilis (Rchb. f.) Foldats

*Schiedeella affinis (C. Schweinf.) Salazar

*Schiedeella crenulata (L.O. Williams) Espejo \& López-Ferr.

Schiedeella durangensis (Ames \& C. Schweinf.) Burns-Bal.

*Schiedeella garayana R. González

Schiedeella llaveana (Lindl.) Schltr. 
Apéndice 4: Continuación.

*Schiedeella tenella (L.O. Williams) Burns-Bal.

*Sobralia galeottiana A. Rich.

* Sotoa confusa (Garay) Salazar

Spiranthes graminea Lindl.

Spiranthes nebulorum Catling \& V.R. Catling

*Stanhopea hernandezii (Kunth) Schltr.

Stelis emarginata (Lindl.) Soto Arenas \& Solano

Stelis greenwoodii Soto Arenas \& Solano

*Stelis nigriflora (L.O. Williams) Pridgeon \& M.W. Chase

*Stelis oestlundiana (L.O. Williams) Pridgeon \& M.W. Chase

Stelis quadrifida (Lex.) Solano \& Soto Arenas

*Stelis retusa (Lex.) Pridgeon \& M.W. Chase

Stelis villosa (Knowles \& Westc.) Pridgeon \& M.W. Chase

*Stelis xerophila (Schltr.) Soto Arenas

*Svenkoeltzia congestiflora (L.O. Williams) Burns-Bal.

Tamayorkis ehrenbergii (Rchb. f.) R. González \& Szlach.

Trichocentrum brachyphyllum (Lindl.) R. Jiménez

Trichocentrum cebolleta (Jacq.) M.W. Chase \& N.H. Williams

*Trichocentrum pachyphyllum (Hook.) R. Jiménez \& Carnevali

Triphora trianthophora (Sw.) Rydb.

\section{Poaceae}

*Achnatherum constrictum (Hitchc.) Valdés-Reyna \& Barkworth Achnatherum eminens (Cav.) Barkworth

*Agrostis bourgaei E. Fourn.

**Agrostis calderoniae Acosta

Agrostis ghiesbreghtii E. Fourn.

Agrostis hyemalis (Walter) Britton, Sterns \& Poggenb.

*Agrostis liebmannii (E. Fourn.) Hitchc.

Agrostis mertensii Trin.

Agrostis perennans (Walter) Tuck.

*Agrostis schaffneri E. Fourn.

Agrostis subpatens Hitchc.

Agrostis tolucensis Kunth

Amelichloa clandestina (Hack.) Arriaga \& Barkworth

Andropogon bicornis L.

Andropogon gerardi Vitman

Andropogon glomeratus (Walter) Britton, Sterns \& Poggenb.

Andropogon liebmannii Hack.

*Andropogon pringlei Scribn. \& Merr.

Andropogon ternarius Michx.

Anthephora hermaphrodita (L.) Kuntze

*Anthoxanthum mexicanum (Rupr. ex E. Fourn.) Mez

Aristida adscensionis L.

Aristida appressa Vasey

Aristida arizonica Vasey
Aristida capillacea Lam.

Aristida divaricata Humb. \& Bonpl. ex Willd.

Aristida gibbosa (Nees) Kunth

Aristida havardii Vasey

*Aristida hintonii Hitchc.

Aristida jorullensis Kunth

Aristida laxa Cav.

Aristida pansa Wooton \& Standl.

Aristida purpurea Nutt.

Aristida schiedeana Trin. \& Rupr.

Aristida ternipes Cav.

Arundinella berteroniana (Schult.) Hitchc. \& Chase

Arundinella deppeana Nees ex Steud.

Arundinella hispida (Humb. \& Bonpl. ex Willd.) Kuntze

*Aulonemia laxa (F. Maek.) McClure

Avenella flexuosa (L.) Drejer

Axonopus arsenei Swallen

Axonopus compressus (Sw.) P. Beauv.

Bothriochloa barbinodis (Lag.) Herter

Bothriochloa hirtifolia (J. Presl) Henrard

Bothriochloa hybrida (Gould) Gould

Bothriochloa laguroides (DC.) Herter

Bouteloua alamosana Vasey

Bouteloua aristidoides (Kunth) Griseb.

Bouteloua barbata Lag.

Bouteloua chondrosioides (Kunth) Benth. ex S. Watson

Bouteloua curtipendula (Michx.) Torr.

Bouteloua dactyloides (Nutt.) Columbus

Bouteloua dimorpha Columbus

Bouteloua disticha (Kunth) Benth.

Bouteloua gracilis (Kunth) Lag. ex Griffiths

Bouteloua hirsuta Lag.

Bouteloua media (E. Fourn.) Gould \& Kapadia

*Bouteloua multifida (Griffiths) Columbus

*Bouteloua nervata Swallen

Bouteloua parryi (E. Fourn.) Griffiths

*Bouteloua polymorpha (E. Fourn.) Columbus

*Bouteloua purpurea Gould \& Kapadia

Bouteloua repens (Kunth) Scribn. \& Merr.

*Bouteloua scorpioides Lag.

Bouteloua simplex Lag.

Bouteloua triaena (Trin. ex Spreng.) Scribn.

Bouteloua uniflora Vasey

Bouteloua williamsii Swallen

Brachypodium mexicanum (Roem. \& Schult.) Link

Bromus anomalus Rupr. ex E. Fourn. 
Apéndice 4: Continuación.

Bromus carinatus Hook. \& Arn.

Bromus dolichocarpus Wagnon

Bromus exaltatus Bernh.

*Calamagrostis eriantha (Kunth) Steud.

*Calamagrostis orizabae (Rupr. ex E. Fourn.) Beal

Calamagrostis tolucensis (Kunth) Trin. ex Steud.

Cenchrus distachyus (E. Fourn.) Morrone

*Cenchrus durus (Beal) Morrone

Cenchrus echinatus L.

*Cenchrus michoacanus H.F. Gut. \& Morrone

Cenchrus myosuroides Kunth

Cenchrus pilosus Kunth

Cenchrus spinifex Cav.

*Chaboissaea ligulata E. Fourn.

Chaetium bromoides (J. Presl) Benth. ex Hemsl.

Chascolytrum subaristatum (Lam.) Desv.

Chloris rufescens Lag.

Chloris submutica Kunth

*Chusquea bilimekii E. Fourn.

Chusquea cortesii L.G. Clark \& Ruiz-Sanchez

Cinna poiformis (Kunth) Scribn. \& Merr.

Deschampsia elongata (Hook.) Munro

*Deschampsia liebmanniana (E. Fourn.) Hitchc.

Dichanthelium commutatum (Schult.) Gould

Dichanthelium dichotomum (L.) Gould

Dichanthelium ovale (Elliott) Gould \& C.A. Clark

Dichanthelium sphaerocarpon (Elliott) Gould

Diectomis fastigiata (Sw.) P. Beauv.

Digitaria argillacea (Hitchc. \& Chase) Fernald

*Digitaria badia (Scribn. \& Merr.) Fernald

Digitaria bicornis (Lam.) Roem. \& Schult.

Digitaria curtigluma Hitchc.

Digitaria filiformis (L.) Koeler

Digitaria horizontalis Willd.

Digitaria insularis (L.) Mez ex Ekman

Digitaria leucites (Trin.) Henrard

*Digitaria michoacanensis Sánchez-Ken

Dinebra panicea (Retz.) P.M. Peterson \& N. Snow

Diplachne fusca (L.) P. Beauv. ex Roem. \& Schult.

Disakisperma dubium (Kunth) P.M. Peterson \& N. Snow

Distichlis spicata (L.) Greene

Echinochloa crus-pavonis (Kunth) Schult.

Echinochloa holciformis (Kunth) Chase

*Echinochloa jaliscana McVaugh

Echinochloa oplismenoides (E. Fourn.) Hitchc.

Elionurus ciliaris Kunth
Elymus elymoides (Raf.) Swezey

*Elymus pringlei Scribn. \& Merr.

Eragrostis guatemalensis Witherspoon

Eragrostis intermedia Hitchc.

Eragrostis japonica (Thunb.) Trin.

Eragrostis lugens Nees

Eragrostis maypurensis (Kunth) Steud.

Eragrostis mexicana (Hornem.) Link

Eragrostis pectinacea (Michx.) Nees

*Eragrostis plumbea Scribn. ex Beal

Eragrostis swallenii Hitchc.

Eriochloa lemmonii Vasey \& Scribn.

Eriochloa nelsonii Scribn. \& J.G. Sm.

Eriochloa punctata (L.) Desv. ex Ham.

Erioneuron avenaceum (Kunth) Tateoka

Festuca amplissima Rupr.

Festuca breviglumis Swallen

Festuca hephaestophila Nees ex Steud.

Festuca livida (Kunth) Willd. ex Spreng.

Festuca lugens (E. Fourn.) Hitchc. ex Hern.-Xol.

*Festuca orizabensis E.B. Alexeev

Festuca rosei Piper

*Festuca rzedowskiana E.B. Alexeev

Festuca tolucensis Kunth

Festuca willdenowiana Schult. \& Schult. f.

**Festuca $\times$ miscella Darbysh.

Glyceria striata (Lam.) Hitchc.

Heteropogon contortus (L.) P. Beauv. ex Roem. \& Schult.

Heteropogon melanocarpus (Elliott) Benth.

Hilaria belangeri (Steud.) Nash

Hilaria cenchroides Kunth

*Hilaria hintonii Sohns

Hopia obtusa (Kunth) Zuloaga \& Morrone

Ixophorus unisetus (J. Presl) Schltdl.

Jarava ichu Ruiz \& Pav.

Kalinia obtusiflora (E. Fourn.) H.L. Bell \& Columbus

Koeleria pyramidata (Lam.) P. Beauv.

Lasiacis divaricata (L.) Hitchc.

Lasiacis nigra Davidse

Lasiacis procerrima (Hack.) Hitchc.

Lasiacis ruscifolia (Kunth) Hitchc.

Lasiacis sorghoidea (Desv. ex Ham.) Hitchc. \& Chase

Leersia hexandra Sw.

Louisiella elephantipes (Nees ex Trin.) Zuloaga

Luziola fluitans (Michx.) Terrell \& H. Rob.

Luziola peruviana Juss. ex J.F. Gmel. 
Apéndice 4: Continuación.

* Metcalfia mexicana (Scribn.) Conert

Microchloa kunthii Desv.

Mnesithea granularis (L.) de Koning \& Sosef

Morronea parviglumis (Hack.) Zuloaga \& Scataglini

*Muhlenbergia alamosae Vasey

Muhlenbergia brevis C.O. Goodd.

*Muhlenbergia breviseta Griseb. ex E. Fourn.

Muhlenbergia calcicola Swallen

Muhlenbergia capillaris (Lam.) Trin.

Muhlenbergia cenchroides (Humb. \& Bonpl. ex Willd.) P.M. Peterson

Muhlenbergia ciliata (Kunth) Trin.

Muhlenbergia depauperata Scribn.

Muhlenbergia distans Swallen

Muhlenbergia distichophylla (J. Presl) Kunth

Muhlenbergia diversiglumis Trin.

Muhlenbergia dubia E. Fourn.

Muhlenbergia emersleyi Vasey

*Muhlenbergia eriophylla Swallen

*Muhlenbergia gigantea (E. Fourn.) Hitchc.

Muhlenbergia glauca (Nees) B.D. Jacks.

*Muhlenbergia hintonii Swallen

Muhlenbergia implicata (Kunth) Trin.

*Muhlenbergia lucida Swallen

Muhlenbergia macroura (Kunth) Hitchc.

Muhlenbergia microsperma (DC.) Kunth

Muhlenbergia minutissima (Steud.) Swallen

Muhlenbergia montana (Nutt.) Hitchc.

Muhlenbergia nigra Hitchc.

Muhlenbergia orophila Swallen

Muhlenbergia pectinata C.O. Goodd.

Muhlenbergia pereilema P.M. Peterson

Muhlenbergia peruviana (P. Beauv.) Steud.

Muhlenbergia phalaroides (Kunth) P.M. Peterson

Muhlenbergia phleoides (Kunth) Columbus

*Muhlenbergia pilosa P.M. Peterson, Wipff \& S.D. Jones

Muhlenbergia plumbea (Trin.) Hitchc.

Muhlenbergia plumiseta Columbus

Muhlenbergia polycaulis Scribn.

*Muhlenbergia pubescens (Kunth) Hitchc.

Muhlenbergia quadridentata (Kunth) Trin.

Muhlenbergia ramulosa (Kunth) Swallen

Muhlenbergia repens (J. Presl) Hitchc.

Muhlenbergia rigida (Kunth) Kunth

Muhlenbergia robusta (E. Fourn.) Hitchc.

* Muhlenbergia schmitzii Hack.

Muhlenbergia spiciformis Trin.
*Muhlenbergia stricta (J. Presl) Kunth

*Muhlenbergia strictior Scribn. ex Beal

*Muhlenbergia subaristata Swallen

Muhlenbergia tenella (Kunth) Trin.

Muhlenbergia tenuifolia (Kunth) Kunth

Muhlenbergia tricholepis (Torr.) Columbus

Muhlenbergia uniseta (Lag.) Columbus

Muhlenbergia utilis (Torr.) Hitchc.

Muhlenbergia vaginata Swallen

Muhlenbergia versicolor Swallen

Muhlenbergia virescens (Kunth) Trin.

*Muhlenbergia virletii (E. Fourn.) Soderstr.

Munroa pulchella (Kunth) L.D. Amarilla

Nassella leucotricha (Trin. \& Rupr.) R.W. Pohl

Nassella linearifolia (E. Fourn.) R.W. Pohl

Nassella mexicana (Hitchc.) R.W. Pohl

Nassella mucronata (Kunth) R.W. Pohl

Nassella tenuissima (Trin.) Barkworth

Oplismenus burmannii (Retz.) P. Beauv.

Oplismenus compositus (L.) P. Beauv.

Oplismenus hirtellus (L.) P. Beauv.

*Otatea acuminata (Munro) C.E. Calderón \& Soderstr.

*Panicum aztecanum Zuloaga \& Morrone

Panicum capillare L.

*Panicum decolorans Kunth

Panicum dichotomiflorum Michx.

Panicum ghiesbreghtii E. Fourn.

Panicum hallii Vasey

Panicum lepidulum Hitchc. \& Chase

Panicum parcum Hitchc. \& Chase

Panicum plenum Hitchc. \& Chase

Panicum trichoides Sw.

*Panicum vaseyanum Scribn. ex Beal

Pappophorum bicolor E. Fourn.

Paspalum affine Steud.

*Paspalum arsenei Chase

Paspalum botterii (E. Fourn.) Chase

Paspalum conjugatum P.J. Bergius

Paspalum conspersum Schrad.

Paspalum convexum Humb. \& Bonpl. ex Flüggé

Paspalum cymbiforme E. Fourn.

Paspalum denticulatum Trin.

Paspalum distichum $\mathrm{L}$.

Paspalum fimbriatum Kunth

*Paspalum hintonii Chase

Paspalum humboldtianum Flüggé 
Apéndice 4: Continuación.

Paspalum intermedium Munro ex Morong \& Britton

*Paspalum luxurians R. Guzmán \& L. Rico

Paspalum minus E. Fourn.

Paspalum multicaule Poir.

Paspalum notatum Alain ex Flüggé

Paspalum paniculatum $\mathrm{L}$.

Paspalum pilosum Lam.

Paspalum plenum Chase

Paspalum plicatulum Michx.

Paspalum prostratum Scribn. \& Merr.

Paspalum pubiflorum Rupr. ex E. Fourn.

Paspalum setaceum Michx.

Paspalum squamulatum E. Fourn.

Paspalum tenellum Willd.

Paspalum tinctum Chase

**Paspalum tolucense R. Guzmán

Paspalum tumidum Kuhlm.

Paspalum virgatum $\mathrm{L}$.

Peyritschia deyeuxioides (Kunth) Finot

*Peyritschia koelerioides (Peyr.) E. Fourn.

Peyritschia pringlei (Scribn.) S.D. Koch

Phragmites australis (Cav.) Trin. ex Steud.

*Piptochaetium brevicalyx (E. Fourn.) Ricker

Piptochaetium fimbriatum (Kunth) Hitchc.

Piptochaetium seleri (Pilg.) Henrard

Piptochaetium virescens (Kunth) Parodi

Poa calycina (J. Presl) Kunth

Poa chamaeclinos Pilg.

Poa orizabensis Hitchc.

*Poa ruprechtii Peyr.

Poa scaberula Hook. f.

Poa seleri Pilg.

*Poa sharpii Swallen

Polypogon elongatus Kunth

Sacciolepis myuros (Lam.) Chase

Schizachyrium brevifolium (Sw.) Nees ex Büse

Schizachyrium cirratum (Hack.) Wooton \& Standl.

Schizachyrium condensatum (Kunth) Nees

*Schizachyrium mexicanum (Hitchc.) A. Camus

Schizachyrium sanguineum (Retz.) Alston

Schizachyrium semitectum (Swallen) Reeder

Schizachyrium tenerum Nees

Setaria geminata (Forssk.) Veldkamp

Setaria grisebachii E. Fourn.

Setaria liebmannii E. Fourn.

Setaria parviflora (Poir.) Kerguélen
Setariopsis auriculata (E. Fourn.) Scribn.

*Setariopsis latiglumis (Vasey) Scribn.

Sorghastrum incompletum (J. Presl) Nash

Sorghastrum nutans (L.) Nash

*Sporobolus atrovirens (Kunth) Kunth

*Sporobolus hintonii W. Hartley

Sporobolus macrospermus Scribn. ex Beal

Sporobolus pyramidatus (Lam.) Hitchc.

*Sporobolus trichodes Hitchc.

*Sporobolus viscidus Sohns

*Steinchisma cupreum (Hitchc. \& Chase) W.V. Br.

Steinchisma hians (Elliott) Nash

Steinchisma laxum (Sw.) Zuloaga

Tetrapogon chlorideus (J. Presl) P.M. Peterson

Trachypogon spicatus (L. f.) Kuntze

*Triniochloa micrantha (Scribn.) Hitchc.

Triniochloa stipoides (Kunth) Hitchc.

Tripogonella spicata (Nees) P.M. Peterson \& Romasch.

*Tripsacum bravum J.R. Gray

Tripsacum dactyloides (L.) L.

Tripsacum lanceolatum Rupr. ex E. Fourn.

Tripsacum pilosum Scribn. \& Merr.

Trisetum irazuense (Kuntze) Hitchc.

*Trisetum mexicanum (Swallen) S.D. Koch

Trisetum spicatum (L.) K. Richt.

Trisetum viride (Kunth) Kunth

*Trisetum virletii E. Fourn.

Tristachya avenacea (J. Presl) Scribn. \& Merr.

*Urochloa discifera (E. Fourn.) Morrone \& Zuloaga

*Urochloa meziana (Hitchc.) Morrone \& Zuloaga

Urochloa mollis (Sw.) Morrone \& Zuloaga

Zea mays $\mathrm{L}$.

*Zea perennis (Hitchc.) Reeves \& Mangelsd.

Zeugites americanus Willd.

*Zeugites capillaris (Hitchc.) Swallen

*Zeugites hackelii Swallen

*Zeugites hintonii W. Hartley

*Zeugites sagittatus W. Hartley

*Zeugites smilacifolius Scribn.

Zuloagaea bulbosa (Kunth) Bess

\section{Pontederiaceae}

Heteranthera limosa (Sw.) Willd.

Heteranthera peduncularis Benth.

Heteranthera reniformis Ruiz \& Pav.

Heteranthera rotundifolia (Kunth) Griseb. 
Apéndice 4: Continuación.

\section{Potamogetonaceae}

Potamogeton foliosus Raf.

Potamogeton illinoensis Morong

Potamogeton nodosus Poir.

Potamogeton pusillus L.

Stuckenia pectinata (L.) Börner

Zannichellia palustris L.

\section{Ruppiaceae}

Ruppia maritima L.

\section{Smilacaceae}

Smilax laurifolia $\mathrm{L}$.

Smilax mollis Humb. \& Bonpl. ex Willd.

Smilax moranensis M. Martens \& Galeotti

*Smilax pringlei Greenm.

Smilax subpubescens A. DC.

\section{Typhaceae}

Sparganium eurycarpum Engelm.

Typha domingensis Pers.

Typha latifolia L.

\section{EUDICOTILEDÓNEAS}

\section{Acanthaceae}

Anisacanthus quadrifidus (Vahl) Nees

Aphelandra aurantiaca (Scheidw.) Lindl.

* Aphelandra lineariloba Leonard

Barleria oenotheroides Dum. Cours.

* Carlowrightia pectinata Brandegee

* Dicliptera inaequalis Greenm.

* Dicliptera peduncularis Nees

Dicliptera resupinata (Vahl) A. Juss.

* Dicliptera thlaspioides Nees

* Dyschoriste hirsutissima (Nees) Kuntze

* Dyschoriste microphylla (Cav.) Kuntze

Dyschoriste ovata (Cav.) Kuntze

Dyschoriste quadrangularis (Oerst.) Kuntze

Dyschoriste schiedeana (Nees) Kuntze

Elytraria imbricata (Vahl) Pers.

* Elytraria mexicana Fryxell \& S.D. Koch

Henrya insularis Nees

Justicia aurea Schltdl.

Justicia caudata A. Gray

Justicia fulvicoma Schltdl. \& Cham.

*Justicia furcata Jacq.
Justicia pringlei B.L. Rob.

*Justicia salviiflora Kunth

Justicia spicigera Schltdl.

*Poikilacanthus capitatus (Leonard) Ramamoorthy

Pseuderanthemum cuspidatum (Nees) Radlk.

Pseuderanthemum praecox (Benth.) Leonard

Pseuderanthemum standleyi Leonard

Ruellia blechum L.

*Ruellia bourgaei Hemsl.

Ruellia hookeriana (Nees) Hemsl.

Ruellia inundata Kunth

Ruellia lactea Cav.

Ruellia parva (Nees) Hemsl.

* Ruellia speciosa (Nees) Lindau

Ruellia spissa Leonard

Stenandrium dulce (Cav.) Nees

*Stenandrium verticillatum Brandegee

* Tetramerium glandulosum Oerst.

Tetramerium nervosum Nees

Tetramerium tenuissimum Rose

\section{Actinidiaceae}

*Saurauia serrata DC.

Saurauia yasicae Loes.

\section{Adoxaceae}

Sambucus nigra L.

Viburnum acutifolium Benth.

*Viburnum ciliatum Greenm.

*Viburnum elatum Benth.

*Viburnum loeseneri Graebn.

*Viburnum stenocalyx (Oerst.) Hemsl.

\section{Aizoaceae}

Sesuvium portulacastrum (L.) L.

Trianthema portulacastrum L.

\section{Altingiaceae}

Liquidambar styraciflua L.

\author{
Amaranthaceae \\ Alternanthera caracasana Kunth \\ Alternanthera pungens Kunth \\ Amaranthus acutilobus Uline \& W.L. Bray \\ Amaranthus hybridus L. \\ Amaranthus hypochondriacus L.
}


Apéndice 4: Continuación.

Amaranthus palmeri S. Watson

Amaranthus powellii S. Watson

Amaranthus spinosus L.

*Atriplex linifolia Humb. \& Bonpl. ex Willd.

*Atriplex muricata Humb. \& Bonpl. ex Willd.

Chamissoa altissima (Jacq.) Kunth

Chenopodium berlandieri Moq.

Chenopodium fremontii $\mathrm{S}$. Watson

*Chenopodium mexicanum Moq.

*Chenopodium nuttalliae Saff.

Chenopodium pratericola Rydb.

Dysphania ambrosioides (L.) Mosyakin \& Clemants

Dysphania graveolens (Willd.) Mosyakin \& Clemants

Gomphrena nitida Rothr.

*Gomphrena parviceps Standl.

* Gomphrena pringlei J.M. Coult. \& Fisher

Gomphrena serrata L.

Guilleminea densa (Humb. \& Bonpl. ex Schult.) Moq.

* Iresine ajuscana Suess. \& Beyerle

Iresine arbuscula Uline \& W.L. Bray

Iresine calea (Ibáñez) Standl.

*Iresine cassiniiformis S. Schauer

Iresine diffusa Humb. \& Bonpl. ex Willd.

Iresine grandis Standl.

Iresine herbstii Hook.

Iresine heterophylla Standl.

Iresine interrupta Benth.

Suaeda mexicana (Standl.) Standl.

Suaeda nigra (Raf.) J.F. Macbr.

\section{Anacardiaceae}

* Actinocheita filicina (DC.) F.A. Barkley

*Amphipterygium adstringens (Schltdl.) Standl.

* Comocladia mollissima Kunth

* Cyrtocarpa procera Kunth

Pistacia mexicana Kunth

*Pseudosmodingium andrieuxii (Baill.) Engl.

* Pseudosmodingium perniciosum (Kunth) Engl.

* Pseudosmodingium virletii (Baill.) Engl.

Rhus aromatica Aiton

Rhus schiedeana Schltdl.

*Rhus standleyi F.A. Barkley

Rhus terebinthifolia Schltdl. \& Cham.

Rhus virens Lindh. ex A. Gray

Spondias purpurea $\mathrm{L}$.

Toxicodendron radicans (L.) Kuntze

\author{
Apiaceae \\ *Angelica nelsonii J.M. Coult. \& Rose \\ Arracacia aegopodioides (Kunth) J.M. Coult. \& Rose \\ Arracacia atropurpurea (Lehm.) Benth. \& Hook. f. ex Hemsl. \\ *Arracacia longipedunculata J.M. Coult. \& Rose \\ *Arracacia quadrifida Constance \& Affolter \\ * Arracacia rigida J.M. Coult. \& Rose \\ Arracacia tolucensis (Kunth) Hemsl. \\ Bowlesia flabilis J.F. Macbr. \\ *Chaerophyllum orizabae (I.M. Johnst.) K.F. Chung \\ *Chaerophyllum tolucanum (I.M. Johnst.) K.F. Chung \\ Cyclospermum leptophyllum (Pers.) Sprague ex Britton \& P. Wilson \\ Daucus montanus Humb. \& Bonpl. ex Spreng. \\ *Donnellsmithia biennis (J.M. Coult. \& Rose) Mathias \& Constance \\ * Donnellsmithia hintonii Mathias \& Constance \\ Donnellsmithia juncea (Spreng.) Mathias \& Constance \\ * Donnellsmithia mexicana (B.L. Rob.) Mathias \& Constance \\ * Donnellsmithia serrata (J.M. Coult. \& Rose) Mathias \& Constance \\ Enantiophylla heydeana J.M. Coult. \& Rose \\ *Eryngium alternatum J.M. Coult. \& Rose \\ *Eryngium beecheyanum Hook. \& Arn. \\ *Eryngium bonplandii F. Delaroche \\ Eryngium carlinae F. Delaroche \\ *Eryngium cervantesii F. Delaroche \\ *Eryngium columnare Hemsl. \\ *Eryngium comosum F. Delaroche \\ Eryngium cymosum F. Delaroche \\ *Eryngium deppeanum Schltdl. \& Cham. \\ *Eryngium galeottii Hemsl. \\ Eryngium ghiesbreghtii Decne. \\ Eryngium gracile F. Delaroche \\ Eryngium heterophyllum Engelm. \\ Eryngium humile Cav. \\ *Eryngium longifolium Cav. \\ *Eryngium monocephalum Cav. \\ *Eryngium pectinatum C. Presl ex DC. \\ Eryngium phyteumae F. Delaroche \\ *Eryngium proteiflorum F. Delaroche \\ *Eryngium serratum Cav. \\ *Eryngium subacaule Cav. \\ Lilaeopsis schaffneriana (Schltdl.) J.M. Coult. \& Rose \\ Micropleura renifolia Lag. \\ *Neogoezia gracilipes (Hemsl.) Hemsl. \\ *Neogoezia planipetala (Hemsl.) Hemsl. \\ Osmorhiza mexicana Griseb. \\ *Prionosciadium acuminatum B.L. Rob.
}


Apéndice 4: Continuación.

*Prionosciadium cuneatum J.M. Coult. \& Rose

*Prionosciadium diversifolium Rose

* Prionosciadium nelsonii J.M. Coult. \& Rose

Prionosciadium thapsoides (DC.) Mathias

Rhodosciadium diffusum (J.M. Coult. \& Rose) Mathias \& Constance

Rhodosciadium glaucum J.M. Coult. \& Rose

* Rhodosciadium purpureum (Rose) Mathias \& Constance

* Rhodosciadium tolucense (Kunth) Mathias

*Rhodosciadium tuberosum (J.M. Coult. \& Rose) Drude

Sanicula liberta Cham. \& Schltdl.

Spananthe paniculata Jacq.

*Tauschia alpina (J.M. Coult. \& Rose) Mathias

*Tauschia decumbens (Benth.) J.M. Coult. \& Rose

*Tauschia humilis J.M. Coult. \& Rose

**Tauschia neglecta Calderón \& Constance

Tauschia nudicaulis Schltdl.

\section{Apocynaceae}

Allamanda cathartica $\mathrm{L}$.

Asclepias angustifolia Schweigg.

Asclepias auriculata Kunth

Asclepias curassavica L.

*Asclepias fournieri Woodson

Asclepias glaucescens Kunth

Asclepias jaliscana B.L. Rob.

Asclepias linaria Cav.

*Asclepias lynchiana Fishbein

*Asclepias mexicana Cav.

*Asclepias notha W.D. Stevens

*Asclepias nummularioides W.D. Stevens

Asclepias oenotheroides Schltdl. \& Cham.

*Asclepias otarioides E. Fourn.

*Asclepias ovata M. Martens \& Galeotti

Asclepias pellucida E. Fourn.

*Asclepias pringlei (Greenm.) Woodson

Asclepias quinquedentata A. Gray

Asclepias similis Hemsl.

Blepharodon mucronatum (Schltdl.) Decne.

Cascabela ovata (Cav.) Lippold

Cascabela thevetia (L.) Lippold

* Cascabela thevetioides (Kunth) Lippold

* Cynanchum foetidum (Cav.) Kunth

Cynanchum ligulatum (Benth.) Woodson

Dictyanthus asper (Mill.) W.D. Stevens

Dictyanthus parviflorus Hemsl.

*Dictyanthus pavonii Decne.
*Dictyanthus reticulatus (Turcz.) Benth. \& Hook. f. ex Hemsl.

*Dictyanthus tigrinus Conz. \& Standl.

*Fernaldia asperoglottis Woodson

Fernaldia pandurata (A. DC.) Woodson

Funastrum bilobum (Hook. \& Arn.) J.F. Macbr.

Funastrum clausum (Jacq.) Schltr.

*Funastrum elegans (Decne.) Schltr.

*Funastrum pannosum (Decne.) Schltr.

Gonolobus barbatus Kunth

*Gonolobus chloranthus Schltdl.

Gonolobus erianthus Decne.

Gonolobus fraternus Schltdl.

*Gonolobus grandiflorus (Cav.) R. Br. ex Roem. \& Schult.

*Gonolobus megalocarpus Paul G. Wilson

*Gonolobus pectinatus Brandegee

* Gonolobus sororius A. Gray

*Gonolobus spiranthus Juárez-Jaimes, W.D. Stevens \& Lozada-Pérez

Gonolobus uniflorus Kunth

Haplophyton cimicidum A. DC.

* Laubertia contorta (M. Martens \& Galeotti) Woodson

*Mandevilla foliosa (Müll. Arg.) Hemsl.

* Mandevilla holosericea (Sessé \& Moc.) J.K. Williams

Mandevilla hypoleuca (Benth.) Pichon

* Mandevilla mexicana (Müll. Arg.) Woodson

Mandevilla subsagittata (Ruiz \& Pav.) Woodson

Mandevilla torosa (Jacq.) Woodson

Mandevilla tubiflora (M. Martens \& Galeotti) Woodson

Marsdenia bourgaeana (Baill.) W. Rothe

* Marsdenia edulis S. Watson

Marsdenia lanata (Paul G. Wilson) W.D. Stevens

Marsdenia mexicana Decne.

Marsdenia trivirgulata Bartlett

* Marsdenia tubularis L.O. Williams

*Marsdenia zimapanica Hemsl.

* Matelea chrysantha (Greenm.) Woodson

* Matelea congesta (Decne.) Woodson

* Matelea crenata (Vail) Woodson

* Matelea decumbens W.D. Stevens

Matelea gonoloboides (B.L. Rob. \& Greenm.) Woodson

* Matelea nummularia (Decne.) Woodson

* Matelea pedunculata (Decne.) Woodson

*Matelea pilosa (Benth.) Woodson

*Metastelma lanceolatum Schltr.

Metastelma schlechtendalii Decne.

* Orthosia angustifolia (Turcz.) Liede \& Meve

*Orthosia pubescens (Greenm.) Liede \& Meve 
Apéndice 4: Continuación.

Pentalinon andrieuxii (Müll. Arg.) B.F. Hansen \& Wunderlin

Pherotrichis villosa Meisn.

Plumeria rubra L.

Polystemma guatemalense (Schltr.) W.D. Stevens

Prestonia mexicana A. DC.

Rauvolfia tetraphylla $\mathrm{L}$.

* Suberogerens cyclophylla (Standl.) Morillo

Tabernaemontana alba Mill.

Tabernaemontana donnell-smithii Rose

Tabernaemontana odontadeniiflora A.O. Simões \& M.E. Endress

Tabernaemontana tomentosa (Greenm.) A.O. Simões \& M.E. Endress

* Thenardia floribunda Kunth

*Thenardia galeottiana Baill.

Tonduzia longifolia (A. DC.) Markgr.

\section{Apodanthaceae}

Pilostyles thurberi A. Gray

\section{Aquifoliaceae}

Ilex brandegeana Loes.

Ilex discolor Hemsl.

*Ilex mexicana (Turcz.) Black ex Hemsl.

\section{Araliaceae}

Aralia humilis Cav.

Dendropanax arboreus (L.) Decne. \& Planch.

Hydrocotyle mexicana Schltdl. \& Cham.

Hydrocotyle ranunculoides $\mathrm{L}$. $\mathrm{f}$.

Hydrocotyle umbellata $\mathrm{L}$.

Hydrocotyle verticillata Thunb.

Oreopanax echinops (Cham. \& Schltdl.) Decne. \& Planch.

Oreopanax peltatus Linden ex Regel

Oreopanax xalapensis (Kunth) Decne. \& Planch.

\section{Asteraceae}

Achillea millefolium L.

* Achyropappus anthemoides Kunth

Acmella radicans (Jacq.) R.K. Jansen

Acmella repens (Walter) Rich.

* Acourtia cordata (Cerv.) B.L. Turner

*Acourtia cuernavacana (B.L. Rob. \& Greenm.) Reveal \& R.M.

King

*Acourtia dugesii (A. Gray) Reveal \& R.M. King

*Acourtia fruticosa (Lex.) B.L. Turner

*Acourtia glomeriflora (A. Gray) Reveal \& R.M. King

*Acourtia humboldtii (Less.) B.L. Turner
*Acourtia lozanoi (Greenm.) Reveal \& R.M. King

*Acourtia moschata (La Llave \& Lex) DC.

*Acourtia platyptera (B.L. Rob.) Reveal \& R.M. King

Acourtia reticulata (Lag. ex D. Don) Reveal \& R.M. King

*Acourtia turbinata (Lex.) DC.

*Acourtia wislizeni (A. Gray) Reveal \& R.M. King

*Adenophyllum glandulosum (Cav.) Strother

Adenophyllum porophyllum (Cav.) Hemsl.

*Adenophyllum pulcherrimum (Strother) Villarreal

Ageratina adenophora (Spreng.) R.M. King \& H. Rob.

Ageratina areolaris (DC.) Gage ex B.L. Turner

Ageratina bellidifolia (Benth.) R.M. King \& H. Rob.

*Ageratina brevipes (DC.) R.M. King \& H. Rob.

*Ageratina calophylla (Greene) R.M. King \& H. Rob.

*Ageratina cardiophylla (B.L. Rob.) R.M. King \& H. Rob.

Ageratina chiapensis (B.L. Rob.) R.M. King \& H. Rob.

* Ageratina choricephala (B.L. Rob.) R.M. King \& H. Rob.

Ageratina conspicua (Kunth \& C.D. Bouché) R.M. King \& H. Rob.

Ageratina crassiramea (B.L. Rob.) R.M. King \& H. Rob.

*Ageratina cylindrica (McVaugh) R.M. King \& H. Rob.

*Ageratina deltoidea (Jacq.) R.M. King \& H. Rob.

*Ageratina enixa (B.L. Rob.) R.M. King \& H. Rob.

*Ageratina espinosarum (A. Gray) R.M. King \& H. Rob.

* Ageratina glabrata (Kunth) R.M. King \& H. Rob.

*Ageratina irrasa (B.L. Rob.) R.M. King \& H. Rob.

*Ageratina isolepis (B.L. Rob.) R.M. King \& H. Rob.

*Ageratina leiocarpa (B.L. Rob.) Gage ex B.L. Turner

*Ageratina liebmannii (Sch. Bip. ex Klatt) R.M. King \& H. Rob.

Ageratina ligustrina (DC.) R.M. King \& H. Rob.

*Ageratina lucida (Ortega) R.M. King \& H. Rob.

*Ageratina macvaughii R.M. King \& H. Rob.

Ageratina mairetiana (DC.) R.M. King \& H. Rob.

*Ageratina malacolepis (B.L. Rob.) R.M. King \& H. Rob.

*Ageratina moorei B.L. Turner

Ageratina muelleri (Sch. Bip. ex Klatt) R.M. King \& H. Rob.

*Ageratina neohintoniorum B.L. Turner

*Ageratina oligocephala (DC.) R.M. King \& H. Rob.

*Ageratina oreithales (Greenm.) B.L. Turner

*Ageratina parayana (J. Espinosa) B.L. Turner

Ageratina pazcuarensis (Kunth) R.M. King \& H. Rob.

*Ageratina petiolaris (Moc. \& Sessé ex DC.) R.M. King \& H. Rob. Ageratina pichinchensis (Kunth) R.M. King \& H. Rob.

Ageratina prunellifolia (Kunth) R.M. King \& H. Rob.

* Ageratina ramireziorum (J. Espinosa) B.L. Turner

*Ageratina rhomboidea (Kunth) R.M. King \& H. Rob.

*Ageratina rubricaulis (Kunth) R.M. King \& H. Rob. 
Apéndice 4: Continuación.

*Ageratina scorodonioides (A. Gray) R.M. King \& H. Rob. Ageratina tomentella (Schrad.) R.M. King \& H. Rob. Ageratina vernalis (Vatke \& Kurtz) R.M. King \& H. Rob. * Ageratina vernicosa (Sch. Bip. ex Greenm.) R.M. King \& H. Rob. Ageratina wrightii (A. Gray) R.M. King \& H. Rob. Ageratum corymbosum Zuccagni

* Aldama buddleiiformis (DC.) E.E. Schill. \& Panero Aldama dentata La Llave

* Aldama excelsa (Willd.) E.E. Schill. \& Panero

* Aldama flava (Hemsl.) E.E. Schill. \& Panero

*Aldama ghiesbreghtii (Hemsl.) E.E. Schill. \& Panero

* Aldama hispida (Kunth) E.E. Schill. \& Panero

* Aldama hypochlora (S.F. Blake) E.E. Schill. \& Panero

*Aldama linearis (Cav.) E.E. Schill. \& Panero

*Aldama morelensis (Greenm.) E.E. Schill. \& Panero

Alloispermum integrifolium (DC.) H. Rob.

*Alloispermum michoacanum (B.L. Rob.) B.L. Turner

Alloispermum scabrum (Lag.) H. Rob.

Almutaster pauciflorus (Nutt.) Á. Löve \& D. Löve

* Alomia ageratoides Kunth

*Alomia alata Hemsl.

* Ambrosia canescens A. Gray

Ambrosia confertiflora DC.

Ambrosia peruviana Willd.

Aphanostephus ramosissimus DC.

Archibaccharis asperifolia (Benth.) S.F. Blake

*Archibaccharis auriculata (Hemsl.) G.L. Nesom

*Archibaccharis hieracioides (S.F. Blake) S.F. Blake

Archibaccharis hirtella (DC.) Heering

Archibaccharis schiedeana (Benth.) J.D. Jacks.

Archibaccharis serratifolia (Kunth) S.F. Blake

*Artemisia klotzschiana Besser

Artemisia ludoviciana Nutt.

*Asanthus thyrsiflorus (A. Gray) R.M. King \& H. Rob.

*Astranthium orthopodum (B.L. Rob. \& Fernald) Larsen

**Astranthium reichei $\mathrm{Rzed}$.

Baccharis conferta Kunth

*Baccharis erosoricola Rzed.

Baccharis heterophylla Kunth

*Baccharis macrocephala Sch. Bip. ex Greenm.

Baccharis multiflora Kunth

Baccharis pteronioides DC.

Baccharis salicifolia (Ruiz \& Pav.) Pers.

*Baccharis sordescens DC.

Baccharis thesioides Kunth

Baccharis trinervis (Lam.) Pers.
*Bahia pringlei Greenm.

Baltimora geminata (Brandegee) Stuessy

Barkleyanthus salicifolius (Kunth) H. Rob. \& Brettell

Bartlettina oresbia (B.L. Rob.) R.M. King \& H. Rob.

Bartlettina sordida (Less.) R.M. King \& H. Rob.

*Bidens aequisquama (Fernald) Sherff

Bidens alba (L.) DC.

*Bidens angustissima Kunth

*Bidens anthemoides (DC.) Sherff

Bidens aurea (Aiton) Sherff

Bidens bigelovii A. Gray

Bidens laevis (L.) Britton, Sterns \& Poggenb.

Bidens lemmonii A. Gray

Bidens odorata Cav.

Bidens ostruthioides (DC.) Sch. Bip.

Bidens pilosa L.

Bidens rostrata Melchert

*Bidens schaffneri (A. Gray) Sherff

*Bidens serrulata (Poir.) Desf.

Bidens triplinervia Kunth

Blumea viscosa (Mill.) V.M. Badillo

* Boeberoides grandiflora (DC.) Strother

*Brickellia cavanillesii (Cass.) A. Gray

Brickellia diffusa (Vahl) A. Gray

Brickellia eupatorioides (L.) Shinners

Brickellia glandulosa (La Llave) McVaugh

*Brickellia glomerata Fernald

*Brickellia monocephala B.L. Rob.

*Brickellia nutanticeps S.F. Blake

Brickellia oliganthes (Less.) A. Gray

*Brickellia pavonii (A. Gray) B.L. Turner

*Brickellia pedunculosa (DC.) Harc. \& Beaman

*Brickellia pendula (Schrad.) A. Gray

Brickellia scoparia (DC.) A. Gray

*Brickellia secundiflora (Lag.) A. Gray

* Brickellia squarrosa B.L. Rob. \& Seaton

*Brickellia subuligera (S. Schauer) B.L. Turner

*Brickellia tomentella A. Gray

Brickellia veronicifolia (Kunth) A. Gray

Calea ternifolia Kunth

Calea urticifolia (Mill.) DC.

Calyptocarpus vialis Less.

Calyptocarpus wendlandii Sch. Bip.

*Carminatia alvarezii Rzed. \& Calderón

Carminatia recondita McVaugh

Carminatia tenuiflora DC. 
Apéndice 4: Continuación.

* Carphochaete grahamii A. Gray

Centaurea rothrockii Greenm.

Chaetopappa ericoides (Torr.) G.L. Nesom

* Chaptalia hintonii Bullock

Chaptalia nutans (L.) Pol.

Chaptalia piloselloides (Vahl) Baker

Chaptalia transiliens G.L. Nesom

*Chionolaena lavandulifolia (Kunth) Benth. \& Hook. f. ex B.D. Jacks.

Chionolaena salicifolia (Bertol.) G.L. Nesom

Chloracantha spinosa (Benth.) G.L. Nesom

Chromolaena collina (DC.) R.M. King \& H. Rob.

Chromolaena odorata (L.) R.M. King \& H. Rob.

* Chromolaena pulchella (Kunth) R.M. King \& H. Rob.

* Chromolepis heterophylla Benth.

Chrysactinia mexicana A. Gray

Chrysanthellum indicum DC.

*Chrysanthellum involutum Paul G. Wilson

*Cirsium acantholepis (Hemsl.) Petr.

* Cirsium anartiolepis Petr.

*Cirsium conspicuum (G. Don) Sch. Bip.

*Cirsium ehrenbergii Sch. Bip.

*Cirsium jorullense (Kunth) Spreng.

*Cirsium lomatolepis (Hemsl.) Petr.

Cirsium mexicanum DC.

*Cirsium nivale (Kunth) Sch. Bip.

*Cirsium pascuarense (Kunth) Spreng.

* Cirsium pinetorum Greenm.

*Cirsium rhaphilepis (Hemsl.) Petr.

Cirsium subcoriaceum (Less.) Sch. Bip.

*Cirsium subuliforme G.B. Ownbey

*Cirsium tolucanum (B.L. Rob. \& Seaton) Petr.

*Cirsium velatum (S. Watson) Petr.

Conyza bonariensis (L.) Cronquist

Conyza canadensis (L.) Cronquist

Conyza coronopifolia Kunth

*Conyza microcephala Hemsl.

*Coreopsis petrophiloides B.L. Rob. \& Greenm.

*Coreopsis rhyacophila Greenm.

Cosmos bipinnatus Cav.

Cosmos caudatus Kunth

Cosmos crithmifolius Kunth

Cosmos diversifolius Otto

* Cosmos modestus Sherff

**Cosmos nitidus Paray

*Cosmos pacificus Melchert
Cosmos parviflorus (Jacq.) Pers.

*Cosmos purpureus (DC.) Benth. \& Hook. f. ex Hemsl.

*Cosmos scabiosoides Kunth

* Cosmos schaffneri Sherff

Cosmos sulphureus Cav.

Cotula mexicana (DC.) Cabrera

Critonia hebebotrya DC.

Critonia quadrangularis (DC.) R.M. King \& H. Rob.

*Critoniopsis pallens (Sch. Bip.) H. Rob.

*Critoniopsis salicifolia (DC.) H. Rob.

*Critoniopsis tomentosa (Lex.) H. Rob.

*Critoniopsis uniflora (Sch. Bip.) H. Rob.

*Dahlia atropurpurea P.D. Sørensen

*Dahlia brevis P.D. Sørensen

Dahlia coccinea Cav.

Dahlia excelsa Benth.

*Dahlia merckii Lehm.

* Dahlia neglecta Saar

Dahlia pinnata Cav.

* Dahlia rudis P.D. Sørensen

*Dahlia scapigera (A. Dietr.) Knowles \& Westc.

Dahlia sorensenii H.V. Hansen \& Hjert.

Decachaeta incompta (DC.) R.M. King \& H. Rob.

*Decachaeta ovatifolia (DC.) R.M. King \& H. Rob.

* Decachaeta perornata (Klatt) R.M. King \& H. Rob.

*Decachaeta pyramidalis (B.L. Rob.) S.D. Sundb., C.P. Cowan \&

B.L. Turner

Delilia biflora (L.) Kuntze

*Dendroviguiera pringlei (Fernald) E.E. Schill. \& Panero

*Dendroviguiera sphaerocephala (DC.) E.E. Schill. \& Panero

*Desmanthodium fruticosum Greenm.

*Desmanthodium ovatum Benth.

*Dugesia mexicana (A. Gray) A. Gray

Dyssodia papposa (Vent.) Hitchc.

* Dyssodia pinnata (Cav.) B.L. Rob.

* Dyssodia tagetiflora Lag.

Eclipta prostrata (L.) L.

Electranthera mutica (DC.) Mesfin, D.J. Crawford \& Pruski

Elephantopus mollis Kunth

Erechtites hieraciifolius (L.) Raf. ex DC.

Erigeron delphinifolius Willd.

*Erigeron galeottii (A. Gray ex Hemsl.) Greene

*Erigeron janivultus G.L. Nesom

Erigeron karvinskianus DC.

Erigeron longipes DC.

*Erigeron polycephalus (Larsen) G.L. Nesom 
Apéndice 4: Continuación.

Erigeron pubescens Kunth

Erigeron versicolor (Greenm.) G.L. Nesom

*Eupatoriastrum triangulare (DC.) B.L. Rob.

*Euphrosyne partheniifolia DC.

*Flaveria angustifolia (Cav.) Pers.

Flaveria trinervia (Spreng.) C. Mohr

Fleischmannia arguta (Kunth) B.L. Rob.

* Fleischmannia holwayana (B.L. Rob.) R.M. King \& H. Rob. Fleischmannia pycnocephala (Less.) R.M. King \& H. Rob.

*Florestina lobata B.L. Turner

Florestina pedata (Cav.) Cass.

Galeana pratensis (Kunth) Rydb.

* Galinsoga longipes Canne

Galinsoga parviflora Cav.

Gamochaeta americana (Mill.) Wedd.

Gamochaeta falcata (Lam.) Cabrera

Gamochaeta purpurea (L.) Cabrera

Gamochaeta sphacelata (Kunth) Cabrera

Gochnatia hypoleuca (DC.) A. Gray

* Grindelia inuloides Willd.

*Grindelia tricuspis (Sch. Bip.) Adr. Bartoli \& Tortosa

* Guardiola mexicana Bonpl.

* Gutierrezia alamanii A. Gray

Gymnosperma glutinosum (Spreng.) Less.

Helenium mexicanum Kunth

Helenium quadridentatum Labill.

Helenium scorzonerifolium (DC.) A. Gray

Helianthus annuus L.

Helianthus laciniatus A. Gray

*Heliopsis annua Hemsl.

Heliopsis buphthalmoides (Jacq.) Dunal

*Heliopsis procumbens Hemsl.

Heterosperma pinnatum Cav.

*Heterotheca inuloides Cass.

Hieracium abscissum Less.

Hieracium crepidispermum Fr.

*Hieracium dysonymum S.F. Blake

Hieracium fendleri Sch. Bip.

*Hieracium hintonii Beaman

Hieracium mexicanum Less.

Hieracium pringlei A. Gray

Hieracium schultzii Fr.

*Hofmeisteria dissecta (Hook. \& Arn.) R.M. King \& H. Rob.

*Hofmeisteria schaffneri (A. Gray) R.M. King \& H. Rob.

*Hybridella globosa (Ortega) Cass.

Hymenostephium cordatum (Hook. \& Arn.) S.F. Blake
Hymenostephium tenuis (A. Gray) E.E. Schill. \& Panero

*Hymenostephium uniseriatum E.E. Schill. \& Panero

*Hymenoxys chrysanthemoides (Kunth) DC.

Hymenoxys integrifolia (Kunth) Bierner

*Iostephane heterophylla (Cav.) Hemsl.

*Iostephane trilobata Hemsl.

*Isocoma veneta (Kunth) Greene

*Jaegeria bellidiflora (Moc. \& Sessé ex DC.) Torres \& Beaman

*Jaegeria glabra (S. Watson) B.L. Rob.

Jaegeria hirta (Lag.) Less.

*Jaegeria macrocephala Less.

*Jaegeria pedunculata Hook. \& Arn.

*Koanophyllon monanthum (Sch. Bip.) T.J. Ayers \& B.L. Turner

*Lactuca brachyrrhyncha Greenm.

Laennecia confusa (Cronquist) G.L. Nesom

Laennecia filaginoides DC.

Laennecia gnaphalioides (Kunth) Cass.

Laennecia schiedeana (Less.) G.L. Nesom

Laennecia sophiifolia (Kunth) G.L. Nesom

Lagascea helianthifolia Kunth

*Lagascea heteropappus Hemsl.

* Lagascea rigida (Cav.) Stuessy

*Lasianthaea aurea (D. Don) K.M. Becker

*Lasianthaea ceanothifolia (Willd.) K.M. Becker

*Lasianthaea crocea (A. Gray) K.M. Becker

Lasianthaea fruticosa (L.) K.M. Becker

*Lasianthaea helianthoides DC.

Leibnitzia lyrata (D. Don) G.L. Nesom

Melampodium americanum L.

* Melampodium aureum Brandegee

Melampodium bibracteatum S. Watson

Melampodium diffusum Cass.

Melampodium divaricatum (Rich.) DC.

*Melampodium glabrum S. Watson

Melampodium gracile Less.

Melampodium linearilobum DC.

*Melampodium longifolium Cerv. ex Cav.

Melampodium microcephalum Less.

Melampodium montanum Benth.

Melampodium perfoliatum (Cav.) Kunth

*Melampodium repens Sessé \& Moc.

Melampodium sericeum Lag.

Melampodium strigosum Stuessy

*Melampodium tenellum Hook. \& Arn.

* Mexerion sarmentosum (Klatt) G.L. Nesom

*Microspermum debile Benth. 
Apéndice 4: Continuación.

* Microspermum flaccidum Paul G. Wilson

* Microspermum nummulariifolium Lag.

Mikania cordifolia (L. f.) Willd.

Milleria quinqueflora $\mathrm{L}$.

*Montanoa bipinnatifida (Kunth) K. Koch

*Montanoa frutescens Mairet ex DC.

*Montanoa grandiflora Alamán ex DC.

*Montanoa karwinskii DC.

Montanoa leucantha (Lag.) S.F. Blake

Montanoa tomentosa Cerv.

Osbertia stolonifera (DC.) Greene

* Oteiza acuminata La Llave

*Otopappus epaleaceus Hemsl.

*Otopappus imbricatus (Sch. Bip.) S.F. Blake

*Otopappus tequilanus (A. Gray) B.L. Rob.

Oxylobus adscendens (Sch. Bip. ex Hemsl.) B.L. Rob. \& Greenm.

Oxylobus arbutifolius (Kunth) A. Gray

* Packera bellidifolia (Kunth) W.A. Weber \& Á. Löve

* Packera sanguisorbae (DC.) C. Jeffrey

* Packera toluccana (DC.) W.A. Weber \& Á. Löve

Parthenium bipinnatifidum (Ortega) Rollins

Parthenium hysterophorus L.

Parthenium incanum Kunth

*Pectis haenkeana (DC.) Sch. Bip.

Pectis prostrata Cav.

*Pectis schaffneri Sch. Bip. ex Fernald

Pectis uniaristata DC.

*Perymenium berlandieri DC.

*Perymenium buphthalmoides DC.

*Perymenium globosum B.L. Rob.

*Perymenium macrocephalum Greenm.

*Perymenium mendezii DC.

*Perymenium reticulatum J.J. Fay

*Perymenium rogmacvaughii Rzed. \& Calderón

Pinaropappus roseus (Less.) Less.

* Piqueria pilosa Kunth

* Piqueria triflora Hemsl.

Piqueria trinervia Cav.

*Pittocaulon bombycophole (Bullock) H. Rob. \& Brettell

* Pittocaulon praecox (Cav.) H. Rob. \& Brettell

Pittocaulon velatum (Greenm.) H. Rob. \& Brettell

Pluchea salicifolia (Mill.) S.F. Blake

*Porophyllum calcicola B.L. Rob. \& Greenm.

* Porophyllum linaria (Cav.) DC.

Porophyllum macrocephalum DC.

*Porophyllum pringlei B.L. Rob.
*Porophyllum viridiflorum (Kunth) DC.

*Porophyllum warnockii R.R. Johnson

*Psacalium amplifolium (DC.) H. Rob. \& Brettell

*Psacalium cirsiifolium (Zucc.) H. Rob. \& Brettell

*Psacalium goldsmithii (B.L. Rob.) H. Rob. \& Brettell

**Psacalium hintonii (Pippen) H. Rob. \& Brettell

*Psacalium holwayanum (B.L. Rob.) Rydb.

* Psacalium laxiflorum Benth.

*Psacalium matudae H. Rob. \& Brettell

*Psacalium megaphyllum (B.L. Rob. \& Greenm.) Rydb.

*Psacalium peltatum (Kunth) Cass.

* Psacalium silphiifolium (B.L. Rob. \& Greenm.) H. Rob. \& Brettell

*Psacalium sinuatum (Cerv.) H. Rob. \& Brettell

* Psacalium tussilaginoides (Kunth) H. Rob. \& Brettell

Pseudelephantopus spicatus (Juss. ex Aubl.) C.F. Baker

*Pseudognaphalium altamiranum (Greenm.) Anderb.

Pseudognaphalium attenuatum (DC.) Anderb.

*Pseudognaphalium bourgovii (A. Gray) Anderb.

Pseudognaphalium brachypterum (DC.) Anderb.

Pseudognaphalium canescens (DC.) Anderb.

*Pseudognaphalium chartaceum (Greenm.) Anderb.

*Pseudognaphalium conoideum (Kunth) Anderb.

*Pseudognaphalium inornatum (DC.) Anderb.

Pseudognaphalium liebmannii (Sch. Bip. ex Klatt) Anderb.

*Pseudognaphalium nubicola (I.M. Johnst.) Anderb.

Pseudognaphalium oxyphyllum (DC.) Kirp.

*Pseudognaphalium purpurascens (DC.) Anderb.

Pseudognaphalium roseum (Kunth) Anderb.

Pseudognaphalium semiamplexicaule (DC.) Anderb.

*Pseudognaphalium semilanatum (DC.) Anderb.

Pseudognaphalium stramineum (Kunth) Anderb.

Pseudognaphalium viscosum (Kunth) Anderb.

Psilactis asteroides A. Gray

Psilactis brevilingulata Sch. Bip. ex Hemsl.

Psilactis gentryi (Stand1.) D.R. Morgan

Pyrrhopappus pauciflorus (D. Don) DC.

Robinsonecio gerberifolius (Sch. Bip.) T.M. Barkley \& J.P. Janovec

*Roldana albonervia (Greenm.) H. Rob. \& Brettell

Roldana angulifolia (DC.) H. Rob. \& Brettell

Roldana aschenborniana (S. Schauer) H. Rob. \& Brettell

Roldana barba-johannis (DC.) H. Rob. \& Brettell

*Roldana chapalensis (S. Watson) H. Rob. \& Brettell

*Roldana hederifolia (Hemsl.) H. Rob. \& Brettell

*Roldana heracleifolia (Hemsl.) H. Rob. \& Brettell

*Roldana hintonii H. Rob. \& Brettell

*Roldana langlassei (Greenm.) H. Rob. \& Brettell 
Apéndice 4: Continuación.

*Roldana lineolata (DC.) H. Rob. \& Brettell

*Roldana lobata La Llave

* Roldana michoacana (B.L. Rob.) H. Rob. \& Brettell

* Roldana platanifolia (Benth.) H. Rob. \& Brettell

* Roldana reticulata (DC.) H. Rob. \& Brettell

* Roldana sessilifolia (Hook. \& Arn.) H. Rob. \& Brettell

* Roldana suffulta (Greenm.) H. Rob. \& Brettell

* Rumfordia floribunda DC.

*Sabazia humilis (Kunth) Cass.

* Sabazia multiradiata (Seaton) Longpre

*Salmea oligocephala Hemsl.

*Salmea palmeri $\mathrm{S}$. Watson

Salmea scandens (L.) DC.

Sanvitalia procumbens Lam.

* Schistocarpha bicolor Less.

Schkuhria pinnata (Lam.) Kuntze ex Thell.

*Schkuhria schkuhrioides (Link. \& Otto) Thell.

Sclerocarpus uniserialis (Hook.) Benth. \& Hook. f. ex Hemsl.

* Selloa plantaginea Kunth

* Senecio argutus Kunth

Senecio callosus Sch. Bip.

* Senecio cinerarioides Kunth

*Senecio deformis Klatt

*Senecio helodes Benth.

*Senecio iodanthus Greenm.

*Senecio jacalensis Greenm.

Senecio mairetianus DC.

*Senecio mulgediifolius S. Schauer

Senecio multidentatus Sch. Bip. ex Hemsl.

*Senecio orizabensis Sch. Bip. ex Hemsl.

* Senecio prionopterus B.L. Rob. \& Greenm.

*Senecio procumbens Kunth

* Senecio roseus Sch. Bip.

* Senecio stoechadiformis DC.

Sigesbeckia agrestis Poepp.

Sigesbeckia jorullensis Kunth

Simsia amplexicaulis (Cav.) Pers.

*Simsia annectens S.F. Blake

Simsia foetida (Cav.) S.F. Blake

Simsia lagasciformis DC.

Simsia sanguinea A. Gray

Sinclairia glabra (Hemsl.) Rydb.

Smallanthus maculatus (Cav.) H. Rob.

* Solidago paniculata DC.

Solidago simplex Kunth

Solidago velutina DC.
* Stevia aschenborniana Sch. Bip.

Stevia caracasana DC.

*Stevia clinopodioides Greenm.

Stevia connata Lag.

Stevia deltoidea Greene

*Stevia dictyophylla B.L. Rob.

Stevia elatior Kunth

* Stevia eupatoria (Spreng.) Willd.

*Stevia hintonii (Grashoff) B.L. Turner

*Stevia hypomalaca B.L. Rob.

*Stevia iltisiana Grashoff

Stevia incognita Grashoff

*Stevia isomeca Grashoff

Stevia jorullensis Kunth

*Stevia latifolia Benth.

Stevia lucida Lag.

Stevia micrantha Lag.

*Stevia monardifolia Kunth

* Stevia nelsonii B.L. Rob.

*Stevia oligophylla Soejima \& Yahara

* Stevia origanoides Kunth

* Stevia orizabensis B.L. Rob.

Stevia ovata Willd.

* Stevia pilosa Lag.

* Stevia porphyrea McVaugh

*Stevia purpusii B.L. Rob.

Stevia salicifolia Cav.

Stevia serrata Cav.

Stevia suaveolens Lag.

*Stevia subpubescens Lag.

* Stevia tephra B.L. Rob.

* Stevia tomentosa Kunth

* Stevia trifida Lag.

Stevia triflora DC.

*Stevia vernicosa Greenm.

Stevia viscida Kunth

*Steviopsis adenosperma (Sch. Bip.) B.L. Turner

*Steviopsis vigintiseta (DC.) R.M. King \& H. Rob.

Symphyotrichum expansum (Poepp. ex Spreng.) G.L. Nesom

Symphyotrichum moranense (Kunth) G.L. Nesom

*Symphyotrichum potosinum (A. Gray) G.L. Nesom

*Tagetes coronopifolia Willd.

Tagetes erecta L.

Tagetes filifolia Lag.

Tagetes foetidissima DC.

Tagetes jaliscensis Greenm. 
Apéndice 4: Continuación.

Tagetes lucida Cav.

*Tagetes lunulata Ortega

Tagetes micrantha Cav.

*Tagetes persicifolius (Benth.) B.L. Turner

*Tagetes pringlei $\mathrm{S}$. Watson

*Tagetes stenophylla B.L. Rob.

Tagetes subulata Cerv.

Tagetes tenuifolia Cav.

* Telanthophora andrieuxii (DC.) H. Rob. \& Brettell

Thymophylla pentachaeta (DC.) Small

*Thymophylla tenuifolia (Cass.) Rydb.

Tithonia diversifolia (Hemsl.) A. Gray

Tithonia rotundifolia (Mill.) S.F. Blake

Tithonia tubiformis (Jacq.) Cass.

*Trichocoronis sessilifolia (S. Schauer) B.L. Rob.

*Tridax balbisioides (Kunth) A. Gray

*Tridax brachylepis Hemsl.

*Tridax coronopifolia (Kunth) Hemsl.

*Tridax mexicana A.M. Powell

*Tridax palmeri A. Gray

Tridax platyphylla B.L. Rob.

Tridax procumbens $\mathrm{L}$.

*Tridax rosea Sch. Bip. ex B.L. Rob. \& Greenm.

*Tridax trilobata (Cav.) Hemsl.

Trigonospermum annuum McVaugh \& Lask.

Trigonospermum melampodioides DC.

*Trixis alata D. Don

Trixis inula Crantz

*Trixis megalophylla Greenm.

*Trixis mexicana Lex.

*Trixis michuacana Lex.

*Trixis pringlei B.L. Rob. \& Greenm.

*Verbesina abscondita Klatt

*Verbesina angustifolia (Benth.) S.F. Blake

Verbesina crocata (Cav.) Less.

* Verbesina fastigiata B.L. Rob. \& Greenm.

*Verbesina grayii (Sch. Bip.) Benth. ex Hemsl.

*Verbesina hypomalaca B.L. Rob. \& Greenm.

*Verbesina klattii B.L. Rob. \& Greenm.

*Verbesina mexiae B.L. Turner

*Verbesina oncophora B.L. Rob. \& Seaton

*Verbesina ovata (Cav.) A. Gray

*Verbesina parviflora (Kunth) S.F. Blake

*Verbesina pedunculosa (DC.) B.L. Rob.

*Verbesina seatonii S.F. Blake

*Verbesina serrata Cav.
*Verbesina sphaerocephala A. Gray

*Verbesina tetraptera (Ortega) A. Gray

*Verbesina virgata Cav.

*Vernonanthura cordata (Kunth) H. Rob.

*Vernonanthura liatroides (DC.) H. Rob.

Vernonanthura patens (Kunth) H. Rob.

*Vernonia alamanii DC.

Viguiera dentata (Cav.) Spreng.

**Viguiera sultepecana Paray

*Wamalchitamia strigosa (DC.) Strother

Wedelia acapulcensis Kunth

*Wedelia hintoniorum B.L. Turner

Xanthisma gymnocephalum (DC.) D.R. Morgan \& R.L. Hartm.

Xanthium strumarium L.

*Xanthocephalum benthamianum Hemsl.

*Xanthocephalum centauroides Willd.

*Xanthocephalum humile (Kunth) Benth. \& Hook. f.

*Zaluzania augusta (Lag.) Sch. Bip.

*Zaluzania megacephala Sch. Bip.

*Zaluzania montagnifolia (Sch. Bip.) Sch. Bip.

*Zaluzania triloba (Ortega) Pers.

*Zandera andersoniae (B.L. Turner) D.L. Schulz

Zinnia americana (Mill.) Olorode \& A.M. Torres

*Zinnia angustifolia Kunth

Zinnia elegans Jacq.

*Zinnia haageana Regel

Zinnia peruviana (L.) L.

*Zinnia zinnioides (Kunth) Olorode \& A.M. Torres

\section{Basellaceae}

Anredera vesicaria (Lam.) C.F. Gaertn.

\section{Begoniaceae}

*Begonia asteroides L.B. Sm. \& B.G. Schub.

*Begonia balmisiana Ruiz ex Klotzsch

Begonia biserrata Lindl.

* Begonia cristobalensis Ziesenh.

*Begonia cylindrata L.B. Sm. \& B.G. Schub.

* Begonia falciloba Liebm.

Begonia fusca Liebm.

Begonia gracilis Kunth

Begonia heracleifolia Schltdl. \& Cham.

*Begonia hintoniana L.B. Sm. \& B.G. Schub.

* Begonia incarnata Link \& Otto

Begonia manicata Brongn. ex F. Cels

*Begonia monophylla A. DC. 
Apéndice 4: Continuación.

Begonia oaxacana A. DC.

* Begonia ornithocarpa Standl.

Begonia plebeja Liebm.

* Begonia squarrosa Liebm.

Begonia stigmosa Lindl.

\section{Berberidaceae}

* Berberis alpina Zamudio

Berberis moranensis Schult. \& Schult. f.

\section{Betulaceae}

Alnus acuminata Kunth

Alnus jorullensis Kunth

Carpinus tropicalis (Donn. Sm.) Lundell

Ostrya virginiana (Mill.) K. Koch

\section{Bignoniaceae}

Adenocalymma inundatum Mart. ex DC.

Amphilophium crucigerum (L.) L.G. Lohmann

Amphilophium paniculatum (L.) Kunth

Astianthus viminalis (Kunth) Baill.

Crescentia alata Kunth

Dolichandra unguis-cati (L.) L.G. Lohmann

Fridericia dichotoma (Jacq.) L.G. Lohmann

Fridericia patellifera (Schltdl.) L.G. Lohmann

Godmania aesculifolia (Kunth) Standl.

Handroanthus impetiginosus (Mart. ex DC.) Mattos

Mansoa hymenaea (DC.) A.H. Gentry

Parmentiera aculeata (Kunth) Seem.

Tabebuia rosea (Bertol.) A. DC.

Tecoma stans (L.) Juss. ex Kunth

\section{Bixaceae}

Cochlospermum vitifolium (Willd.) Spreng.

\section{Boraginaceae}

*Antiphytum parryi S. Watson

*Bourreria andrieuxii (DC.) Hemsl.

Bourreria huanita (Lex.) Hemsl.

*Cordia elaeagnoides DC.

* Cordia morelosana Standl.

Cordia salvadorensis Standl.

*Cordia tinifolia Willd. ex Roem. \& Schult.

Cryptantha albida (Kunth) I.M. Johnst.

Ehretia latifolia DC.

Hackelia mexicana (Schltdl. \& Cham.) I.M. Johnst.
Heliotropium angiospermum Murray

Heliotropium curassavicum L.

Heliotropium fallax I.M. Johnst.

Heliotropium foliosissimum J.F. Macbr.

Heliotropium fruticosum $\mathrm{L}$.

Heliotropium indicum $\mathrm{L}$.

Heliotropium pringlei B.L. Rob.

Heliotropium procumbens Mill.

Lennoa madreporoides Lex.

Lithospermum calycosum (J.F. Macbr.) I.M. Johnst.

*Lithospermum discolor M. Martens \& Galeotti

Lithospermum distichum Ortega

*Lithospermum exsertum (D. Don) J.I. Cohen

*Lithospermum oblongifolium Greenm.

Lithospermum pringlei I.M. Johnst.

*Lithospermum rzedowskii J.I. Cohen

*Lithospermum strictum Lehm.

*Lithospermum trinervium (Lehm.) J.I. Cohen

Nama dichotoma (Ruiz \& Pav.) Choisy

* Nama origanifolia Kunth

* Nama prostrata Brand

Nama undulata Kunth

*Phacelia coulteri Greenm.

Phacelia heterophylla Pursh

Phacelia platycarpa (Cav.) Spreng.

Tournefortia acutiflora M. Martens \& Galeotti

* Tournefortia calycina Benth.

Tournefortia glabra L.

*Tournefortia hartwegiana Steud.

Tournefortia hirsutissima L.

Tournefortia mutabilis Vent.

Tournefortia petiolaris DC.

Tournefortia volubilis $\mathrm{L}$.

Varronia curassavica Jacq.

Varronia globosa Jacq.

Varronia inermis (Mill.) Borhidi

Wigandia urens (Ruiz \& Pav.) Kunth

\section{Brassicaceae}

Cardamine bonariensis Pers.

Cardamine obliqua Hochst. ex A. Rich.

*Chaunanthus petiolatus (Hemsl.) O.E. Schulz

Descurainia impatiens (Cham. \& Schltdl.) O.E. Schulz

*Descurainia virletii (E. Fourn.) O.E. Schulz

Draba jorullensis Kunth

* Draba nivicola Rose 
Apéndice 4: Continuación.

Erysimum asperum (Nutt.) DC.

*Erysimum macradenium J. Gay

*Exhalimolobos berlandieri (E. Fourn.) Al-Shehbaz \& C.D. Bailey

Exhalimolobos hispidulus (DC.) Al-Shehbaz \& C.D. Bailey

*Exhalimolobos polyspermus (E. Fourn.) Al-Shehbaz \& C.D. Bailey

Hesperidanthus linearifolius (A. Gray) Rydb.

Lepidium bipinnatifidum Desv.

Lepidium oblongum Small

*Lepidium schaffneri Thell.

Lepidium sordidum A. Gray

Lepidium virginicum $\mathrm{L}$.

Nasturtium officinale $\mathrm{R}$. Br.

Pennellia longifolia (Benth.) Rollins

Pennellia micrantha (A. Gray) Nieuwl.

*Pennellia patens (O.E. Schulz) Rollins

Romanschulzia arabiformis (DC.) Rollins

Rorippa mexicana (Moc. \& Sessé ex DC.) Standl. \& Steyerm.

Rorippa pinnata (Sessé \& Moc.) Rollins

\section{Brunelliaceae}

Brunellia mexicana Standl.

\section{Burseraceae}

* Bursera ariensis (Kunth) McVaugh \& Rzed.

* Bursera aspleniifolia Brandegee

*Bursera bicolor (Willd. ex Schltdl.) Engl.

Bursera bipinnata (DC.) Engl.

* Bursera confusa (Rose) Engl.

* Bursera copallifera (Sessé \& Moc. ex DC.) Bullock

* Bursera coyucensis Bullock

*Bursera cuneata (Schltdl.) Engl.

* Bursera discolor Rzed.

Bursera diversifolia Rose

Bursera excelsa (Kunth) Engl.

Bursera fagaroides (Kunth) Engl.

*Bursera glabrifolia (Kunth) Engl.

*Bursera grandifolia (Schltdl.) Engl.

Bursera graveolens (Kunth) Triana \& Planch.

Bursera heteresthes Bullock

*Bursera hintonii Bullock

*Bursera kerberi Engl.

*Bursera lancifolia (Schltdl.) Engl.

* Bursera linanoe (La Llave) Rzed., Calderón \& Medina

*Bursera longipes (Rose) Standl.

* Bursera mirandae C.A. Toledo

Bursera morelensis Ramírez
Bursera ovalifolia (Schltdl.) Engl.

*Bursera penicillata (Sessé \& Moc. ex DC.) Engl.

Bursera simaruba (L.) Sarg.

* Bursera submoniliformis Engl.

* Bursera trifoliolata Bullock

* Bursera trimera Bullock

*Bursera velutina Bullock

\section{Cactaceae}

*Coryphantha elephantidens (Lem.) Lem.

*Coryphantha ottonis (Pfeiff.) Lem.

*Coryphantha pycnacantha (Mart.) Lem.

Cylindropuntia imbricata (Haw.) F.M. Knuth

Cylindropuntia rosea (DC.) Backeb.

Cylindropuntia tunicata (Lehm.) F.M. Knuth

*Disocactus flagelliformis (L.) Barthlott

Disocactus speciosus (Cav.) Barthlott

Echinocactus horizonthalonius Lem.

Echinocereus cinerascens (DC.) Lem.

*Epiphyllum anguliger (Lem.) G. Don

*Ferocactus latispinus (Haw.) Britton \& Rose

Hylocereus ocamponis (Salm-Dyck) Britton \& Rose

*Hylocereus purpusii (Weing.) Britton \& Rose

Hylocereus undatus (Haw.) Britton \& Rose

*Lophocereus marginatus (DC.) S. Arias \& Terrazas

*Mammillaria backebergiana Buchenau

* Mammillaria beneckei Ehrenb.

Mammillaria compressa DC.

* Mammillaria discolor Haw.

* Mammillaria haageana Pfeiff.

* Mammillaria karwinskiana Mart.

* Mammillaria magnimamma Haw.

*Mammillaria matudae Bravo

* Mammillaria meyranii Bravo

*Mammillaria nunezii (Britton \& Rose) Orcutt

* Mammillaria rhodantha Link \& Otto

*Mammillaria spinosissima Lem.

* Mammillaria uncinata Zucc. ex Pfeiff.

* Mammillaria wiesingeri Boed.

* Mammillaria zephyranthoides Scheidw.

*Myrtillocactus geometrizans (Mart. ex Pfeiff.) Console

*Neobuxbaumia mezcalaensis (Bravo) Backeb.

*Nyctocereus serpentinus (Lag. \& Rodr.) Britton \& Rose

* Opuntia atropes Rose

* Opuntia auberi Pfeiff.

* Opuntia cochinera Griffiths 
Apéndice 4: Continuación.

Opuntia decumbens Salm-Dyck

Opuntia engelmannii Salm-Dyck

* Opuntia fuliginosa Griffiths

Opuntia guilanchi Griffiths

* Opuntia hyptiacantha F.A.C. Weber

* Opuntia joconostle F.A.C. Weber

* Opuntia karwinskiana Salm-Dyck

* Opuntia lasiacantha Pfeiff.

Opuntia megacantha Salm-Dyck

Opuntia pubescens J.C. Wendl. ex Pfeiff.

Opuntia robusta $\mathrm{H}$. Wendl.

* Opuntia spinulifera Salm-Dyck

* Opuntia streptacantha Lem.

* Opuntia tomentosa Salm-Dyck

* Opuntia velutina F.A.C. Weber

*Pachycereus grandis Rose

*Peniocereus maculatus (Weing.) Cutak

*Pereskiopsis diguetii (F.A.C. Weber) Britton \& Rose

*Pereskiopsis rotundifolia (DC.) Britton \& Rose

*Pilosocereus alensis (F.A.C. Weber) Byles \& G.D. Rowley

*Stenocactus anfractuosus (Mart. ex Pfeiff.) A. Berger ex A.W. Hill

*Stenocactus crispatus (DC.) A. Berger ex A.W. Hill

*Stenocactus dichroacanthus (Mart. ex Pfeiff.) A. Berger ex Backeb. \& F.M. Knuth

*Stenocactus heteracanthus (Muehlenpf.) A. Berger ex A.W. Hill

*Stenocactus obvallatus (DC.) A. Berger ex A.W. Hill

*Stenocactus phyllacanthus (Mart. ex A. Dietr. \& Otto) A. Berger ex A.W. Hill

*Stenocereus beneckei (Ehrenb.) Buxb.

*Stenocereus dumortieri (Scheidw.) Buxb.

*Stenocereus queretaroensis (F.A.C. Weber) Buxb.

\section{Calceolariaceae}

Calceolaria mexicana Benth.

Calceolaria tripartita Ruiz \& Pav.

\section{Campanulaceae}

Calcaratolobelia cordifolia (Hook. \& Arn.) Wilbur

* Calcaratolobelia tenella (Turcz.) Wilbur

Diastatea micrantha (Kunth) McVaugh

Diastatea tenera (A. Gray) McVaugh

Lobelia berlandieri A. DC.

Lobelia cardinalis L.

Lobelia cliffortiana L.

Lobelia fenestralis Cav.

*Lobelia gruina Cav.
Lobelia irasuensis Planch. \& Oerst.

Lobelia laxiflora Kunth

Lobelia longicaulis Brandegee

Lobelia nana Kunth

Triodanis perfoliata (L.) Nieuwl.

\section{Cannabaceae}

Aphananthe monoica (Hemsl.) J.-F. Leroy

Celtis caudata Planch.

Celtis iguanaea (Jacq.) Sarg.

Trema micrantha (L.) Blume

\section{Capparaceae}

*Forchhammeria pallida Liebm.

\section{Caprifoliaceae}

*Lonicera mexicana (Kunth) Rehder

*Lonicera pilosa (Kunth) Willd. ex Kunth

Symphoricarpos microphyllus Kunth

*Valeriana ceratophylla Kunth

Valeriana clematitis Kunth

*Valeriana densiflora Benth.

Valeriana edulis Nutt.

*Valeriana laciniosa M. Martens \& Galeotti

*Valeriana naidae Barrie

*Valeriana palmeri A. Gray

*Valeriana pratensis (Benth.) Steud.

Valeriana robertianifolia Briq.

*Valeriana rzedowskiorum Barrie

Valeriana scandens L.

*Valeriana selerorum Graebn. \& Loesen.

Valeriana sorbifolia Kunth

Valeriana urticifolia Kunth

*Valeriana vaginata Kunth

\section{Caricaceae}

Carica papaya L.

Jacaratia mexicana A. DC.

*Jarilla heterophylla (Cerv. ex La Llave) Rusby

\section{Caryophyllaceae}

Arenaria bourgaei Hemsl.

Arenaria bryoides Willd. ex D.F.K. Schltdl.

Arenaria lanuginosa (Michx.) Rohrb.

Arenaria lycopodioides Willd. ex D.F.K. Schltdl.

*Arenaria oresbia Greenm. 
Apéndice 4: Continuación.

Arenaria paludicola B.L. Rob.

Arenaria reptans Hemsl.

Cardionema ramosissimum (Weinm.) A. Nelson \& J.F. Macbr.

Cerastium brachypodum (Engelm. ex A. Gray) B.L. Rob. ex Britton

Cerastium nutans Raf.

*Cerastium orithales Schltdl.

* Cerastium purpusii Greenm.

* Cerastium ramigerum Bartl.

*Cerastium tolucense D.A. Good

Cerastium vulcanicum Schltdl.

*Cerdia virescens Moc. \& Sessé ex DC.

Colobanthus quitensis (Kunth) Bartl.

Corrigiola andina Planch. \& Triana

Drymaria arenarioides Humb. \& Bonpl. ex Roem. \& Schult.

Drymaria cordata (L.) Willd. ex Roem. \& Schult.

Drymaria effusa A. Gray

*Drymaria excisa Standl.

Drymaria glandulosa Bartl.

Drymaria gracilis Schltdl. \& Cham.

Drymaria laxiflora Benth.

Drymaria leptophylla (Cham. \& Schltdl.) Fenzl ex Rohrb.

*Drymaria malachioides Briq.

Drymaria molluginea (Ser.) Didr.

Drymaria multiflora Brandegee

* Drymaria tenuis S. Watson

Drymaria villosa Schltdl. \& Cham.

*Drymaria xerophylla A. Gray

*Lychnis mexicana Rose

*Minuartia moehringioides (Moc. \& Sessé ex DC.) Mattf.

* Paronychia mexicana Hemsl.

Sagina procumbens L.

Sagina saginoides (L.) H. Karst.

* Scopulophila parryi (Hemsl.) I.M. Johnst.

Silene laciniata Cav.

* Spergularia mexicana Hemsl.

Stellaria cuspidata Willd. ex Schltdl.

Stellaria umbellata Turcz.

\section{Celastraceae}

*Celastrus pringlei Rose

Hippocratea volubilis $\mathrm{L}$.

Pristimera celastroides (Kunth) A.C. Sm.

Semialarium mexicanum (Miers) Mennega

*Wimmeria lanceolata Rose

*Wimmeria persicifolia Radlk.

*Zinowiewia concinna Lundell

Zinowiewia integerrima (Turcz.) Turcz.

\section{Chrysobalanaceae}

Licania arborea Seem.

\section{Cistaceae}

*Helianthemum concolor (L. Riley) J.G. Ortega

Helianthemum coulteri S. Watson

Helianthemum glomeratum (Lag.) Lag. ex Dunal

*Helianthemum patens Hemsl.

Lechea tripetala (Moc. \& Sessé ex Dunal) Britton

\section{Cleomaceae}

Cleoserrata speciosa (Raf.) H.H. Iltis

Peritoma multicaulis (DC.) H.H. Iltis

Polanisia uniglandulosa (Cav.) DC.

\section{Clethraceae}

* Clethra hartwegii Britton

Clethra lanata M. Martens \& Galeotti

Clethra mexicana DC.

* Clethra pringlei $\mathrm{S}$. Watson

*Clethra rosei Britton

\section{Clusiaceae}

Clusia massoniana Lundell

Clusia salvinii Donn. Sm.

\section{Combretaceae}

Combretum argenteum Bertol.

Combretum fruticosum (Loefl.) Stuntz

\section{Convolvulaceae}

Bonamia sulphurea (Brandegee) Myint \& D.B. Ward

*Calycobolus nutans (Moc. \& Sessé ex Choisy) D.F. Austin

Convolvulus equitans Benth.

Convolvulus nodiflorus Desr.

Cuscuta americana $\mathrm{L}$.

Cuscuta applanata Engelm.

Cuscuta corymbosa Ruiz \& Pav.

*Cuscuta gracillima Engelm.

Cuscuta mitriformis Engelm. ex Hemsl.

Cuscuta obtusiflora Kunth

*Cuscuta potosina W. Schaffn. ex Engelm.

Cuscuta rugosiceps Yunck.

Cuscuta tinctoria Mart. ex Engelm.

Cuscuta umbellata Kunth

Dichondra argentea Humb. \& Bonpl. ex Willd. 
Apéndice 4: Continuación.

Dichondra sericea Sw.

Evolvulus alsinoides (L.) L.

*Evolvulus prostratus B.L. Rob.

Evolvulus sericeus $\mathrm{Sw}$.

Evolvulus tenuis Mart. ex Choisy

Ipomoea alba $\mathrm{L}$.

* Ipomoea ampullacea Fernald

Ipomoea arborescens (Humb. \& Bonpl. ex Willd.) G. Don

Ipomoea aristolochiifolia $\mathrm{G}$. Don

Ipomoea batatas (L.) Lam.

*Ipomoea bracteata Cav.

Ipomoea capillacea (Kunth) G. Don

Ipomoea carnea Jacq.

Ipomoea cholulensis Kunth

*Ipomoea conzattii Greenm.

Ipomoea costellata Torr.

Ipomoea cristulata Hallier f.

* Ipomoea dimorphophylla Greenm.

Ipomoea dumetorum Willd. ex Roem. \& Schult.

Ipomoea dumosa (Benth.) L.O. Williams

Ipomoea elongata Choisy

*Ipomoea emetica Choisy

*Ipomoea funis Schltdl. \& Cham.

Ipomoea hederifolia L.

*Ipomoea igualensis Weath.

Ipomoea indica (Burm.) Merr.

*Ipomoea jalapa (L.) Pursh

Ipomoea lindenii M. Martens \& Galeotti

Ipomoea lobata (Cerv.) Thell.

*Ipomoea madrensis S. Watson

Ipomoea mairetii Choisy

Ipomoea meyeri (Spreng.) G. Don

Ipomoea microsepala Benth.

Ipomoea muricata (L.) Jacq.

Ipomoea murucoides Roem. \& Schult.

Ipomoea neei (Spreng.) O’Donell

Ipomoea nil (L.) Roth

Ipomoea oocarpa Benth.

Ipomoea orizabensis (G. Pelletan) Ledeb. ex Steud.

Ipomoea pauciflora $\mathrm{M}$. Martens \& Galeotti

Ipomoea populina House

Ipomoea praecana House

Ipomoea pubescens Lam.

Ipomoea purga (Wender.) Hayne

Ipomoea purpurea (L.) Roth

*Ipomoea robinsonii House
Ipomoea santillanii O’Donell

*Ipomoea simulans D. Hanb.

*Ipomoea stans Cav.

*Ipomoea suaveolens (M. Martens \& Galeotti) Hemsl.

Ipomoea suffulta (Kunth) G. Don

Ipomoea ternifolia Cav.

Ipomoea tricolor Cav.

Ipomoea trifida (Kunth) G. Don

Ipomoea triloba $\mathrm{L}$.

Ipomoea wolcottiana Rose

Jacquemontia pentanthos (Jacq.) G. Don

Jacquemontia sphaerostigma (Cav.) Rusby

Jacquemontia tamnifolia (L.) Griseb.

Merremia aegyptia (L.) Urb.

Merremia cissoides (Lam.) Hallier f.

Merremia dissecta (Jacq.) Hallier f.

Merremia quinquefolia (L.) Hallier f.

Merremia umbellata (L.) Hallier f.

Operculina pinnatifida (Kunth) O'Donell

Operculina pteripes (G. Don) O’Donell

Turbina corymbosa (L.) Raf.

\section{Coriariaceae}

Coriaria ruscifolia L.

\section{Cornaceae}

Cornus disciflora Moc. \& Sessé ex DC.

Cornus excelsa Kunth

\section{Crassulaceae}

Crassula aquatica (L.) Schönland

* Echeveria coccinea (Cav.) DC.

* Echeveria fulgens Lem.

Echeveria gibbiflora DC.

*Echeveria goldmanii Rose

*Echeveria grandiflora Haw.

* Echeveria mucronata Schltdl.

* Echeveria obtusifolia Rose

* Echeveria secunda Booth ex Lindl.

*Echeveria subrigida (B.L. Rob. \& Seaton) Rose

**Echeveria valvata Moran

*Echeveria waltheri Moran \& J. Meyrán

*Sedum bourgaei Hemsl.

*Sedum calcaratum Rose

**Sedum clavatum R.T. Clausen

* Sedum clavifolium Rose 
Apéndice 4: Continuación.

**Sedum cormiferum R.T. Clausen

* Sedum dendroideum Moc. \& Sessé ex DC.

Sedum ebracteatum Moc. \& Sessé ex DC.

*Sedum frutescens Rose

Sedum goldmanii (Rose) Moran

*Sedum greggii Hemsl.

* Sedum hemsleyanum Rose

*Sedum jaliscanum $\mathrm{S}$. Watson

* Sedum jurgensenii (Hemsl.) Moran

* Sedum longipes Rose

*Sedum minimum Rose

Sedum moranense Kunth

*Sedum napiferum Peyr.

**Sedum ocuilense J. Meyrán

*Sedum oxypetalum Kunth

**Sedum pentastamineum R.T. Clausen

Sedum praealtum A. DC.

*Sedum quevae Raym.-Hamet

**Sedum tehuaztlense Moran \& J. Meyrán

*Sedum tortuosum Hemsl.

*Sedum versadense C.H. Thomps.

*Villadia misera (Lindl.) R.T. Clausen

\section{Cucurbitaceae}

Cayaponia attenuata (Hook. \& Arn.) Cogn. Cionosicyos macranthus (Pittier) C. Jeffrey

Cucurbita ficifolia Bouché

Cucurbita foetidissima Kunth

Cucurbita pepo L.

*Cucurbita radicans Naudin

Cyclanthera dioscoreoides C.E. Jones \& Kearns

Cyclanthera gracillima Cogn.

Cyclanthera integrifoliola Cogn.

Cyclanthera langaei Cogn.

Cyclanthera multifoliola Cogn.

Cyclanthera ribiflora (Schltdl.) Cogn.

* Cyclanthera rostrata (Paul G. Wilson) Kearns \& C.E. Jones

*Cyclanthera tamnoides (Willd.) Cogn.

*Echinopepon cirrhopedunculatus Rose

Echinopepon coulteri (A. Gray) Rose

*Echinopepon milleflorus Naudin

Echinopepon paniculatus (Cogn.) Dieterle

Echinopepon racemosus (Steud.) C. Jeffrey

Echinopepon torquatus (Ser.) Rose

Melothria pendula L.

*Melothria pringlei (S. Watson) Mart. Crov.
Microsechium palmatum (Ser.) Cogn.

Polyclathra albiflora (Cogn.) C. Jeffrey

Rytidostylis gracilis Hook. \& Arn.

*Rytidostylis longisepala (Cogn.) C. Jeffrey

*Schizocarpum parviflorum B.L. Rob. \& Greenm.

*Schizocarpum reflexum Rose

*Sechiopsis triquetra (Moc. \& Sessé ex Ser.) Naudin

Sechium compositum (Donn. Sm.) C. Jeffrey

Sechium edule (Jacq.) Sw.

*Sechium hintonii (Paul G. Wilson) C. Jeffrey

Sicyos laciniatus L.

Sicyos longisepalus Cogn.

*Sicyos microphyllus Kunth

Sicyos parviflorus Willd.

\section{Cytinaceae}

Bdallophytum americanum (R. Br.) Eichler ex Solms

\section{Dilleniaceae}

Curatella americana $\mathrm{L}$.

\section{Dipentodontaceae}

Perrottetia longistylis Rose

\section{Ebenaceae}

*Diospyros oaxacana Standl.

Diospyros salicifolia Humb. \& Bonpl. ex Willd.

\section{Elatinaceae}

Elatine brachysperma A. Gray

\section{Ericaceae}

Agarista mexicana (Hemsl.) Judd

Arbutus arizonica (A. Gray) Sarg.

*Arbutus bicolor S. González, M. González \& P.D. Sørensen

*Arbutus tessellata P.D. Sørensen

Arbutus xalapensis Kunth

Arctostaphylos pungens Kunth

Chimaphila maculata (L.) Pursh

Chimaphila umbellata (L.) W.P.C. Barton

Comarostaphylis discolor (Hook.) Diggs

*Comarostaphylis glaucescens (Kunth) Zucc. ex Klotzsch

*Comarostaphylis longifolia (Benth.) Klotzsch

*Comarostaphylis polifolia (Kunth) Zucc. ex Klotzsch

* Gaultheria angustifolia Brandegee

Gaultheria erecta Vent. 
Apéndice 4: Continuación.

Monotropa hypopitys L.

Monotropa uniflora L.

Orthilia secunda (L.) House

Pernettya ciliata (Schltdl. \& Cham.) Small

Pernettya prostrata (Cav.) DC.

Pterospora andromedea Nutt.

Pyrola angustifolia (Alef.) Hemsl.

Vaccinium confertum Kunth

Vaccinium geminiflorum Kunth

Vaccinium leucanthum Schltdl.

\section{Erythroxylaceae}

*Erythroxylum mexicanum Kunth

\section{Euphorbiaceae}

Acalypha adenostachya Müll. Arg.

Acalypha alopecuroidea Jacq.

Acalypha arvensis Poepp. \& Endl.

* Acalypha botteriana Müll. Arg.

Acalypha macrostachya Jacq.

Acalypha mexicana Müll. Arg.

Acalypha mollis Kunth

Acalypha persimilis Müll. Arg.

Acalypha phleoides Cav.

Acalypha schiedeana Schltdl.

Acalypha setosa A. Rich.

**Acalypha subtomentosa Lag.

Acalypha triloba Müll. Arg.

Caperonia palustris (L.) A. St.-Hil.

Cnidoscolus aconitifolius (Mill.) I.M. Johnst.

Cnidoscolus angustidens Torr.

Cnidoscolus multilobus (Pax) I.M. Johnst.

*Cnidoscolus rostratus Lundell

Cnidoscolus urens (L.) Arthur

Croton adspersus Benth.

Croton ciliatoglandulifer Ortega

Croton conspurcatus Schltdl.

Croton dioicus Cav.

*Croton ehrenbergii Schltdl.

Croton morifolius Willd.

* Dalembertia populifolia Baill.

*Ditaxis pringlei (Greenm.) Pax \& K. Hoffm.

Euphorbia alta Norton

Euphorbia anychioides Boiss.

Euphorbia ariensis Kunth

*Euphorbia bracteata Jacq.
* Euphorbia calyculata Kunth

Euphorbia chamaesula Boiss.

Euphorbia cotinifolia L.

Euphorbia cyathophora Murray

Euphorbia delicatula Boiss.

Euphorbia densiflora (Klotzsch \& Garcke) Klotzsch

Euphorbia dentata Michx.

*Euphorbia dioscoreoides Boiss.

*Euphorbia eglandulosa V.W. Steinm.

Euphorbia francoana Boiss.

Euphorbia furcillata Kunth

Euphorbia graminea Jacq.

Euphorbia heterophylla L.

*Euphorbia hintonii L.C. Wheeler

Euphorbia hirta L.

Euphorbia hypericifolia L.

Euphorbia hyssopifolia L.

Euphorbia indivisa (Engelm.) Tidestr.

* Euphorbia lacera Boiss.

Euphorbia macropus (Klotzsch \& Garcke) Boiss.

Euphorbia mendezii Boiss.

*Euphorbia misella S. Watson

Euphorbia multiseta Benth.

Euphorbia nutans Lag.

Euphorbia ocymoidea L.

Euphorbia ophthalmica Pers.

*Euphorbia potosina Fernald

Euphorbia prostrata Aiton

Euphorbia pulcherrima Willd. ex Klotzsch

Euphorbia radians Benth.

Euphorbia schiedeana (Klotzsch \& Garcke) Mayfield ex C. Nelson

Euphorbia schlechtendalii Boiss.

Euphorbia serpens Kunth

Euphorbia serpyllifolia Pers.

Euphorbia sonorae Rose

*Euphorbia sphaerorhiza Benth.

Euphorbia stictospora Engelm.

* Euphorbia subreniformis S. Watson

* Euphorbia tanquahuete Sessé \& Moc.

Euphorbia tithymaloides L.

Euphorbia villifera Scheele

Hura polyandra Baill.

*Jatropha ciliata Sessé ex Cerv.

Jatropha curcas L.

Jatropha dioica Sessé ex Cerv.

Manihot angustiloba (Torr.) Müll. Arg. 
Apéndice 4: Continuación.

Manihot esculenta Crantz

Manihot rhomboidea Müll. Arg.

*Manihot triloba (Sessé ex Cerv.) McVaugh ex Miranda

Sapium lateriflorum Hemsl.

Sapium macrocarpum Müll. Arg.

Stillingia zelayensis (Kunth) Müll. Arg.

*Tragia affinis B.L. Rob. \& Greenm.

Tragia nepetifolia Cav.

Tragia volubilis L.

\section{Fabaceae}

Acaciella angustissima (Mill.) Britton \& Rose

*Acaciella hartwegii (Benth.) Britton \& Rose

* Acaciella igualensis Britton \& Rose

* Acaciella painteri Britton \& Rose

* Acaciella tequilana (S. Watson) Britton \& Rose

Acaciella villosa (Sw.) Britton \& Rose

Aeschynomene americana $\mathrm{L}$.

Aeschynomene fascicularis Schltdl. \& Cham.

* Aeschynomene hintonii Sandwith

Aeschynomene histrix Poir.

Aeschynomene paniculata Willd. ex Vogel

*Aeschynomene paucifoliolata Micheli

*Aeschynomene petraea B.L. Rob.

Aeschynomene rudis Benth.

Aeschynomene scabra G. Don

Aeschynomene villosa Poir.

Albizia tomentosa (Micheli) Standl.

*Amicia zygomeris DC.

Andira inermis (W. Wright) Kunth ex DC.

*Astragalus diphacus S. Watson

*Astragalus esperanzae M.E. Jones

Astragalus guatemalensis Hemsl.

*Astragalus hidalgensis (Rydb.) Barneby

*Astragalus hypoleucus S. Schauer

*Astragalus lyonnetii Barneby

*Astragalus micranthus Desv.

Astragalus mollissimus Torr.

Astragalus nuttallianus DC.

*Astragalus oxyrhynchus Hemsl.

*Astragalus quinqueflorus S. Watson

* Astragalus radicans Hornem.

*Astragalus strigulosus Kunth

*Astragalus tolucanus B.L. Rob. \& Seaton

Astragalus wootonii E. Sheld.

*Ateleia arsenii Standl.
Bauhinia divaricata $\mathrm{L}$.

Bauhinia pauletia Pers.

*Bauhinia pringlei $\mathrm{S}$. Watson

Bauhinia ungulata $\mathrm{L}$.

*Brongniartia cuneata L.B. Sm. \& B.G. Schub.

* Brongniartia discolor Brandegee

* Brongniartia foliolosa Benth. ex Hemsl.

* Brongniartia funiculata L.B. Sm. \& B.G. Schub.

*Brongniartia intermedia Moric.

*Brongniartia lupinoides (Kunth) Taub.

*Brongniartia mollis Kunth

*Brongniartia podalyrioides Kunth

* Brongniartia pringlei Rydb.

Calliandra eriophylla Benth.

Calliandra grandiflora (L'Hér.) Benth.

Calliandra houstoniana (Mill.) Standl.

Calliandra humilis Benth.

Calopogonium caeruleum (Benth.) C. Wright

Canavalia brasiliensis Mart. ex Benth.

Canavalia hirsutissima J.D. Sauer

Canavalia villosa Benth.

Cassia grandis L. f.

* Cassia hintonii Sandwith

Centrosema plumieri (Turpin ex Pers.) Benth.

Centrosema pubescens Benth.

Centrosema virginianum (L.) Benth.

Chamaecrista nictitans (L.) Moench

Chamaecrista rotundifolia (Pers.) Greene

Clitoria mexicana Link

Cologania angustifolia Kunth

Cologania biloba (Lindl.) G. Nicholson

Cologania broussonetii (Balb.) DC.

Cologania glabrior Rose

Cologania obovata Schltdl.

Cologania procumbens Kunth

Cologania rufescens Rose

*Conzattia multiflora (B.L. Rob.) Standl.

Coursetia glandulosa A. Gray

* Coursetia hidalgoana Lavin

*Coursetia madrensis Micheli

*Coursetia mollis B.L. Rob. \& Greenm.

Coursetia pumila (Rose) Lavin

*Crotalaria bupleurifolia Cham. \& Schltdl.

Crotalaria cajanifolia Kunth

* Crotalaria filifolia Rose

Crotalaria incana L. 
Apéndice 4: Continuación.

Crotalaria longirostrata Hook. \& Arn.

Crotalaria maypurensis Kunth

Crotalaria micans Link

Crotalaria mollicula Kunth

*Crotalaria polyphylla L. Riley

Crotalaria pumila Ortega

Crotalaria rotundifolia J.F. Gmel.

* Crotalaria rzedowskii J. Espinosa

Crotalaria sagittalis L.

Crotalaria vitellina Ker Gawl.

* Dalbergia congestiflora Pittier

* Dalbergia glomerata Hemsl.

*Dalea aenigma Barneby

Dalea alopecuroides Willd.

Dalea bicolor Humb. \& Bonpl. ex Willd.

Dalea brachystachys A. Gray

Dalea citriodora Willd.

Dalea cliffortiana Willd.

* Dalea elata Hook. \& Arn.

* Dalea filiciformis B.L. Rob. \& Greenm.

Dalea foliolosa (Aiton) Barneby

*Dalea hegewischiana Steud.

Dalea humilis G. Don

Dalea leporina (Aiton) Bullock

*Dalea leucostachya A. Gray

Dalea lutea (Cav.) Willd.

* Dalea minutifolia (Rydb.) Harms

*Dalea mucronata DC.

Dalea nutans (Cav.) Willd.

Dalea obovatifolia Ortega

* Dalea obreniformis (Rydb.) Barneby

* Dalea polystachya (Sessé \& Moc.) Barneby

* Dalea prostrata Ortega

*Dalea reclinata (Cav.) Willd.

Dalea sericea Lag.

* Dalea thouinii Schrank

Dalea tomentosa (Cav.) Willd.

Dalea urceolata Greene

Dalea versicolor Zucc.

*Dalea zimapanica S. Schauer

* Desmanthus painteri (Britton \& Rose) Standl.

*Desmanthus pumilus (Schltdl.) J.F. Macbr.

Desmanthus virgatus (L.) Willd.

Desmodium affine Schltdl.

*Desmodium alamanii DC.

Desmodium amplifolium Hemsl.
Desmodium angustifolium (Kunth) DC.

Desmodium aparines (Link) DC.

Desmodium barbatum (L.) Benth.

*Desmodium bellum (S.F. Blake) B.G. Schub.

*Desmodium callilepis Hemsl.

Desmodium cinereum (Kunth) DC.

*Desmodium densiflorum Hemsl.

Desmodium distortum (Aubl.) J.F. Macbr.

*Desmodium ghiesbreghtii Hemsl.

Desmodium grahamii A. Gray

Desmodium hartwegianum Hemsl.

Desmodium infractum DC.

Desmodium intortum (Mill.) Urb.

*Desmodium jaliscanum S. Watson

Desmodium johnstonii Standl. ex B.G. Schub.

Desmodium lavanduliflorum Standl.

*Desmodium macropodium Hemsl.

*Desmodium macrostachyum Hemsl.

*Desmodium michelianum (Schindl.) B.G. Schub. \& McVaugh

*Desmodium michoacanum B.G. Schub. \& McVaugh

Desmodium molliculum (Kunth) DC.

Desmodium neomexicanum A. Gray

Desmodium nicaraguense Oerst.

Desmodium orbiculare Schltdl.

Desmodium plicatum Schltdl. \& Cham.

Desmodium prehensile Schltdl.

Desmodium pringlei S. Watson

Desmodium procumbens (Mill.) Hitchc.

Desmodium retinens Schltdl.

Desmodium scorpiurus (Sw.) Desv.

Desmodium sericophyllum Schltdl.

Desmodium skinneri Benth. ex Hemsl.

Desmodium strobilaceum Schltdl.

*Desmodium subsessile Schltdl.

*Desmodium sumichrastii (Schindl.) Standl.

Desmodium tortuosum (Sw.) DC.

Desmodium uncinatum (Jacq.) DC.

*Desmodium urarioides (S.F. Blake) B.G. Schub. \& McVaugh Desmodium venustum Steud.

Diphysa floribunda Peyr.

*Diphysa macrocarpa Standl.

* Diphysa minutifolia Rose

*Diphysa puberulenta Rydb.

*Diphysa racemosa Rose

*Diphysa suberosa S. Watson

*Diphysa villosa Rydb. 
Apéndice 4: Continuación.

Entadopsis polystachya (L.) Britton

Enterolobium cyclocarpum (Jacq.) Griseb.

Eriosema diffusum (Kunth) G. Don

Eriosema grandiflorum (Schltdl. \& Cham.) G. Don

*Eriosema longicalyx Grear

*Eriosema multiflorum B.L. Rob.

*Eriosema palmeri S. Watson

Eriosema pulchellum (Kunth) G. Don

*Erythrina americana Mill.

* Erythrina batolobium Barneby \& Krukoff

*Erythrina breviflora DC.

*Erythrina lanata Rose

*Erythrina leptorhiza Moc. \& Sessé ex DC.

Erythrina mexicana Krukoff

Eysenhardtia polystachya (Ortega) Sarg.

* Eysenhardtia punctata Pennell

Galactia acapulcensis Rose

* Galactia incana (Rose) Standl.

Galactia multiflora B.L. Rob. \& Greenm.

Galactia striata (Jacq.) Urb.

*Galactia viridiflora (Rose) Standl.

Gliricidia sepium (Jacq.) Kunth ex Walp.

Haematoxylum brasiletto $\mathrm{H}$. Karst.

Harpalyce formosa Moc. \& Sessé ex DC.

* Harpalyce sousae Arroyo

* Indigofera cuernavacana Rose

* Indigofera densiflora M. Martens \& Galeotti

Indigofera jamaicensis Spreng.

Indigofera miniata Ortega

* Indigofera platycarpa Rose

Indigofera suffruticosa Mill.

Indigofera thibaudiana DC.

*Inga eriocarpa Benth.

Inga inicuil Schltdl. \& Cham. ex G. Don

Inga schiedeana Steud.

Inga vera Willd.

Lathyrus parvifolius S. Watson

Leptospron adenanthum (G. Mey.) A. Delgado

Leucaena diversifolia (Schltdl.) Benth.

Leucaena esculenta (Moc. \& Sessé ex DC.) Benth.

Leucaena leucocephala (Lam.) de Wit

*Leucaena macrophylla Benth.

Libidibia coriaria (Jacq.) Schltdl.

*Lonchocarpus andrieuxii M. Sousa

*Lonchocarpus argyrotrichus Harms

*Lonchocarpus caudatus Pittier
*Lonchocarpus eriophyllus Benth.

*Lonchocarpus hintonii Sandwith

Lonchocarpus rugosus Benth.

Lotus repens (G. Don) Sessé \& Moc. ex Standl. \& Steyerm.

Lupinus aschenbornii S. Schauer

*Lupinus barkeri Lindl.

*Lupinus campestris Cham. \& Schltdl.

Lupinus elegans Kunth

*Lupinus exaltatus Zucc.

*Lupinus geophilus Rose

*Lupinus hintonii C.P. Sm.

*Lupinus leptophyllus Cham. \& Schltdl.

*Lupinus marschallianus Sweet

Lupinus mexicanus Cerv. ex Lag.

Lupinus montanus Kunth

*Lupinus simulans Rose

*Lupinus splendens Rose

*Lupinus stipulatus J. Agardh

*Lupinus uncinatus Schltdl.

*Lupinus vernicius Rose

*Lupinus versicolor Sweet

Lysiloma acapulcense (Kunth) Benth.

Lysiloma divaricatum (Jacq.) J.F. Macbr.

*Lysiloma tergeminum Benth.

Machaerium biovulatum Micheli

Machaerium isadelphum (E. Mey.) Amshoff

Macroptilium atropurpureum (Sessé \& Moc. ex DC.) Urb.

Macroptilium gibbosifolium (Ortega) A. Delgado

Marina diffusa (Moric.) Barneby

* Marina gracillima (S. Watson) Barneby

* Marina greenmaniana (Rose) Barneby

* Marina minutiflora (Rose) Barneby

* Marina nutans (Cav.) Barneby

* Marina procumbens (DC.) Barneby

Marina scopa Barneby

* Marina spiciformis (Rose) Barneby

* Marina stilligera Barneby

*Marina unifoliata (B.L. Rob. \& Greenm.) Barneby

* Mariosousa acatlensis (Benth.) Seigler \& Ebinger

Mariosousa coulteri (Benth.) Seigler \& Ebinger

*Microlobius foetidus (Jacq.) M. Sousa \& G. Andrade

Mimosa aculeaticarpa Ortega

* Mimosa adenantheroides (M. Martens \& Galeotti) Benth.

* Mimosa affinis B.L. Rob.

Mimosa albida Humb. \& Bonpl. ex Willd.

*Mimosa benthamii J.F. Macbr. 
Apéndice 4: Continuación.

* Mimosa caerulea Rose

* Mimosa depauperata Benth.

Mimosa diplotricha C. Wright ex Sauvalle

* Mimosa egregia Sandwith

* Mimosa galeottii Benth.

Mimosa guatemalensis (Hook. \& Arn.) Benth.

* Mimosa lacerata Rose

* Mimosa nanchititlana R. Grether \& Barneby

Mimosa pigra $\mathrm{L}$.

Mimosa polyantha Benth.

Mimosa pudica L.

* Mimosa rhodocarpa (Britton \& Rose) R. Grether

* Mimosa tejupilcana R. Grether \& Mart.-Bern.

*Minkelersia multiflora Rose

Myroxylon balsamum (L.) Harms

Nissolia fruticosa Jacq.

* Nissolia hintonii Sandwith

*Nissolia laxior (B.L. Rob.) Rose

*Nissolia microptera Poir.

* Nissolia pringlei Rose

Pachyrhizus erosus (L.) Urb.

*Painteria leptophylla (DC.) Britton \& Rose

Parkinsonia aculeata L.

Pediomelum pentaphyllum (L.) J.W. Grimes

Pediomelum rhombifolium (Torr. \& A. Gray) Rydb.

Phaseolus anisotrichos Schltdl.

Phaseolus coccineus L.

* Phaseolus hintonii A. Delgado

Phaseolus lunatus L.

Phaseolus maculatus Scheele

* Phaseolus microcarpus Mart.

Phaseolus pauciflorus Sessé \& Moc. ex G. Don

Phaseolus pedicellatus Benth.

* Phaseolus perplexus A. Delgado

*Phaseolus pluriflorus Maréchal, Mascherpa \& Stainier

* Phaseolus tenellus Piper

Phaseolus vulgaris L.

Piscidia carthagenensis Jacq.

*Piscidia grandifolia (Donn. Sm.) I.M. Johnst.

Piscidia piscipula (L.) Sarg.

Pithecellobium dulce (Roxb.) Benth.

*Platymiscium lasiocarpum Sandwith

Poeppigia procera C. Presl

Prosopis juliflora (Sw.) DC.

Prosopis laevigata (Humb. \& Bonpl. ex Willd.) M.C. Johnst.

*Pterocarpus orbiculatus DC.
*Ramirezella calcoma Ochot.-Booth \& A. Delgado

Ramirezella strobilophora (B.L. Rob.) Rose

Rhynchosia discolor M. Martens \& Galeotti

Rhynchosia edulis Griseb.

Rhynchosia minima (L.) DC.

Rhynchosia precatoria (Humb. \& Bonpl. ex Willd.) DC.

* Rhynchosia prostrata Brandegee

Rhynchosia pyramidalis (Lam.) Urb.

Schnella herrerae Britton \& Rose

Senegalia berlandieri (Benth.) Britton \& Rose

*Senegalia macilenta (Rose) Britton \& Rose

Senegalia picachensis (Brandegee) Britton \& Rose

Senegalia tenuifolia (L.) Britton \& Rose

* Senna argentea (Kunth) H.S. Irwin \& Barneby

Senna atomaria (L.) H.S. Irwin \& Barneby

*Senna foetidissima (G. Don) H.S. Irwin \& Barneby

Senna fruticosa (Mill.) H.S. Irwin \& Barneby

Senna hirsuta (L.) H.S. Irwin \& Barneby

Senna holwayana (Rose) H.S. Irwin \& Barneby

Senna multiglandulosa (Jacq.) H.S. Irwin \& Barneby

Senna obtusifolia (L.) H.S. Irwin \& Barneby

Senna occidentalis (L.) Link

Senna pallida (Vahl) H.S. Irwin \& Barneby

Senna pendula (Humb. \& Bonpl. ex Willd.) H.S. Irwin \& Barneby

Senna pilifera (Vogel) H.S. Irwin \& Barneby

Senna racemosa (Mill.) H.S. Irwin \& Barneby

Senna septemtrionalis (Viv.) H.S. Irwin \& Barneby

Senna skinneri (Benth.) H.S. Irwin \& Barneby

Senna uniflora (Mill.) H.S. Irwin \& Barneby

Sesbania herbacea (Mill.) McVaugh

Sigmoidotropis speciosa (Kunth) A. Delgado

*Sphinga acatlensis (Benth.) Barneby \& J.W. Grimes

Stenolobium brachycarpum Benth.

Stylosanthes humilis Kunth

Tephrosia cinerea (L.) Pers.

*Tephrosia conzattii (Rydb.) Standl.

*Tephrosia cuernavacana (Rose) J.F. Macbr.

*Tephrosia macrantha B.L. Rob. \& Greenm. ex Pringle

*Tephrosia mexicana C.E. Wood

Tephrosia nicaraguensis Oerst.

*Tephrosia pogonocalyx C.E. Wood

Tephrosia vicioides Schltdl.

Teramnus uncinatus (L.) Sw.

Trifolium amabile Kunth

Trifolium wormskioldii Lehm.

*Vachellia bilimekii (J.F. Macbr.) Seigler \& Ebinger 
Apéndice 4: Continuación.

Vachellia campechiana (Mill.) Seigler \& Ebinger

Vachellia farnesiana (L.) Wight \& Arn.

Vachellia macracantha (Humb. \& Bonpl. ex Willd.) Seigler \& Ebinger

Vachellia pennatula (Schltdl. \& Cham.) Seigler \& Ebinger

Vicia americana Muhl. ex Willd.

Vicia humilis Kunth

Vicia ludoviciana Nutt. ex Torr. \& A. Gray

Vicia pulchella Kunth

Vigna luteola (Jacq.) Benth.

Zapoteca formosa (Kunth) H.M. Hern.

Zapoteca portoricensis (Jacq.) H.M. Hern.

Zapoteca tetragona (Willd.) H.M. Hern.

Zornia diphylla (L.) Pers.

Zornia reticulata $\mathrm{Sm}$.

Zornia thymifolia Kunth

\section{Fagaceae}

Quercus acutifolia Née

*Quercus affinis Scheidw.

*Quercus aristata Hook. \& Arn.

Quercus candicans Née

Quercus castanea Née

Quercus crassifolia Bonpl.

*Quercus crassipes Bonpl.

*Quercus deserticola Trel.

*Quercus diversifolia Née

*Quercus eduardi Trel.

Quercus elliptica Née

*Quercus frutex Trel.

Quercus glabrescens Benth.

*Quercus glaucescens Bonpl.

Quercus glaucoides M. Martens \& Galeotti

*Quercus greggii (A. DC.) Trel.

*Quercus hintonii E.F. Warb.

Quercus insignis M. Martens \& Galeotti

*Quercus laeta Liebm.

Quercus laurina Bonpl.

*Quercus liebmannii Oerst. ex Trel.

Quercus magnoliifolia Née

*Quercus martinezii C.H. Mull.

*Quercus mexicana Bonpl.

*Quercus microphylla Née

*Quercus obtusata Bonpl.

Quercus peduncularis Née

*Quercus planipocula Trel.
Quercus polymorpha Schltdl. \& Cham.

*Quercus potosina Trel.

*Quercus repanda Bonpl.

*Quercus resinosa Liebm.

Quercus rugosa Née

Quercus salicifolia Née

**Quercus sanchez-colinii Martínez

*Quercus sartorii Liebm.

*Quercus scytophylla Liebm.

*Quercus splendens Née

*Quercus subspathulata Trel.

*Quercus urbanii Trel.

*Quercus $\times$ dysophylla Benth.

\section{Fouquieriaceae}

*Fouquieria formosa Kunth

\section{Garryaceae}

Garrya laurifolia Hartw. ex Benth.

* Garrya longifolia Rose

\section{Gentianaceae}

* Gentiana bicuspidata (G. Don) Briq.

* Gentiana caliculata Lex.

Gentiana ovatiloba Kusn.

* Gentiana perpusilla Brandegee

* Gentiana spathacea Kunth

Gentianella amarella (L.) Börner

Gyrandra brachycalyx (Standl. \& L.O. Williams) G. Mans.

Halenia brevicornis (Kunth) G. Don

Halenia crassiuscula B.L. Rob. \& Seaton

*Halenia plantaginea (Kunth) G. Don

Zeltnera quitensis (Kunth) G. Mans.

\section{Geraniaceae}

*Geranium bellum Rose

*Geranium cruceroense R. Knuth

* Geranium deltoideum Rydb.

*Geranium hernandesii DC.

*Geranium latum Small

*Geranium lilacinum R. Knuth

*Geranium lozanoi Rose

*Geranium mexicanum Kunth

*Geranium potentillifolium DC.

*Geranium schiedeanum Schltdl.

Geranium seemannii Peyr. 
Apéndice 4: Continuación.

\section{Gesneriaceae}

Achimenes antirrhina (DC.) C.V. Morton

Achimenes erecta (Lam.) H.P. Fuchs

*Achimenes flava C.V. Morton

Achimenes grandiflora (Schiede) DC.

Achimenes heterophylla (Mart.) DC.

*Achimenes obscura C.V. Morton

*Achimenes patens Benth.

Achimenes pedunculata Benth.

\section{Grossulariaceae}

*Ribes affine Kunth

Ribes ciliatum Humb. \& Bonpl. ex Roem. \& Schult.

Ribes microphyllum Kunth

\section{Haloragaceae}

Myriophyllum heterophyllum Michx.

Myriophyllum hippuroides Nutt. ex Torr. \& A. Gray

Myriophyllum quitense Kunth

\section{Hamamelidaceae}

Matudaea trinervia Lundell

\section{Hydrangeaceae}

Philadelphus mexicanus Schltdl.

\section{Hydroleaceae}

Hydrolea spinosa $\mathrm{L}$.

\section{Hypericaceae}

Hypericum formosum Kunth

Hypericum pauciflorum Kunth

Hypericum philonotis Schltdl. \& Cham.

Hypericum silenoides Juss.

\section{Juglandaceae}

*Juglans pyriformis Liebm.

\section{Krameriaceae}

Krameria revoluta $\mathrm{O}$. Berg

Krameria secundiflora Moc. \& Sessé ex DC.

\section{Lamiaceae}

Agastache mexicana (Kunth) Lint \& Epling Asterohyptis mociniana (Benth.) Epling

*Asterohyptis stellulata (Benth.) Epling
Cantinoa americana (Aubl.) Harley \& J.F.B. Pastore

Cantinoa mutabilis (Rich.) Harley \& J.F.B. Pastore

*Clinopodium macrostemum (Moc. \& Sessé ex Benth.) Kuntze

*Condea albida (Kunth) Harley \& J.F.B. Pastore

Condea subtilis (Epling) Harley \& J.F.B. Pastore

*Cunila lythrifolia Benth.

* Cunila pycnantha B.L. Rob. \& Greenm.

Hedeoma costata A. Gray

Hedeoma piperita Benth.

Hyptis capitata Jacq.

Lepechinia caulescens (Ortega) Epling

*Lepechinia nelsonii (Fernald) Epling

Lepechinia schiedeana (Schltdl.) Vatke

Mentha canadensis L.

Mesosphaerum pectinatum (L.) Kuntze

* Mesosphaerum septentrionale (Epling) Harley \& J.F.B. Pastore

Mesosphaerum suaveolens (L.) Kuntze

Mesosphaerum urticoides (Kunth) Kuntze

*Salvia albocaerulea Linden

Salvia carnea Kunth

* Salvia chamaedryoides Cav.

Salvia cinnabarina M. Martens \& Galeotti

* Salvia clinopodioides Kunth

Salvia coccinea Buc'hoz ex Etl.

*Salvia concolor Lamb. ex Benth.

* Salvia dichlamys Epling

* Salvia dryophila Epling

*Salvia elegans Vahl

Salvia excelsa Benth.

* Salvia filifolia Ramamoorthy

* Salvia fluviatilis Fernald

* Salvia fulgens Cav.

* Salvia gesneriflora Lindl. \& Paxton

*Salvia glechomifolia Kunth

* Salvia guadalajarensis Briq.

* Salvia helianthemifolia Benth.

* Salvia hirsuta Jacq.

Salvia hispanica L.

*Salvia iodantha Fernald

* Salvia keerlii Benth.

* Salvia laevis Benth.

Salvia lasiantha Benth.

Salvia lasiocephala Hook. \& Arn.

Salvia lavanduloides Kunth

* Salvia leptostachys Benth.

Salvia leucantha Cav. 
Apéndice 4: Continuación.

*Salvia longispicata M. Martens \& Galeotti

* Salvia longistyla Benth.

* Salvia melissodora Lag.

*Salvia mexicana L.

Salvia microphylla Kunth

Salvia misella Kunth

Salvia mocinoi Benth.

* Salvia moniliformis Fernald

* Salvia nepetoides Kunth

* Salvia oreopola Fernald

* Salvia patens Cav.

Salvia polystachia Cav.

* Salvia prunelloides Kunth

Salvia pulchella DC.

Salvia purpurea Cav.

Salvia reflexa Hornem.

*Salvia remota Benth.

Salvia reptans Jacq.

* Salvia rhyacophila (Fernald) Epling

**Salvia rzedowskii Ramamoorthy

* Salvia sessei Benth.

* Salvia setulosa Fernald

* Salvia stricta Sessé \& Moc.

Salvia tiliifolia Vahl

* Salvia xalapensis Benth.

Scutellaria dumetorum Schltdl.

* Scutellaria hintoniana Epling

Stachys agraria Schltdl. \& Cham.

* Stachys boraginoides Cham. \& Schltdl.

Stachys coccinea Ortega

Stachys eriantha Benth.

* Stachys globosa Epling

* Stachys keerlii Benth.

*Stachys nepetifolia Desf.

* Stachys parvifolia M. Martens \& Galeotti

Stachys pilosissima M. Martens \& Galeotti

Stachys radicans Epling

*Stachys rotundifolia Moc. \& Sessé ex Benth.

*Stachys sanchezii Rzed. \& A. García

*Vitex mollis Kunth

*Vitex pyramidata B.L. Rob.

\section{Lentibulariaceae}

*Pinguicula acuminata Benth.

* Pinguicula caudata Schltdl.

Pinguicula crenatiloba A. DC.
*Pinguicula macrophylla Kunth

Pinguicula moranensis Kunth

*Pinguicula oblongiloba DC.

*Pinguicula parvifolia B.L. Rob.

Utricularia amethystina Salzm. ex A. St.-Hil. \& Girard

Utricularia gibba L.

*Utricularia hintonii P. Taylor

Utricularia livida E. Mey.

Utricularia macrorhiza Leconte

*Utricularia perversa P. Taylor

Utricularia subulata L.

\section{Linaceae}

Linum australe A. Heller

*Linum mexicanum Kunth

*Linum orizabae Planch.

**Linum rzedowskii Arreguín

Linum schiedeanum Schltdl. \& Cham.

\section{Loasaceae}

Eucnide hirta (G. Don) H.J. Thomps. \& W.R. Ernst

* Gronovia longiflora Rose

Gronovia scandens L.

Klaprothia fasciculata (C. Presl) Poston

Mentzelia aspera L.

Mentzelia hispida Willd.

\section{Loganiaceae}

Mitreola petiolata (J.F. Gmel.) Torr. \& A. Gray

\section{Loranthaceae}

*Cladocolea andrieuxii Tiegh.

* Cladocolea diversifolia (Benth.) Kuijt

*Cladocolea grahamii (Benth.) Tiegh.

*Cladocolea loniceroides (Tiegh.) Kuijt

*Cladocolea microphylla (Kunth) Kuijt

*Cladocolea pedicellata Kuijt

Psittacanthus calyculatus (DC.) G. Don

*Psittacanthus palmeri (S. Watson) Barlow \& Wiens

Psittacanthus schiedeanus (Schltdl. \& Cham.) G. Don

Struthanthus brachybotrys Standl. \& Steyerm.

*Struthanthus grahamii (Benth.) Standl.

Struthanthus interruptus (Kunth) G. Don

\section{Lythraceae}

Cuphea aequipetala Cav. 
Apéndice 4: Continuación.

Cuphea angustifolia Jacq. ex Koehne

* Cuphea avigera B.L. Rob. \& Seaton

* Cuphea bustamanta Lex.

Cuphea cyanea DC.

*Cuphea heterophylla Benth.

Cuphea hookeriana Walp.

Cuphea hyssopifolia Kunth

*Cuphea jorullensis Kunth

* Cuphea koehneana Rose

*Cuphea lanceolata W.T. Aiton

Cuphea leptopoda Hemsl.

Cuphea llavea Lex.

*Cuphea lobophora Koehne

Cuphea lophostoma Koehne

*Cuphea micropetala Kunth

* Cuphea paucipetala S.A. Graham

*Cuphea procumbens Ortega

*Cuphea salicifolia Cham. \& Schltdl.

* Cuphea schumannii Koehne

Cuphea secundiflora Moc. \& Sessé ex DC.

Cuphea tolucana Peyr.

Cuphea wrightii A. Gray

Heimia salicifolia Link

Lythrum alatum Pursh

* Lythrum album Kunth

Lythrum gracile Benth.

Lythrum vulneraria Aiton ex Schrank

Rotala ramosior (L.) Koehne

\section{Malpighiaceae}

Bronwenia cornifolia (Kunth) W.R. Anderson \& C. Davis

Bunchosia biocellata Schltdl.

Bunchosia lindeniana A. Juss.

*Bunchosia palmeri S. Watson

Byrsonima bucidifolia Standl.

Byrsonima crassifolia (L.) Kunth

* Calcicola parvifolia (A. Juss.) W.R. Anderson \& C. Davis

* Callaeum coactum D.M. Johnson

Callaeum macropterum (DC.) D.M. Johnson

*Echinopterys eglandulosa (A. Juss.) Small

Galphimia glauca Cav.

* Gaudichaudia cycloptera (DC.) W.R. Anderson

* Gaudichaudia cynanchoides Kunth

Gaudichaudia hirtella (Rich.) S.L. Jessup

Gaudichaudia mucronata (Moc. \& Sessé ex DC.) A. Juss.

Heteropterys brachiata (L.) DC.
Heteropterys laurifolia (L.) A. Juss.

Malpighia glabra L.

* Malpighia hintonii Bullock

* Malpighia mexicana A. Juss.

*Mascagnia polybotrya (A. Juss.) Nied.

* Psychopterys dipholiphylla (Small) W.R. Anderson \& S. Corso

*Tetrapterys mexicana Hook. \& Arn.

\author{
Malvaceae \\ Abutilon abutiloides (Jacq.) Garcke ex Hochr. \\ Abutilon divaricatum Turcz. \\ *Abutilon dugesii S. Watson \\ Abutilon reventum $\mathrm{S}$. Watson \\ *Abutilon simulans Rose \\ *Allowissadula sessei (Lag.) D.M. Bates \\ Anoda acerifolia Cav. \\ Anoda crenatiflora Ortega \\ Anoda cristata (L.) Schltdl. \\ *Anoda hintoniorum Fryxell \\ *Anoda paniculata Hochr. \\ Anoda pentaschista A. Gray \\ *Anoda pubescens Schltdl. \\ Anoda thurberi A. Gray \\ *Ayenia abutilifolia (Turcz.) Turcz. \\ * Ayenia berlandieri S. Watson \\ *Ayenia jaliscana $\mathrm{S}$. Watson \\ * Ayenia mexicana Turcz. \\ Ayenia micrantha Standl. \\ Ayenia purpusii Brandegee \\ Ayenia pusilla L. \\ Byttneria aculeata (Jacq.) Jacq. \\ *Byttneria atrata Bullock \\ *Ceiba acuminata (S. Watson) Rose \\ Ceiba aesculifolia (Kunth) Britten \& Baker f. \\ Ceiba pentandra (L.) Gaertn. \\ Chiranthodendron pentadactylon Larreat. \\ Fuertesimalva jacens (S. Watson) Fryxell \\ Fuertesimalva limensis (L.) Fryxell \\ Guazuma ulmifolia Lam. \\ Helicteres guazumifolia Kunth \\ Heliocarpus americanus L. \\ Heliocarpus occidentalis Rose \\ *Heliocarpus pallidus Rose \\ Heliocarpus terebinthinaceus (DC.) Hochr. \\ *Heliocarpus velutinus Rose \\ Herissantia crispa (L.) Brizicky
}


Apéndice 4: Continuación.

Hermannia inflata Link \& Otto

Hibiscus phoeniceus Jacq.

Kearnemalvastrum lacteum (Aiton) D.M. Bates

Kearnemalvastrum subtriflorum (Lag.) D.M. Bates

*Kosteletzkya blanchardii Fryxell

Malachra fasciata Jacq.

Malvastrum americanum (L.) Torr.

Malvastrum bicuspidatum (S. Watson) Rose

Malvastrum coromandelianum (L.) Garcke

Malvaviscus arboreus Cav.

Malvella leprosa (Ortega) Krapov.

*Melochia corymbosa (C. Presl) Meisn. ex Steud.

Melochia nodiflora Sw.

Melochia pyramidata L.

Melochia tomentosa $\mathrm{L}$.

Melochia urticifolia (Turcz.) Standl.

Neobrittonia acerifolia (G. Don) Hochr.

* Pavonia candida (DC.) Fryxell

* Pavonia chlorantha (Kunth) Fryxell

*Pavonia oxyphylla (DC.) Fryxell

*Pavonia pulidoae Fryxell

Periptera punicea (Lag.) DC.

Phymosia rosea (DC.) Kearney

*Phymosia rzedowskii Fryxell

* Physodium adenodes (Goldberg) Fryxell

Pseudabutilon ellipticum (Schltdl.) Fryxell

Pseudobombax ellipticum (Kunth) Dugand

*Robinsonella hintonii Fryxell

Sida abutifolia Mill.

Sida acuta Burm. f.

Sida barclayi Baker f.

Sida ciliaris L.

Sida elliottii Torr. \& A. Gray

Sida glabra Mill.

Sida haenkeana C. Presl

* Sida linearis Cav.

* Sida monticola Fryxell

* Sida prolifica Fryxell \& S.D. Koch

Sida rhombifolia L.

Sphaeralcea angustifolia (Cav.) G. Don

Tarasa antofagastana (Phil.) Krapov.

*Tarasa geranioides (Schltdl. \& Cham.) Krapov.

Tilia americana $\mathrm{L}$.

*Trichospermum galeottii (Turcz.) Kosterm.

Triumfetta bogotensis DC.

*Triumfetta columnaris Hochr.
*Triumfetta coriacea Hochr.

*Triumfetta galeottiana Turcz.

*Triumfetta goldmanii Rose

*Triumfetta heliocarpoides Bullock

Triumfetta lappula L.

Triumfetta polyandra Sessé \& Moc. ex DC.

*Triumfetta simplicifolia (Sessé \& Moc.) Fryxell

*Triumfetta stellata Lay

*Waltheria fryxellii J.G. Saunders

Waltheria indica $\mathrm{L}$.

*Waltheria pringlei Rose \& Standl.

Wissadula amplissima (L.) R.E. Fr.

\section{Martyniaceae}

Martynia annua L.

Proboscidea louisiana (Mill.) Thell.

Proboscidea triloba (Schltdl. \& Cham.) Decne.

\section{Melastomataceae}

Arthrostemma alatum Triana

Clidemia matudae L.O. Williams

Conostegia xalapensis (Bonpl.) D. Don ex DC.

*Heterocentron mexicanum Hook. \& Arn.

Heterocentron subtriplinervium (Link \& Otto) A. Braun \& C.D.

Bouché

Leandra cornoides (Schltdl. \& Cham.) Cogn.

Miconia glaberrima (Schltdl.) Naudin

*Monochaetum calcaratum (DC.) Triana

Monochaetum floribundum (Schltdl.) Naudin

*Tibouchina galeottiana (Naudin) Cogn.

Tibouchina longifolia (Vahl) Baill.

*Tibouchina scabriuscula (Schltdl.) Cogn.

*Tibouchina tortuosa (Bonpl.) Almeda

\section{Meliaceae}

Cedrela odorata L.

Guarea glabra Vahl

Swietenia humilis Zucc.

Trichilia americana (Sessé \& Moc.) T.D. Penn.

Trichilia havanensis Jacq.

Trichilia hirta L.

\section{Menispermaceae}

Cissampelos pareira $\mathrm{L}$.

Cocculus diversifolius DC.

*Disciphania mexicana Bullock 
Apéndice 4: Continuación.

\section{Menyanthaceae \\ Nymphoides fallax Ornduff}

\section{Molluginaceae}

Mollugo verticillata $\mathrm{L}$.

\section{Montiaceae}

Calandrinia acaulis Kunth

Calandrinia ciliata (Ruiz \& Pav.) DC.

Claytonia perfoliata Donn ex Willd.

Montia chamissoi (Ledeb. ex Spreng.) Greene

Montia fontana L.

*Phemeranthus napiformis (DC.) G. Ocampo

\section{Moraceae}

Brosimum alicastrum Sw.

Castilla elastica Sessé ex Cerv.

Dorstenia contrajerva L.

Dorstenia drakena $\mathrm{L}$.

Ficus aurea Nutt.

Ficus citrifolia Mill.

Ficus cotinifolia Kunth

Ficus crocata (Miq.) Miq.

Ficus insipida Willd.

*Ficus lapathifolia (Liebm.) Miq.

Ficus maxima Mill.

Ficus obtusifolia Kunth

Ficus pertusa L. f.

*Ficus petiolaris Kunth

Ficus velutina Humb. \& Bonpl. ex Willd.

Morus celtidifolia Kunth

Trophis racemosa (L.) Urb.

\section{Muntingiaceae \\ Muntingia calabura L.}

\section{Myricaceae}

Morella cerifera (L.) Small

\section{Myrtaceae}

Eugenia acapulcensis Steud.

Eugenia capuli (Schltdl. \& Cham.) Hook. \& Arn.

Myrcianthes fragrans (Sw.) McVaugh

Psidium guajava L.

Psidium sartorianum (O. Berg) Nied.

\section{Nyctaginaceae}

Allionia choisyi Standl.

Allionia incarnata $\mathrm{L}$.

Boerhavia coccinea Mill.

Boerhavia erecta $\mathrm{L}$.

Commicarpus scandens (L.) Standl.

Mirabilis aggregata (Ortega) Cav.

Mirabilis albida (Walter) Heimerl

Mirabilis glabrifolia (Ortega) I.M. Johnst.

Mirabilis jalapa $\mathrm{L}$.

Mirabilis longiflora L.

Mirabilis melanotricha (Stand1.) Spellenb.

*Mirabilis pringlei Weath.

* Mirabilis sanguinea Heimerl

Mirabilis violacea (L.) Heimerl

Mirabilis viscosa Cav.

Okenia hypogaea Schltdl. \& Cham.

Pisonia aculeata L.

Pisoniella arborescens (Lag. \& Rodr.) Standl.

*Salpianthus arenarius Bonpl.

\section{Oleaceae}

Cartrema americana (L.) G.L. Nesom

Forestiera phillyreoides (Benth.) Torr.

Forestiera reticulata Torr.

Fraxinus purpusii Brandegee

Fraxinus uhdei (Wenz.) Lingelsh.

*Menodora helianthemoides Bonpl.

\section{Onagraceae}

Epilobium ciliatum Raf.

*Epilobium denticulatum Ruiz \& Pav.

Fuchsia arborescens Sims

*Fuchsia decidua Standl.

Fuchsia encliandra Steud.

*Fuchsia fulgens DC.

Fuchsia lycioides Andrews

Fuchsia microphylla Kunth

*Fuchsia obconica Breedlove

Fuchsia thymifolia Kunth

Gaura drummondii (Spach) Torr. \& A. Gray

* Gaura mutabilis Cav.

Gongylocarpus rubricaulis Schltdl. \& Cham.

Lopezia miniata Lag. ex DC.

Lopezia racemosa Cav.

*Lopezia trichota Schltdl. 
Apéndice 4: Continuación.

* Lopezia violacea Rose

Ludwigia clavellina M. Gómez

Ludwigia erecta (L.) H. Hara

Ludwigia octovalvis (Jacq.) P.H. Raven

Ludwigia palustris (L.) Elliott

Ludwigia peploides (Kunth) P.H. Raven

* Oenothera deserticola (Loes.) Munz

Oenothera elata Kunth

Oenothera epilobiifolia Kunth

Oenothera flava (A. Nelson) Garrett

Oenothera hexandra (Ortega) W.L. Wagner \& Hoch

Oenothera kunthiana (Spach) Munz

Oenothera laciniata Hill

Oenothera pubescens Willd. ex Spreng.

Oenothera rosea L'Hér. ex Aiton

Oenothera suffrutescens (Ser.) W.L. Wagner \& Hoch

Oenothera tetraptera Cav.

* Oenothera tubifera Ser.

\section{Opiliaceae}

Agonandra racemosa (DC.) Standl.

\section{Orobanchaceae}

Agalinis peduncularis (Benth.) Pennell

Buchnera obliqua Benth.

Buchnera pusilla Kunth

Castilleja arvensis Schltdl. \& Cham.

*Castilleja aurea B.L. Rob. \& Greenm.

*Castilleja gracilis Benth.

* Castilleja lithospermoides Kunth

* Castilleja macrostigma B.L. Rob.

* Castilleja moranensis Kunth

Castilleja pectinata M. Martens \& Galeotti

*Castilleja scorzonerifolia Kunth

*Castilleja stipifolia G.L. Nesom

Castilleja tenuiflora Benth.

*Castilleja tenuifolia M. Martens \& Galeotti

*Castilleja tolucensis Kunth

Conopholis alpina Liebm.

Escobedia laevis Schltdl. \& Cham.

* Lamourouxia brachyantha Greenm.

*Lamourouxia dasyantha (Cham. \& Schltdl.) W.R. Ernst

Lamourouxia multifida Kunth

*Lamourouxia nelsonii B.L. Rob. \& Greenm.

*Lamourouxia rhinanthifolia Kunth

*Lamourouxia tenuifolia M. Martens \& Galeotti
Lamourouxia viscosa Kunth

Lamourouxia xalapensis Kunth

Melasma physalodes (D. Don) Melch.

* Orobanche dugesii (S. Watson) Munz

Orobanche ludoviciana Nutt.

Pedicularis canadensis L.

*Pedicularis mexicana Zucc. ex Benth.

Pedicularis orizabae Schltdl. \& Cham.

* Seymeria decurva Benth.

* Silviella prostrata (Kunth) Pennell

*Silviella serpyllifolia (Kunth) Pennell

\section{Oxalidaceae}

Oxalis alpina (Rose) Rose ex R. Knuth

Oxalis corniculata L.

Oxalis decaphylla Kunth

Oxalis dimidiata Donn. Sm.

Oxalis divergens Benth. ex Lindl.

Oxalis frutescens L.

* Oxalis gregaria (Rose) R. Knuth

* Oxalis hernandezii DC.

Oxalis jacquiniana Kunth

Oxalis latifolia Kunth

Oxalis lunulata Zucc.

Oxalis nelsonii (Small) R. Knuth

Oxalis tetraphylla Cav.

\section{Papaveraceae}

Argemone mexicana L.

Argemone ochroleuca Sweet

Argemone platyceras Link \& Otto

Bocconia arborea $\mathrm{S}$. Watson

Bocconia frutescens L.

Eschscholzia californica Cham.

\section{Passifloraceae}

Passiflora adenopoda DC.

Passiflora biflora Lam.

Passiflora bryonioides Kunth

* Passiflora colimensis Mast. \& Rose

Passiflora coriacea Juss.

*Passiflora exsudans Zucc.

Passiflora filipes Benth.

Passiflora foetida $\mathrm{L}$.

Passiflora jorullensis Kunth

Passiflora ligularis Juss. 
Apéndice 4: Continuación.

Passiflora mexicana Juss.

Passiflora pavonis Mast.

Passiflora porphyretica Mast.

Passiflora sicyoides Schltdl. \& Cham.

Passiflora suberosa L.

Passiflora subpeltata Ortega

*Turnera callosa Urb.

Turnera ulmifolia L.

\section{Pentaphylacaceae}

*Cleyera integrifolia (Benth.) Choisy

Ternstroemia lineata DC.

Ternstroemia sylvatica Schltdl. \& Cham.

Ternstroemia tepezapote Schltdl. \& Cham.

\section{Petiveriaceae}

Petiveria alliacea $\mathrm{L}$.

Rivina humilis L.

Trichostigma octandrum (L.) H. Walter

\section{Phrymaceae}

Erythranthe glabrata (Kunth) G.L. Nesom

Mimulus cardinalis Douglas ex Benth.

\section{Phyllanthaceae}

Phyllanthus caroliniensis Walter

*Phyllanthus galeottianus Baill.

Phyllanthus mocinianus Baill.

\section{Phyllonomaceae}

Phyllonoma laticuspis (Turcz.) Engl.

\section{Phytolaccaceae}

Phytolacca icosandra L.

Phytolacca rivinoides Kunth \& C.D. Bouché

Phytolacca rugosa A. Braun \& C.D. Bouché

\section{Picramniaceae}

Alvaradoa amorphoides Liebm.

Picramnia antidesma Sw.

\section{Plantaginaceae}

Bacopa monnieri (L.) Wettst.

Callitriche deflexa A. Braun ex Hegelm.

Callitriche heterophylla Pursh

Linaria canadensis (L.) Dum. Cours.
Maurandya antirrhiniflora Humb. \& Bonpl. ex Willd.

Maurandya barclayana Lindl.

Maurandya erubescens (D. Don) A. Gray

Mecardonia procumbens (Mill.) Small

Nuttallanthus canadensis (L.) D.A. Sutton

Penstemon barbatus (Cav.) Roth

Penstemon campanulatus (Cav.) Willd.

Penstemon gentianoides (Kunth) Poir.

*Penstemon hartwegii Benth.

*Penstemon miniatus Lindl.

*Penstemon roseus (Sweet) G. Don

* Plantago alismatifolia Pilg.

Plantago australis Lam.

Plantago linearis Kunth

Plantago nivea Kunth

* Plantago tolucensis Pilg.

Plantago tubulosa Decne.

Russelia coccinea (L.) Wettst.

*Russelia cuneata B.L. Rob.

* Russelia floribunda Kunth

*Russelia jaliscensis B.L. Rob.

Russelia parvifolia Carlson

*Russelia retrorsa Greene

Russelia sarmentosa Jacq.

*Russelia tenuis Lundell

*Russelia ternifolia Kunth

Russelia verticillata Kunth

*Russelia villosa Lundell

Schistophragma pusillum Benth.

Sibthorpia rotundifolia (Ruiz \& Pav.) Edwin

Stemodia macrantha B.L. Rob.

Veronica americana Schwein. ex Benth.

Veronica peregrina $\mathrm{L}$.

Plumbaginaceae

*Plumbago pulchella Boiss.

\section{Podostemaceae}

Tristicha trifaria (Bory ex Willd.) Spreng.

\section{Polemoniaceae}

Bonplandia geminiflora Cav.

Cobaea scandens Cav.

*Ipomopsis pinnata (Cav.) V.E. Grant

Loeselia coerulea (Cav.) G. Don

Loeselia glandulosa (Cav.) G. Don 
Apéndice 4: Continuación.

Loeselia mexicana (Lam.) Brand

*Polemonium grandiflorum Benth.

*Polemonium mexicanum Cerv. ex Lag.

\section{Polygalaceae}

*Monnina ciliolata DC.

Monnina xalapensis Kunth

Polygala alba Nutt.

*Polygala appressipilis S.F. Blake

* Polygala brachytropis S.F. Blake

*Polygala compacta Rose

Polygala glochidiata Kunth

Polygala gracillima S. Watson

* Polygala scoparia Kunth

*Polygala subalata S. Watson

Polygala tenella Willd.

\section{Polygonaceae}

Antigonon leptopus Hook. \& Arn.

Eriogonum jamesii Benth.

Persicaria amphibia (L.) Gray

Persicaria hydropiperoides (Michx.) Small

Persicaria punctata (Elliott) Small

Polygonum acuminatum Kunth

*Rumex flexicaulis Rech. f.

Rumex mexicanus Meisn.

* Ruprechtia fusca Fernald

\section{Portulacaceae}

Portulaca mexicana P. Wilson

Portulaca oleracea L.

Portulaca pilosa L.

\section{Primulaceae}

Ardisia compressa Kunth

Ardisia escallonioides Schltdl. \& Cham.

Ardisia revoluta Kunth

Myrsine coriacea (Sw.) R. Br. ex Roem. \& Schult.

Myrsine juergensenii (Mez) Ricketson \& Pipoly

Parathesis melanosticta (Schltdl.) Hemsl.

* Parathesis villosa Lundell

Synardisia venosa (Mast.) Lundell

\section{Ranunculaceae}

Clematis dioica $\mathrm{L}$.

Clematis grossa Benth.
* Clematis rhodocarpa Rose

*Delphinium bicornutum Hemsl.

*Delphinium pedatisectum Hemsl.

Ranunculus aquatilis L.

Ranunculus cymbalaria Pursh

Ranunculus dichotomus Moc. \& Sessé ex DC.

Ranunculus donianus Pritz. ex Walp.

Ranunculus fasciculatus Sessé \& Moc.

Ranunculus hydrocharoides A. Gray

Ranunculus macranthus Scheele

Ranunculus multicaulis D. Don

Ranunculus peruvianus Pers.

Ranunculus petiolaris Kunth ex DC.

Ranunculus praemorsus Kunth ex DC.

Ranunculus sierrae-orientalis (L.D. Benson) G.L. Nesom

*Thalictrum cuernavacanum Rose

*Thalictrum gibbosum Lecoy.

*Thalictrum hernandezii Tausch

*Thalictrum pachucense Rose

*Thalictrum peltatum DC.

*Thalictrum pubigerum Benth.

*Thalictrum strigillosum Hemsl.

\section{Rhamnaceae}

Adolphia infesta (Kunth) Meisn.

*Ceanothus buxifolius Willd. ex Schult. f.

Ceanothus caeruleus Lag.

Colubrina celtidifolia (Schltdl. \& Cham.) Schltdl.

Colubrina elliptica (Sw.) Brizicky \& W.L. Stern

Colubrina greggii S. Watson

*Colubrina macrocarpa (Cav.) G. Don

Colubrina triflora Brongn. ex G. Don

Condalia mexicana Schltdl.

*Condalia velutina I.M. Johnst.

*Frangula hintonii (M.C. Johnst. \& L.A. Johnst.) A. Pool

*Frangula microphylla (Humb. \& Bonpl. ex Schult.) Grubov

Frangula mucronata (Schltdl.) Grubov

*Frangula palmeri (S. Watson) Grubov

* Gouania conzattii Greenm.

Gouania lupuloides (L.) Urb.

Karwinskia humboldtiana (Schult.) Zucc.

*Karwinskia mollis Schltdl.

* Karwinskia venturae R. Fernández Krugiodendron ferreum (Vahl) Urb.

Rhamnus serrata Humb. \& Bonpl. ex Willd.

Sageretia elegans (Kunth) Brongn. 
Apéndice 4: Continuación.

\section{Rosaceae}

Acaena elongata L.

* Cercocarpus fothergilloides Kunth

*Cercocarpus macrophyllus C.K. Schneid.

Crataegus mexicana DC.

*Fragaria mexicana Schltdl.

Geum aleppicum Jacq.

Holodiscus fissus (Lindl.) C.K. Schneid.

*Holodiscus pachydiscus (Rydb.) Standl.

Lachemilla aphanoides (Mutis ex L. f.) Rothm.

**Lachemilla mexiquense D.F. Morales-B.

Lachemilla orbiculata (Ruiz \& Pav.) Rydb.

Lachemilla pinnata (Ruiz \& Pav.) Rothm.

Lachemilla procumbens (Rose) Rydb.

Lachemilla sibbaldiifolia (Kunth) Rydb.

*Lachemilla velutina (S. Watson) Rydb.

Lachemilla vulcanica (Schltdl. \& Cham.) Rydb.

Malacomeles denticulata (Kunth) G.N. Jones

Photinia microcarpa Standl.

* Potentilla candicans Humb. \& Bonpl. ex Nestl.

Potentilla haematochrous Lehm.

* Potentilla ranunculoides Humb. \& Bonpl. ex Nestl.

* Potentilla richardii Lehm.

Potentilla rivalis Nutt.

*Potentilla rubra Willd. ex D.F.K. Schltdl.

Potentilla staminea Rydb.

Prunus brachybotrya Zucc.

* Prunus ferruginea (Ser.) Steud.

*Prunus microphylla (Kunth) Hemsl.

Prunus serotina Ehrh.

Rubus adenotrichos Schltdl.

* Rubus caudatisepalus Calderón

* Rubus cymosus Rydb.

*Rubus liebmannii Focke

Rubus pringlei Rydb.

* Rubus pumilus Focke

*Rubus uhdeanus Focke

Sibbaldia procumbens L.

\section{Rubiaceae}

Arachnothryx buddleioides (Benth.) Planch.

Arachnothryx jurgensenii (Hemsl.) Borhidi

*Arachnothryx leucophylla (Kunth) Planch.

Augusta rivalis (Benth.) J.H. Kirkbr.

Borreria remota (Lam.) Bacigalupo \& E.L. Cabral

Borreria suaveolens G. Mey.
Borreria verticillata (L.) G. Mey.

* Bouvardia capitata Bullock

*Bouvardia chrysantha Mart.

*Bouvardia cordifolia DC.

Bouvardia laevis M. Martens \& Galeotti

*Bouvardia loeseneriana Standl.

*Bouvardia longiflora (Cav.) Kunth

Bouvardia multiflora (Cav.) Schult. \& Schult. f.

*Bouvardia obovata Kunth

*Bouvardia standleyana W.H. Blackw.

Bouvardia ternifolia (Cav.) Schltdl.

Cephalanthus salicifolius Bonpl.

Chiococca alba (L.) Hitchc.

Chiococca pachyphylla Wernham

Crusea calocephala DC.

Crusea coccinea DC.

Crusea diversifolia (Kunth) W.R. Anderson

Crusea hispida (Mill.) B.L. Rob.

Crusea longiflora (Roem. \& Schult.) W.R. Anderson

*Crusea megalocarpa (A. Gray) S. Watson

Crusea parviflora Hook. \& Arn.

*Crusea psyllioides (Kunth) W.R. Anderson

Crusea setosa (M. Martens \& Galeotti) Standl. \& Steyerm.

Crusea wrightii A. Gray

*Deppea cornifolia (Benth.) Benth.

*Deppea hintonii Bullock

Didymaea alsinoides (Schltdl. \& Cham.) Standl.

* Didymaea floribunda Rzed.

*Edithea guerrerensis (Dwyer \& Lorence) Borhidi \& Strancz.

Exostema caribaeum (Jacq.) Schult.

Galium aschenbornii S. Schauer

Galium mexicanum Kunth

* Galium praetermissum Greenm.

*Galium seatonii Greenm.

Galium sphagnophilum (Greenm.) Dempster

Galium trifidum L.

Galium uncinulatum DC.

Guettarda elliptica Sw.

Hamelia patens Jacq.

*Hamelia versicolor A. Gray

*Hamelia xorullensis Kunth

Hedyotis wrightii (A. Gray) Fosberg

Hintonia latiflora (Sessé \& Moc. ex DC.) Bullock

*Hoffmannia cuneatissima B.L. Rob.

Margaritopsis microdon (DC.) C.M. Taylor

Mitracarpus hirtus (L.) DC. 
Apéndice 4: Continuación.

*Paederia ciliata (Bartl. ex DC.) Standl.

Randia aculeata $\mathrm{L}$.

Randia capitata DC.

*Randia cinerea (Fernald) Standl.

Randia echinocarpa Sessé \& Moc. ex DC.

Randia monantha Benth.

* Randia obcordata S. Watson

* Randia tetracantha (Cav.) DC.

* Randia thurberi $\mathrm{S}$. Watson

Relbunium hypocarpium (L.) Hemsl.

Relbunium laevigatum (DC.) Hemsl.

Richardia scabra L.

Richardia tricocca (Torr. \& A. Gray) Standl.

* Simira mexicana (Bullock) Steyerm.

Spermacoce confusa Rendle

Spermacoce ovalifolia (M. Martens \& Galeotti) Hemsl.

Spermacoce tenuior $\mathrm{L}$.

\section{Rutaceae}

*Amyris rekoi S.F. Blake

Casimiroa edulis La Llave \& Lex.

*Choisya ternata Kunth

Ptelea trifoliata L.

Zanthoxylum fagara (L.) Sarg.

Zanthoxylum limoncello Planch. \& Oerst.

\section{Sabiaceae}

Meliosma dentata (Liebm.) Urb.

\section{Salicaceae}

Casearia aculeata Jacq.

Casearia nitida (L.) Jacq.

Casearia tremula (Griseb.) Griseb. ex C. Wright

*Neopringlea integrifolia (Hemsl.) S. Watson

Neopringlea viscosa (Liebm.) Rose

*Populus simaroa Rzed.

Populus tremuloides Michx.

Prockia crucis P. Browne ex L.

Salix bonplandiana Kunth

* Salix cana M. Martens \& Galeotti

*Salix hartwegii Benth.

Salix humboldtiana Willd.

Salix lasiolepis Benth.

* Salix mexicana Seemen

* Salix paradoxa Kunth

Salix taxifolia Kunth
Xylosma flexuosa (Kunth) Hemsl.

Xylosma intermedia (Seem.) Triana \& Planch.

\section{Santalaceae}

*Arceuthobium abietis-religiosae Heil

Arceuthobium globosum Hawksw. \& Wiens

Arceuthobium vaginatum (Humb. \& Bonpl. ex Willd.) J. Presl

Phoradendron brachystachyum (DC.) Eichler

*Phoradendron carneum Urb.

Phoradendron falcatum Eichler

*Phoradendron galeottii Trel.

*Phoradendron lanceolatum Engelm. ex A. Gray

* Phoradendron longifolium Eichler ex Trel.

* Phoradendron perredactum Rzed. \& Calderón

Phoradendron piperoides (Kunth) Trel.

Phoradendron quadrangulare (Kunth) Griseb.

Phoradendron reichenbachianum (Seem.) Oliv.

* Phoradendron rhipsalinum Rzed.

Phoradendron robinsonii Urb.

Phoradendron velutinum (DC.) Eichler

\section{Sapindaceae}

Acer negundo L.

Cardiospermum grandiflorum Sw.

Cardiospermum halicacabum L.

Dodonaea viscosa (L.) Jacq.

Paullinia tomentosa Jacq.

Sapindus saponaria L.

Serjania cardiospermoides Schltdl. \& Cham.

Serjania mexicana (L.) Willd.

Serjania racemosa Schumach.

*Serjania schiedeana Schltdl.

* Serjania trifoliolata Radlk.

Serjania triquetra Radlk.

Thouinia paucidentata Radlk.

*Thouinia villosa DC.

Thouinidium decandrum (Bonpl.) Radlk.

Urvillea ulmacea Kunth

\section{Sapotaceae}

Manilkara zapota (L.) P. Royen

Pouteria campechiana (Kunth) Baehni

Pouteria sapota (Jacq.) H.E. Moore \& Stearn

Sideroxylon capiri (A. DC.) Pittier

* Sideroxylon palmeri (Rose) T.D. Penn.

Sideroxylon tepicense (Standl.) T.D. Penn. 
Apéndice 4: Continuación.

\section{Saxifragaceae}

*Heuchera mexicana W. Schaffn. ex Small \& Rydb.

*Heuchera orizabensis Hemsl.

\section{Schoepfiaceae}

Schoepfia flexuosa (Ruiz \& Pav.) Schult.

*Schoepfia pringlei B.L. Rob.

\section{Scrophulariaceae}

Buddleja americana L.

Buddleja cordata Kunth

*Buddleja parviflora Kunth

*Buddleja perfoliata Kunth

Buddleja scordioides Kunth

Buddleja sessiliflora Kunth

Capraria biflora L.

Capraria frutescens (Mill.) Britten

Limosella aquatica L.

\section{Solanaceae}

Bouchetia erecta DC. ex Dunal

Brachistus stramoniifolius (Kunth) Miers

Calibrachoa parviflora (Juss.) D'Arcy

Capsicum annuum $\mathrm{L}$.

Capsicum rhomboideum (Dunal) Kuntze

Cestrum aurantiacum Lindl.

* Cestrum fulvescens Fernald

Cestrum laxum Benth.

*Cestrum nitidum M. Martens \& Galeotti

Cestrum nocturnum $\mathrm{L}$.

*Cestrum oblongifolium Schltdl.

Cestrum roseum Kunth

Cestrum thyrsoideum Kunth

Cestrum tomentosum L. f.

* Datura ceratocaula Ortega

Datura inoxia Mill.

Datura quercifolia Kunth

Datura stramonium L.

* Jaltomata bohsiana Mione \& D.M. Spooner

Jaltomata procumbens (Cav.) J.L. Gentry

Lycianthes acapulcensis (Baill.) D'Arcy

Lycianthes arrazolensis (J.M. Coult. \& Donn. Sm.) Bitter

Lycianthes ciliolata (M. Martens \& Galeotti) Bitter

*Lycianthes dejecta (Fernald) Bitter

Lycianthes moziniana (Dunal) Bitter

*Lycianthes peduncularis (Schltdl.) Bitter
*Lycianthes pringlei (B.L. Rob. \& Greenm.) Bitter

*Lycianthes starbuckii E. Dean

*Lycianthes surotatensis Gentry

Lycianthes tricolor (Sessé \& Moc. ex Dunal) Bitter

Lycium carolinianum Walter

Nectouxia formosa Kunth

Nicotiana obtusifolia M. Martens \& Galeotti

* Nierembergia angustifolia Kunth

Physalis angulata L.

Physalis cinerascens (Dunal) Hitchc.

*Physalis coztomatl Moc. \& Sessé ex Dunal

Physalis gracilis Miers

*Physalis hintonii Waterf.

Physalis lagascae Roem. \& Schult.

Physalis microcarpa Urb. \& Ekman

Physalis mollis Nutt.

Physalis nicandroides Schltdl.

*Physalis orizabae Dunal

*Physalis patula Mill.

Physalis peruviana L.

Physalis philadelphica Lam.

*Physalis pringlei Greenm.

Physalis pruinosa L.

Physalis pubescens L.

Physalis solanacea (Schltdl.) Axelius

*Physalis sordida Fernald

*Physalis subrepens Waterf.

*Physalis sulphurea (Fernald) Waterf.

Physalis viscosa $\mathrm{L}$.

* Solandra guerrerensis Martínez

*Solandra guttata D. Don

Solandra nitida Zuccagni

Solanum aligerum Schltdl.

Solanum americanum Mill.

Solanum angustifolium Mill.

Solanum aphyodendron S. Knapp

Solanum appendiculatum Humb. \& Bonpl. ex Dunal

Solanum bulbocastanum Dunal

Solanum candidum Lindl.

*Solanum cardiophyllum Lindl.

Solanum chrysotrichum Schltdl.

Solanum corymbosum Jacq.

Solanum demissum Lindl.

Solanum diphyllum L.

Solanum donianum Walp.

Solanum douglasii Dunal 
Apéndice 4: Continuación.

Solanum dulcamaroides Dunal

*Solanum ehrenbergii (Bitter) Rydb.

Solanum elaeagnifolium Cav.

Solanum erianthum D. Don

*Solanum fructu-tecto Cav.

Solanum heterodoxum Dunal

* Solanum hintonii Correll

Solanum hispidum Pers.

*Solanum iopetalum (Bitter) Hawkes

Solanum laurifolium Mill.

Solanum mitlense Dunal

Solanum morelliforme Bitter \& Münch

Solanum nigrescens $\mathrm{M}$. Martens \& Galeotti

Solanum nigricans $\mathrm{M}$. Martens \& Galeotti

Solanum nudum Dunal

*Solanum pedunculare Schltdl.

*Solanum polyadenium Greenm.

Solanum pubigerum Dunal

Solanum rostratum Dunal

Solanum rudepannum Dunal

*Solanum stenophyllidium Bitter

Solanum stoloniferum Schltdl. \& C.D. Bouché

Solanum torvum Sw.

Solanum umbellatum Mill.

*Solanum verrucosum Schltdl.

\section{Stegnospermataceae}

Stegnosperma cubense A. Rich.

Stegnosperma scandens (Lunan) Standl.

\section{Styracaceae}

Styrax argenteus $\mathrm{C}$. Presl

Styrax glabrescens Benth.

*Styrax ramirezii Greenm.

\section{Symplocaceae}

*Symplocos citrea Lex.

\section{Talinaceae}

Talinum fruticosum (L.) Juss.

Talinum lineare Kunth

Talinum paniculatum (Jacq.) Gaertn.

\section{Urticaceae}

Boehmeria ulmifolia Wedd.

Cecropia obtusifolia Bertol.
Discocnide mexicana (Liebm.) Chew

* Myriocarpa brachystachys S. Watson

Parietaria debilis G. Forst.

*Parietaria macrophylla B.L. Rob. \& Greenm.

Parietaria pensylvanica Muhl. ex Willd.

Phenax hirtus (Sw.) Wedd.

Pilea microphylla (L.) Liebm.

Pouzolzia guatemalana (Blume) Wedd.

Pouzolzia occidentalis (Liebm.) Wedd.

Urera baccifera (L.) Gaudich. ex Wedd.

Urera verrucosa (Liebm.) V.W. Steinm.

Urtica chamaedryoides Pursh

Urtica dioica $\mathrm{L}$.

Urtica mexicana Liebm.

*Urtica subincisa Benth.

\section{Verbenaceae}

Bouchea prismatica (L.) Kuntze

Citharexylum affine D. Don

*Citharexylum glabrum (S. Watson) Greenm.

*Citharexylum hintonii Moldenke

Duranta erecta L.

* Glandularia amoena (Paxton) Umber

Glandularia bipinnatifida (Nutt.) Nutt.

Glandularia elegans (Kunth) Umber

Glandularia teucriifolia (M. Martens \& Galeotti) Umber

Lantana achyranthifolia Desf.

Lantana camara L.

Lantana glandulosissima Hayek

*Lantana langlassei Moldenke

Lantana trifolia $\mathrm{L}$.

Lippia alba (Mill.) N.E. Br. ex Britton \& P. Wilson

Lippia graveolens Kunth

*Lippia mexicana G.L. Nesom

Lippia myriocephala Schltdl. \& Cham.

Lippia umbellata Cav.

Petrea volubilis $\mathrm{L}$.

Phyla dulcis (Trevir.) Moldenke

Phyla nodiflora (L.) Greene

Priva aspera Kunth

* Priva grandiflora (Ortega) Moldenke

Priva lappulacea (L.) Pers.

Priva mexicana (L.) Pers.

*Stachytarpheta hintonii Moldenke

* Stachytarpheta mexicana Moldenke

Stachytarpheta mutabilis (Jacq.) Vahl 
Apéndice 4: Continuación.

*Stachytarpheta velutina Moldenke

Verbena canescens Kunth

Verbena carolina $\mathrm{L}$.

Verbena gracilis Desf.

Verbena litoralis Kunth

Verbena menthifolia Benth.

*Verbena recta Kunth

\section{Violaceae}

Hybanthus attenuatus (Humb. \& Bonpl. ex Roem. \& Schult.) Schulze-Menz

Hybanthus verbenaceus (Kunth) Loes.

Hybanthus verticillatus (Ortega) Baill.

Ixchelia mexicana (Ging.) H.E. Ballard \& Wahlert

*Viola beamanii Calderón

Viola grahamii Benth.

Viola guatemalensis W. Becker

*Viola hemsleyana Calderón

*Viola hookeriana Kunth

Viola humilis Kunth

*Viola painteri Rose \& House

Viola umbraticola Kunth

\section{Vitaceae}

Ampelocissus acapulcensis (Kunth) Planch.

Ampelopsis denudata Planch.

*Cissus cucurbitina Standl.

Cissus microcarpa Vahl

*Cissus subtruncata Rose

Cissus tiliacea Kunth

Cissus trifoliata (L.) L.

Cissus verticillata (L.) Nicolson \& C.E. Jarvis

Vitis bourgaeana Planch.

Vitis cinerea (Engelm.) Engelm. ex Millardet

Vitis tiliifolia Humb. \& Bonpl. ex Roem. \& Schult.

\section{Zygophyllaceae}

Kallstroemia maxima (L.) Hook. \& Arn.

Kallstroemia parviflora Norton

*Kallstroemia rosei Rydb. 